\title{
SUMMARY OF KEY DIRECTIVES GOVERNING PERMANENT DISPOSAL IN A GEOLOGIC REPOSITORY
}

\author{
S. C. Sands III
}

November 1993

Westinghouse Idaho

PREPARED FOR THE DEPARTMENT OF ENERGY IDAHO OPERATIONS OFFICE UNDER CONTRACT DE-AC07-84ID12435 
EXECUTIVE SUMMARY $\ldots \ldots \ldots \ldots \ldots \ldots \ldots$ ii

1.0 General Introduction $\ldots \ldots \ldots \ldots \ldots \ldots \ldots \ldots \ldots \ldots$

1.1 Legislative History $\ldots \ldots \ldots \ldots \ldots \ldots \ldots \ldots \ldots$

1.2 Regulation Development . . . . . . . . . . . . . . . 4

2.0 Transportation Requirements . . . . . . . . . . . . . . . 7

2.1 Listing of Regulations and Orders Developed ......... 7

2.2 Flow Diagrams for Regulations and Orders $\ldots \ldots \ldots \ldots \ldots$

3.0 Mined Geologic Disposal System (MGDS) Requirements . . . . . . . . 26

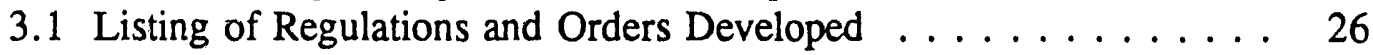

3.2 Flow Diagrams for Regulations and Orders . . . . . . . . 27

ACRONYMS $\ldots \ldots \ldots \ldots \ldots \ldots \ldots \ldots \ldots \ldots \ldots \ldots \ldots$

INDEX OF DIRECTIVES $\ldots \ldots \ldots \ldots \ldots \ldots \ldots \ldots \ldots$

REFERENCES . . . . . . . . . . . . . . . . . . . . . . 49

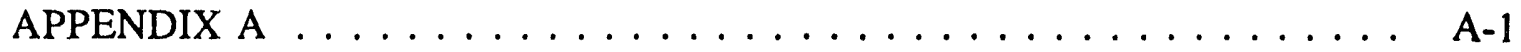




\section{EXECUTIVE SUMMARY}

This document was developed in support of the Idaho National Engineering Laboratory (INEL) Spent Fuel and Waste Management Technology Development Program (SF\&WMTDP). It is largely comprised of flow diagrams summarizing the key regulatory requirements which govern permanent disposal in a geologic repository. The key purposes are (1) to provide an easy and effective tool for referencing or cross referencing federal directives (i.e., regulations and orders), (2) to provide a method for examining the requirements in one directive category against the requirements in another, and (3) to list actions that must be taken to ensure directive compliance. The document is categorically broken down into a Transportation section and a Mined Geologic Disposal System (MGDS) section to ensure that the interrelationship of the entire disposal system is considered.

The Transportation section describes the transportation packaging requirements, testing methods, and safety requirements imposed on fissile material shipments.

The MGDS section encompasses technical aspects involved in siting, licensing, waste interaction with the container, container design features, physical characteristics of the surrounding environment, facility design features, barrier systems, safety features, criticality considerations, migration restrictions, implementation guidelines, and so forth. Whether the hazardous waste requirements of 40 CFR 260-270 will apply to geologic disposal of some materials is not clear at this time; as this issue is developed, this summary will be revised accordingly. For purposes of illustration, the worst case scenario (i.e., that a permit and a variance will be required for disposal) is outlined (a discussion of the permitting process is not included).

It is important that the approaches and considerations contained in this document be integrated into the efforts of the SF\&WMTDP so that every applicable aspect of the regulatory requirements can be evaluated to avoid investing large sums of money into projects that do not take into account all of the aspects of permanent waste disposal. Not until an overall picture and clear understanding of these regulations is established can a basis be developed to govern the direction of future activities of the SF\&WMTDP. 


\subsection{General Intre ${ }^{-1}$ iction}

This document summarizes federal directives impacting disposal at a geologic repository. The intention here is three-fold: (1) to provide a tool for referencing current directive requirements, (2) to provide a method for examining the requirements in one directive category against the requirements in another, and (3) to list actions that must be taken to ensure directive compliance. This document will require periodic reviews and updates to reflect changes in applicable directives. The directives discussed herein have been divided into Transportation Requirements and Mined Geologic Disposal System (MGDS) Requirements. Figure 1 provides a visual summary of the requirements necessary for the permanent disposal in a geologic repository.

Flow diagrams, comprising the bulk portion of this document, have been prepared on various levels of detail; the objective is to provide sufficient detail in the flow diagrams so that they can stand alone from the sources of the requirements in most instances. For directives containing large amounts of detailed information, several flow diagrams have been prepared. These descriptions include a summary sheet outlining the information found in the particular regulation or DOE Order. Whenever possible, detailed flow diagrams are developed for all of the subparts contained in each directive.

Each flow sheet is organized into a series of columns: Compliance Approach (listing overall methods for assessing compliance with a particular regulatory requirement); Compliance Support (listing the specific items or topics addressed in that regulation); and Considerations, Information Needs, and Activities (summarizing the significant details covered by that specific regulation or section). Suppose, for example, it is necessary to find applicable directives pertaining to transportation. The Compliance Approach and Support columns would be scanned for transportation related issues resulting in a listing of specific information and directives applicable to the transportation.

It is important that these approaches and considerations be integrated into the activities of the SF\&WMTDP so that every applicable requirement can be evaluated to avoid investing large sums of money into projects that do not take into account the long term goal of permanent waste disposal. Not until an overall picture of the applicable directives is established can a basis be developed to govern the direction of future activities of the SF\&WMTDP. 
Figure 1

\section{Pictorial Summary of}

\section{Requirements Developed}

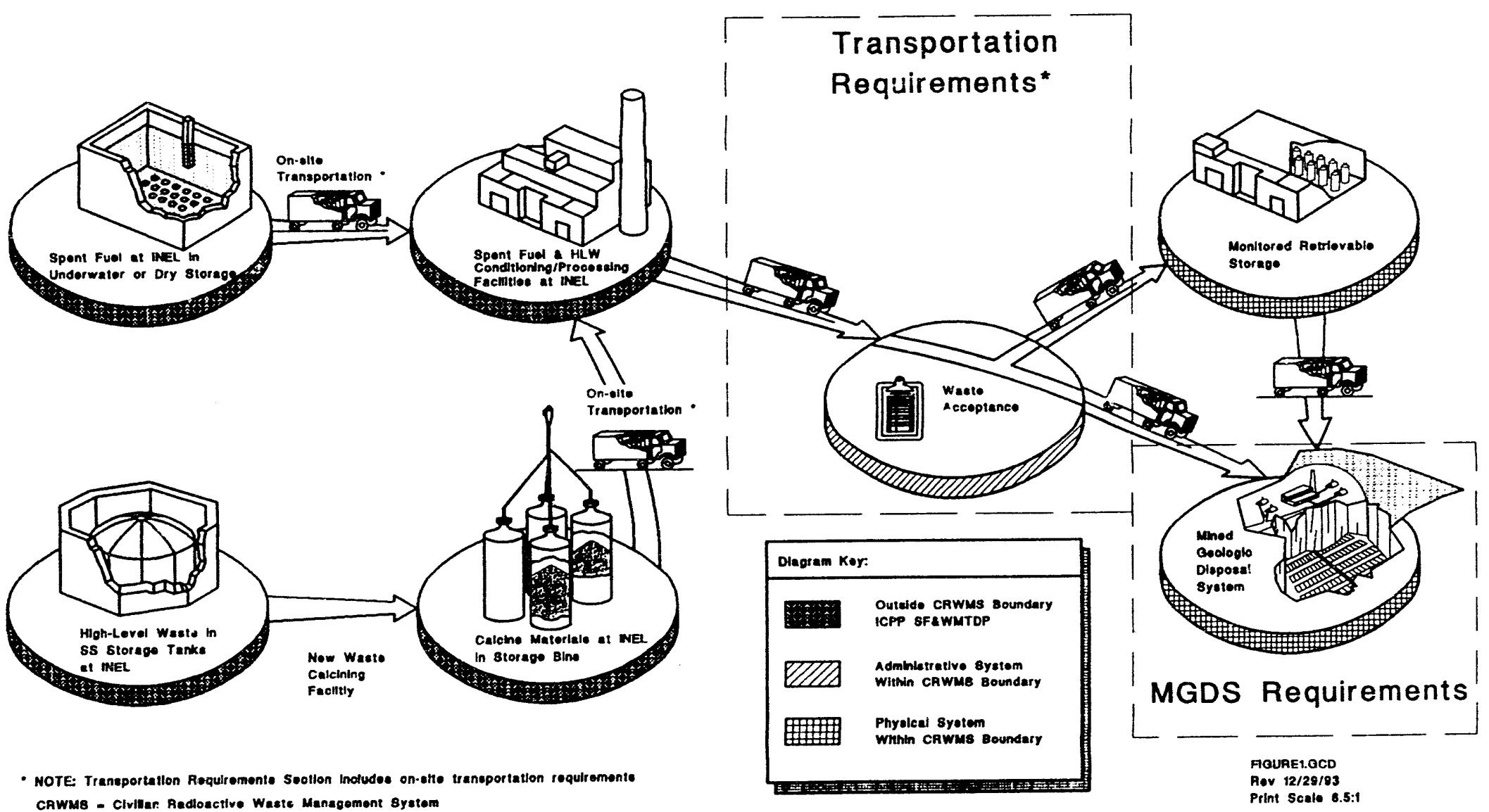




\subsection{Congressional Actions Impacting Geologic Disposal}

In 1954, Congress passed the Atomic Energy Act (AEA) to assure proper management of source, special nuclear, and byproduct material. Section 161 of AEA provided the authority to establish "by rule, regulation, or order, such standards and instruction to govern the possession and use of special nuclear material, source material, and byproduct material as the Atomic Energy Commission may deem necessary or desirable to promote the common defense and security or to minimize danger to life or property." As a result, subsequent Congressional actions have been taken to address the long-term disposal of high-level radioactive waste, resulting in the establishment of such rules, regulations, and orders necessary to ensure compliance with federal legislation. Actions dealing with high-level waste (HLW) disposal include:

Energy Reorganization Act of 1974;

Nuclear Waste Policy Act (NWPA) of 1982;

Resource Conservation and Recovery Act (RCRA) of 1976 and the Hazardous and Solid Waste Amendments, which include Land Disposal Restriction (LDR), of 1984;

Nuclear Waste Policy Amendments Act (NWPAA) of 1987;

Federal Facilities Compliance Act (FFCA) of 1992; and

Energy Policy Act of 1992.

\subsection{Key Provisions of Applicable HLW Laws}

Energy Reorganization Act

Nuclear Regulatory Commission (NRC) licenses DOE HLW disposal.

\section{Nuclear Waste Policy Act and Amendments}

Yucca Mountain site is the only one to be characterized to determine suitability.

Capacity of first repository limited to 70,000 metric tonnes of heavy metal for spent fuel and equivalent DOE HLW.

Monitored Retrievable Storage (MRS) Facility cannot be constructed until after the NRC has issued a license for the repository. 
Congress to evaluate after 2007 of the need for a second repository.

NRC 10 CFR 60 to establish the licensing requirements for the repository.

EPA 40 CFR 191 to establish the standard for HLW and spent fuel disposal.

NRC Regulation 10 CFR 60

Waste retrievability during ( eration and 50 yrs after repository closure.

Pre-emplacement groundwater travel time of $>1000$ yrs to accessible environment.

Substantially complete containment of HLW in waste package for $300-1000$ yrs.

Limited release rate of radionuclides from waste package after 1000 yrs not to exceed 1 part in 100,000 per year of inventory at 1000 yrs.

Other waste package criteria to avoid in-situ chemical, physical or nuclear interactions which could compromise the performance of the repository.

EPA Standard 40 CFR 191 (Under re-evaluation for HLW by NAS)

Disposal system shall not cause annual dose equivalent to any person in the accessible environment to exceed $25 \mathrm{mrem}$ whole body or $75 \mathrm{mrem}$ critical organ.

Release of radionuclides to accessible environment over $10,000 \mathrm{yrs}$ limited to prescribed values given in paragraph 13 of standard.

Additional requirements to provide additional assurance that standard will be met including institutional controls, monitoring, permanent markers and records, engineered and natural barriers, and avoidance of areas with mined resources.

\subsection{Regulation Development}

Under the NWPA, a process for the selection of sites for HLW repositories is established, and the DOE is charged with the responsibility of locating, constructing, operating, closing, and decommissioning repositories. This Act also stipulates that the disposal of waste be governed by an environmental standard established by the EPA. Responsibility for assuring that the DOE repository meets this EPA established standard is delegated to the NRC.

In 1983, the NRC developed a procedure for assuring that future repository 
investigations and developments are in compliance with the requirements of the NWPA. This procedure was documented under Title 10 of the Code of Federal Regulations, Chapter 60 (10 CFR 60). This regulation currently requires that the standard established in 40 CFR 191 must be met and, in addition, sets numerical requirements for the performance of some repository elements. In the license application, DOE must illustrate that the repository will comply with both of the above regulations.

Because of the requirements placed on the DOE by 10 CFR 60, DOE established a regulation to provide general guidelines for the recommendation of sites for nuclear waste repositories. This regulation was documented in 1984 under Title 10 of the Code of Federal Regulations, Chapter 960. This regulation established procedural guidelines for implementing the requirements set forth in 10 CFR 60, including siting evaluation, nomination, and characterization; filing recommendations for repository development; and preclosure and postclosure guidelines.

The Department of Transportation (DOT) has also established regulations imposed on the DOE pertaining to the safe transportation of radioactive material. As a practical matter, these regulations become valid compliance issues influencing the development of the repository investigations required by the NWPA. Regulations stipulating that a package meeting repository requirements will automatically satisfy applicable transportation requirements do not exist; therefore, a repository packaging must be evaluated against both sets of regulations.

The EPA standard referred to above was promulgated in 1985 under Title 40 of the Code of Federal Regulations, Chapter 191 (40 CFR 191). This standard requires that any repository must protect the public from significant radiation doses for at least 10,000 years after permanent closure by releasing less than a predetermined amount of each radionuclide to the biosphere.

The regulatory requirements relating to the permanent disposal of HLW in a geologic repository are illustrated in Figure 2 and are listed according to the agency which imposes those regulations. 
Figure 2

\section{Regulations Affecting Repository Development by Imposing Agency =}

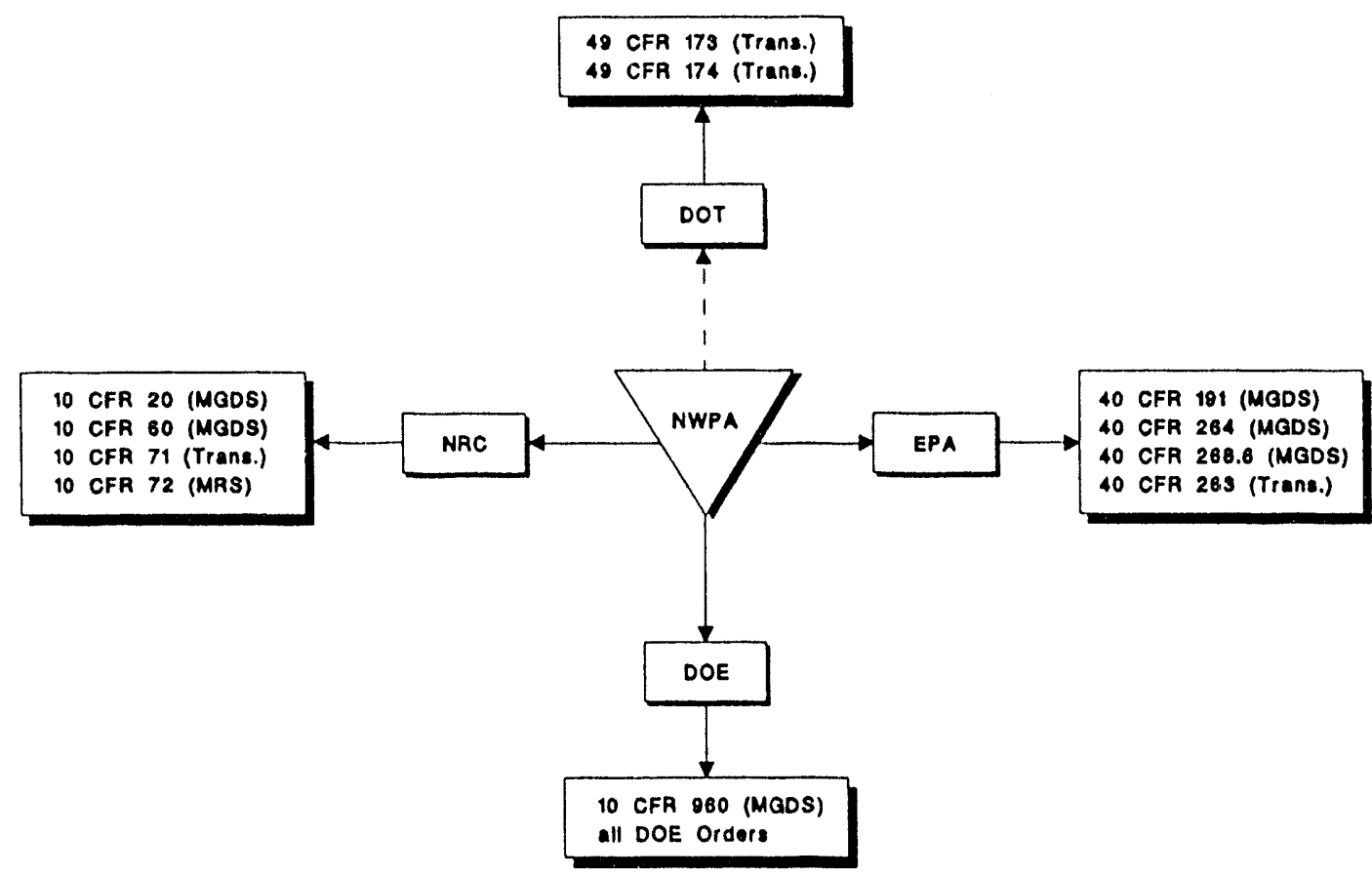

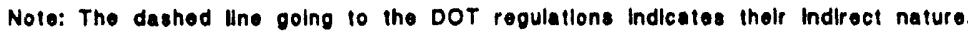

In an effort to assure compliance with these, and any other requirements (including requirements existing prior to the NWPA) having applicability to the permanent geologic disposal of HLW, the DOE imposes specific requirements and procedures in the form of DOE Orders. Applicable DOE Orders have been explained in the flow diagrams contained in their respective sections of this document.

In addition to Regulations and Orders, various guidance exists outlining specific procedures and practices for ensuring compliance with requirements of these directives. These guidance consist of notices, guidelines, memoranda, internal procedures, and implementation procedures. Because this document is a summary, only applicable Regulations and Orders have been summarized in the flow diagrams.

However, a brief listing of some potentially applicable Regulatory Guides is included in Appendix A. The words "potentially applicable" are used here because no detailed investigation into the applicability of these guides has been conducted. 


\subsection{Transportation Requirements}

Although it is the responsibility of the individual shipper to verify compliance with packaging and shipping regulations and to ensure that waste disposal canisters meet MGDS requirements, understanding these requirements will help ensure that the waste canisters that we produce or purchase are in compliance with applicable regulations. Section 2 focuses on analyzing the packaging requirements, package testing methods, and safety requirements imposed on fissile material shipments.

The type of package used is dependent on the activity of the radioactive material being considered. Type A packages cannot exceed A1 or A2 activity limits and are subject only to normal conditions of transport testing. Type B packages may exceed $\mathrm{A} 1$ or $\mathrm{A} 2$ activity limits and, therefore, require additional hypothetical accident conditions of transport testing. A table of these activity limits for single radionuclides and methods for calculating these activity limits for radionuclides not listed in the table (or for radionuclide mixtures) are contained in subsections 173.435 and 173.433, respectively, of Title 49 of the Code of Federal Regulations, subpart I of Part 173. According to the INEL Roadmap document, SNF will always contain fissile material in ex ress of these activity limits, thus requiring Type B packaging.

\subsection{Listing of Regulations and Orders Developed}

10 CFR 71 - Packaging and Transportation of Radioactive Material Subpart E - Package Approval Standards Subpart F - Package and Special Form Tests

49 CFR 173 - Shippers - General Requirements for Shipments and Packages (flow diagram listing sections developed) Subpart I - Radioactive Materials (Summary) Sections: - Type A or B Package Classification Method

- Cask/Canister Requirements \& Material Specific Restrictions

- Limitations and Transportation Requirements

- Fissile Material Requirements

- Testing Requirements

49 CFR 174 - Carriage by Rail Subpart K - Detailed Requirements for Radioactive Materials 40 CFR 263 - Standards Applicable to Transporters of Hazardous Materials DOE Order 1540.1A - Materials Transportation and Traffic Management 


$$
\begin{array}{cc}
\text { DOE Order 1540.2 } & \begin{array}{c}
\text { - Hazardous Materials Packaging for Transportation - } \\
\text { Administrative Procedures }
\end{array} \\
\text { DOE Order 5480.3 } & \begin{array}{c}
\text { - Safety Requirements for Packaging and Transportation } \\
\text { of Hazardous Materials, Substance, or Waste }
\end{array} \\
\text { DOE-ID 5480.3 } & \begin{array}{c}
\text { - Hazardous Materials Packaging and Transportation } \\
\text { Safety Requirements } \\
\text { Attachment 1 - Chapter I - Off-site Shipments } \\
\text { Attachment 1 - Chapter II - On-site Shipments }
\end{array}
\end{array}
$$

\subsection{Flow Diagrams for Regulations and Orders}

Flow diagrams are in the order listed in section 2.1. 
10 CFR 71 Packaging and Transportation of Radioactive Material

GOVERNING

REGULATION

Subparts

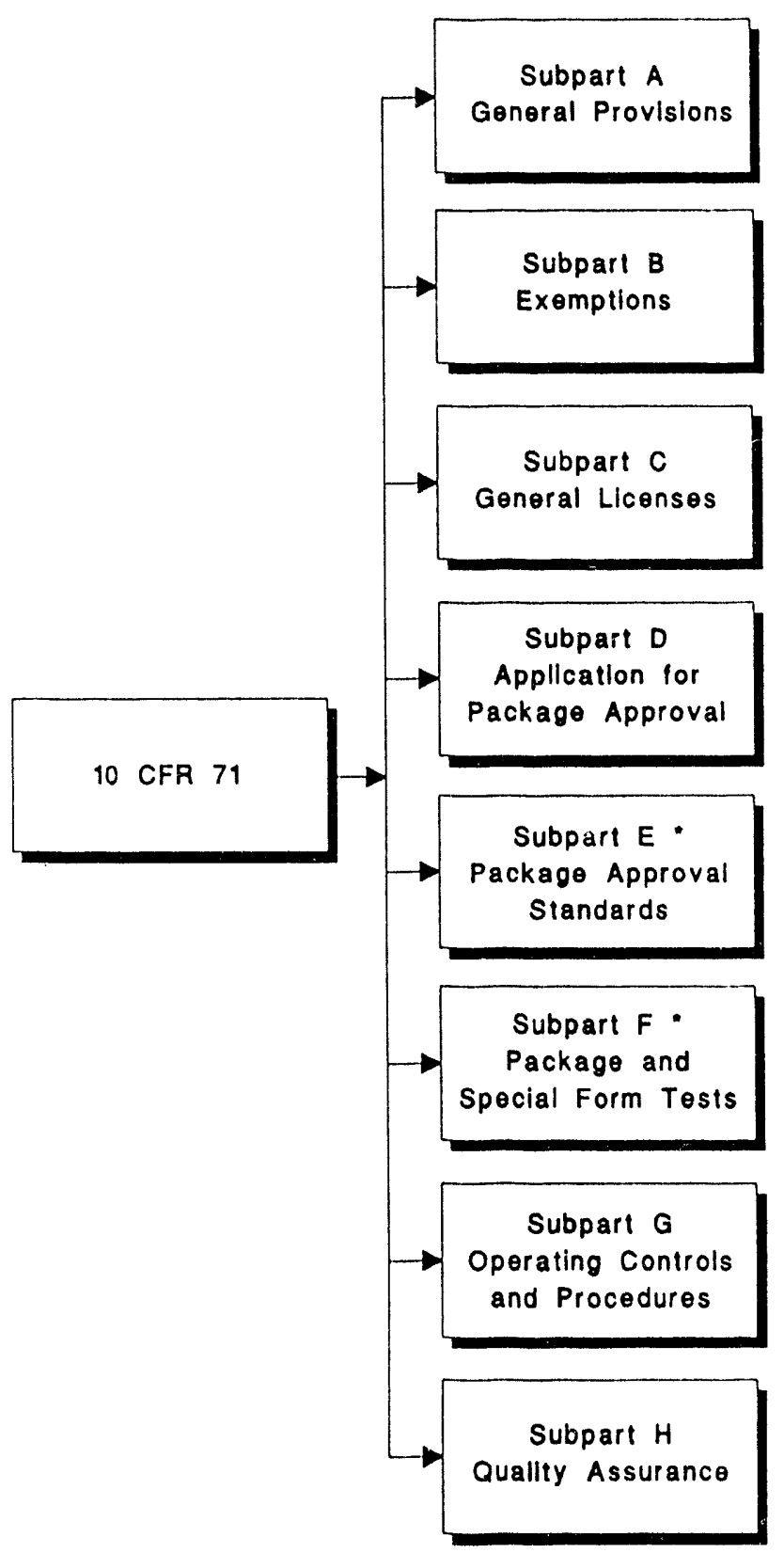

- Indleates that a flow dlagram for thle subpart hes been doveloped. 


\section{CFR 71 Subpart E Package Approval Standards}

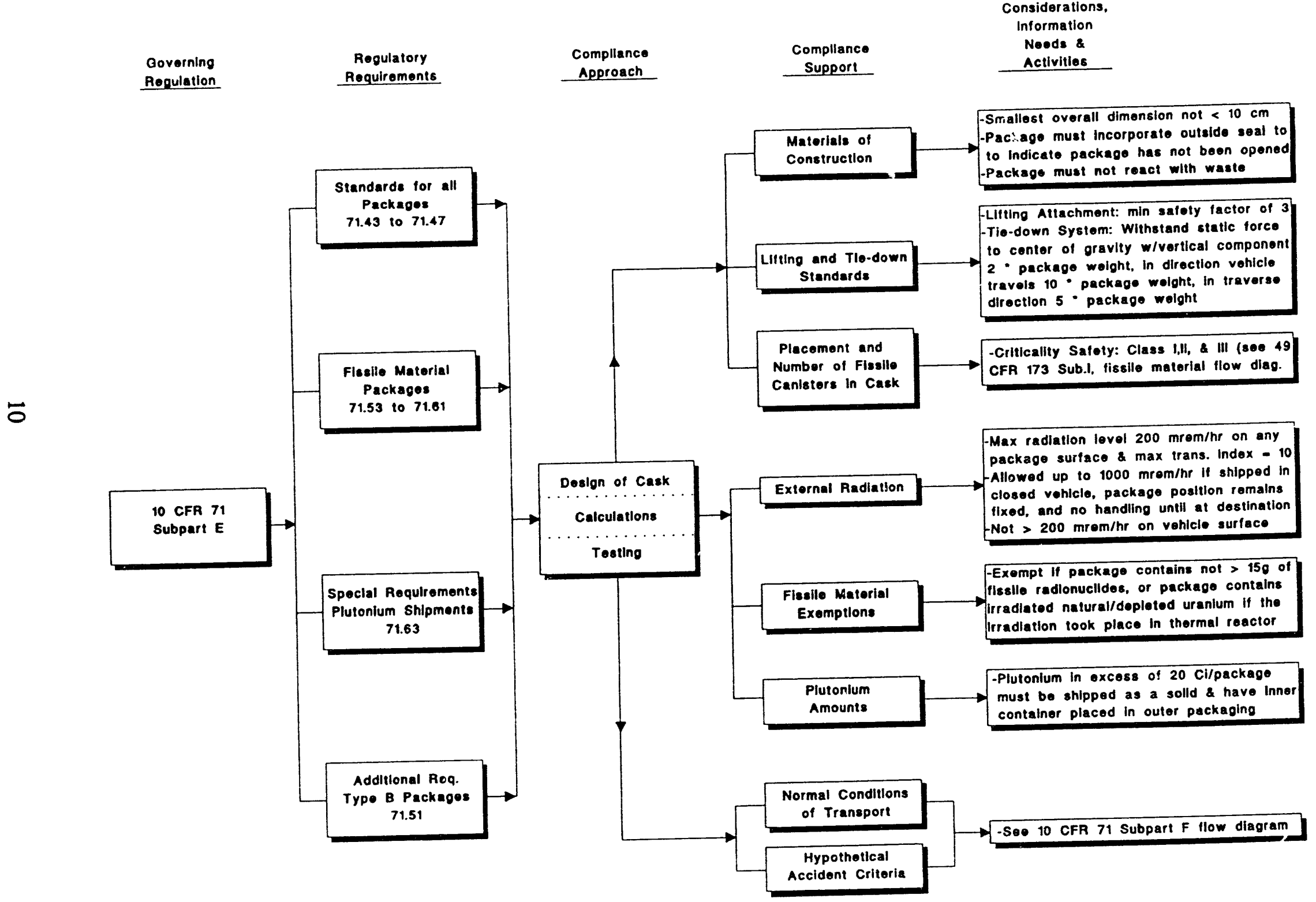




\section{CFR 71 Subpart F \\ Package and Special Form Tests \\ Rogulatory \\ Compllance \\ Requirements \\ Support \\ Considerations, \\ Information \\ Needs a \\ Activitios}

Regulation
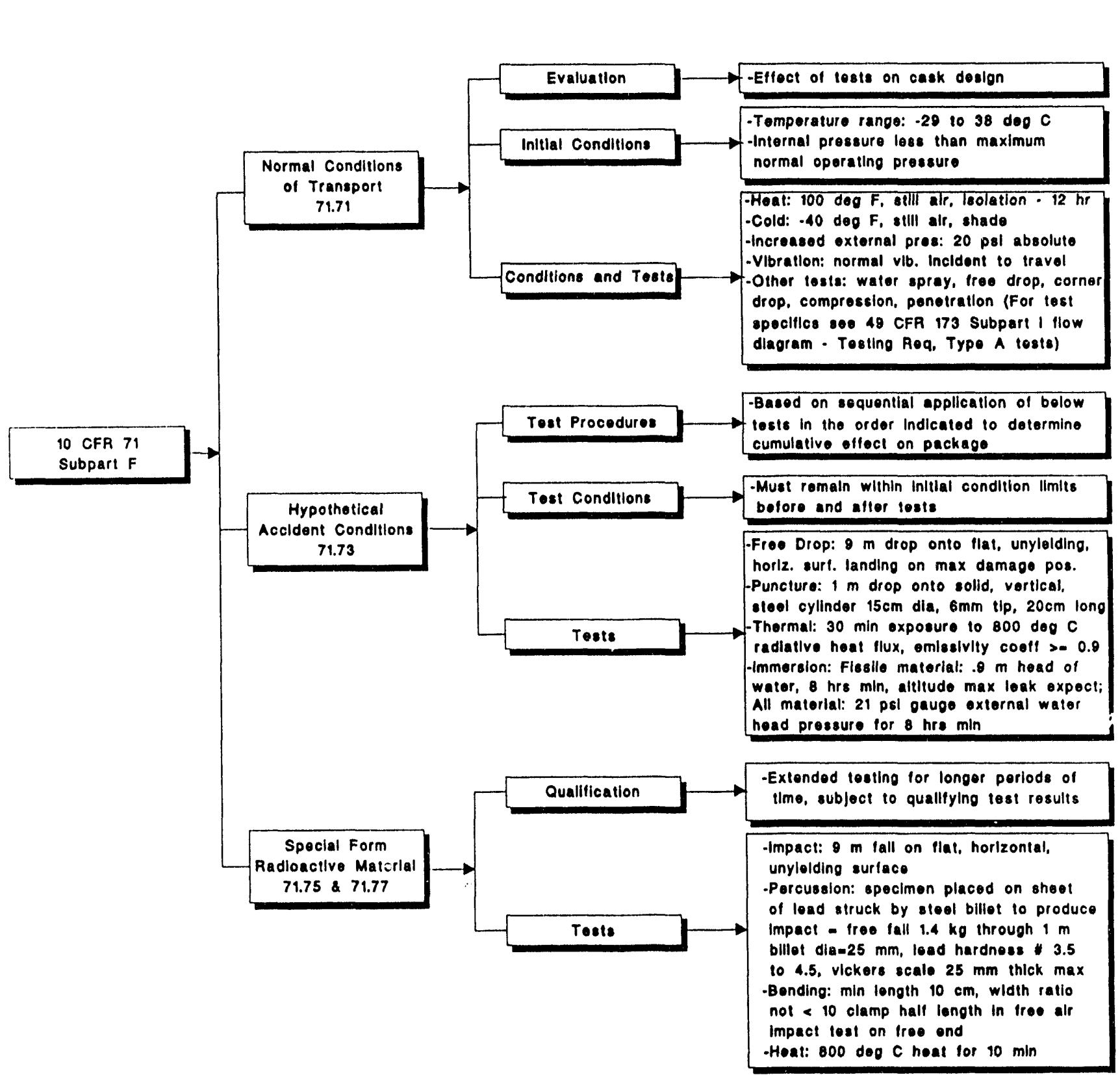
49 CFR 173 Shippers - General Requirements for Shipments and Packages

Subpart I - Radioactive Materials

Flow Diagram Listing

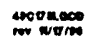

GOVERNINO

REGULATION

Flow Diagrams

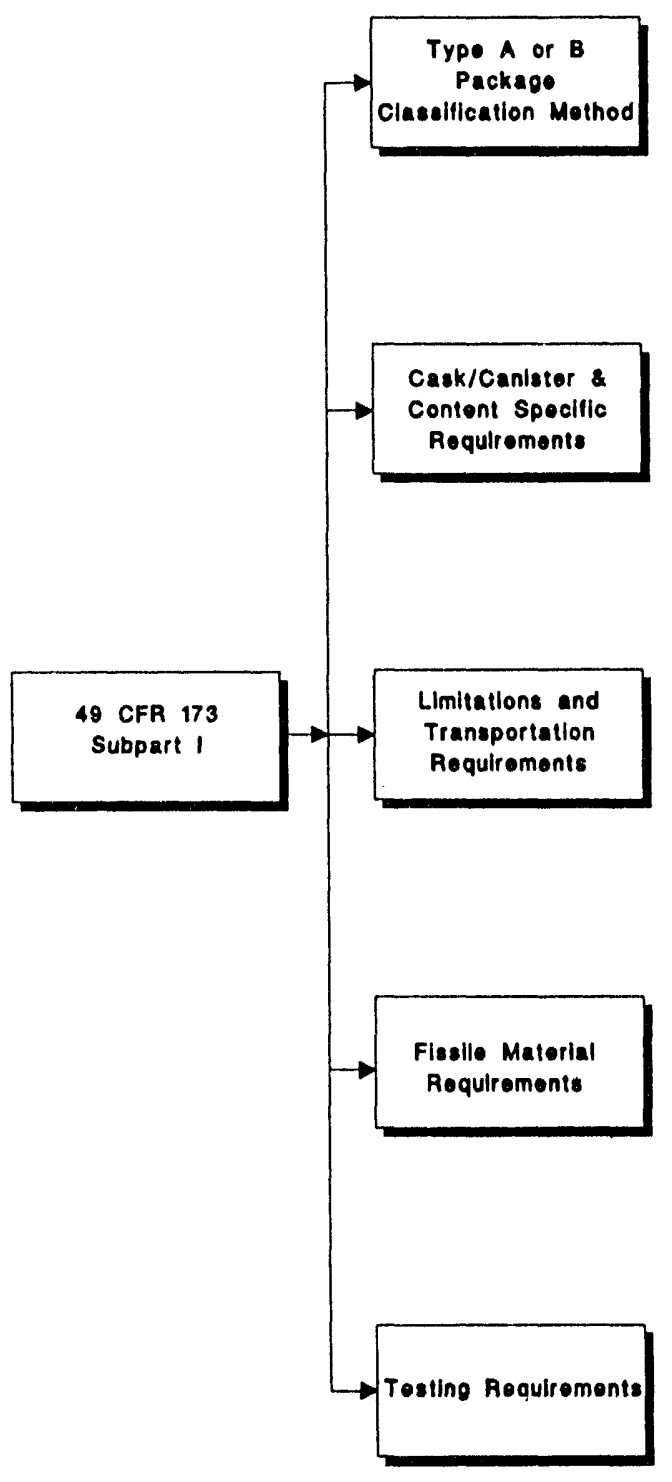




\section{CFR 173 Shippers - General Requirements for Shipments and Packages Subpart I - Radioactive Materials}

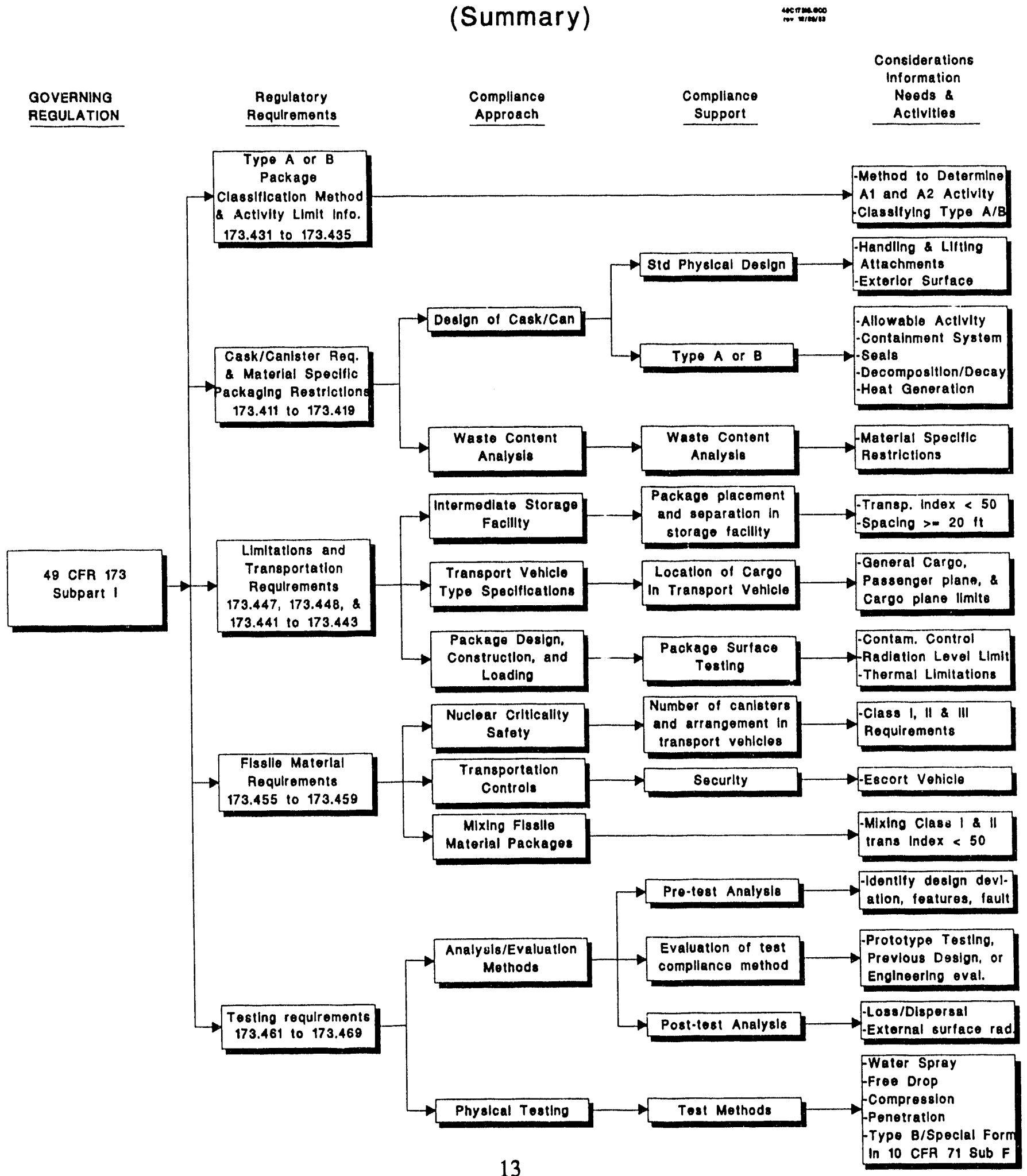




\section{CFR 173 Shippers - General Requirements for Shipments and Packages}

Subpart I - Radioactive Materials

Type A or B Package Classification Method

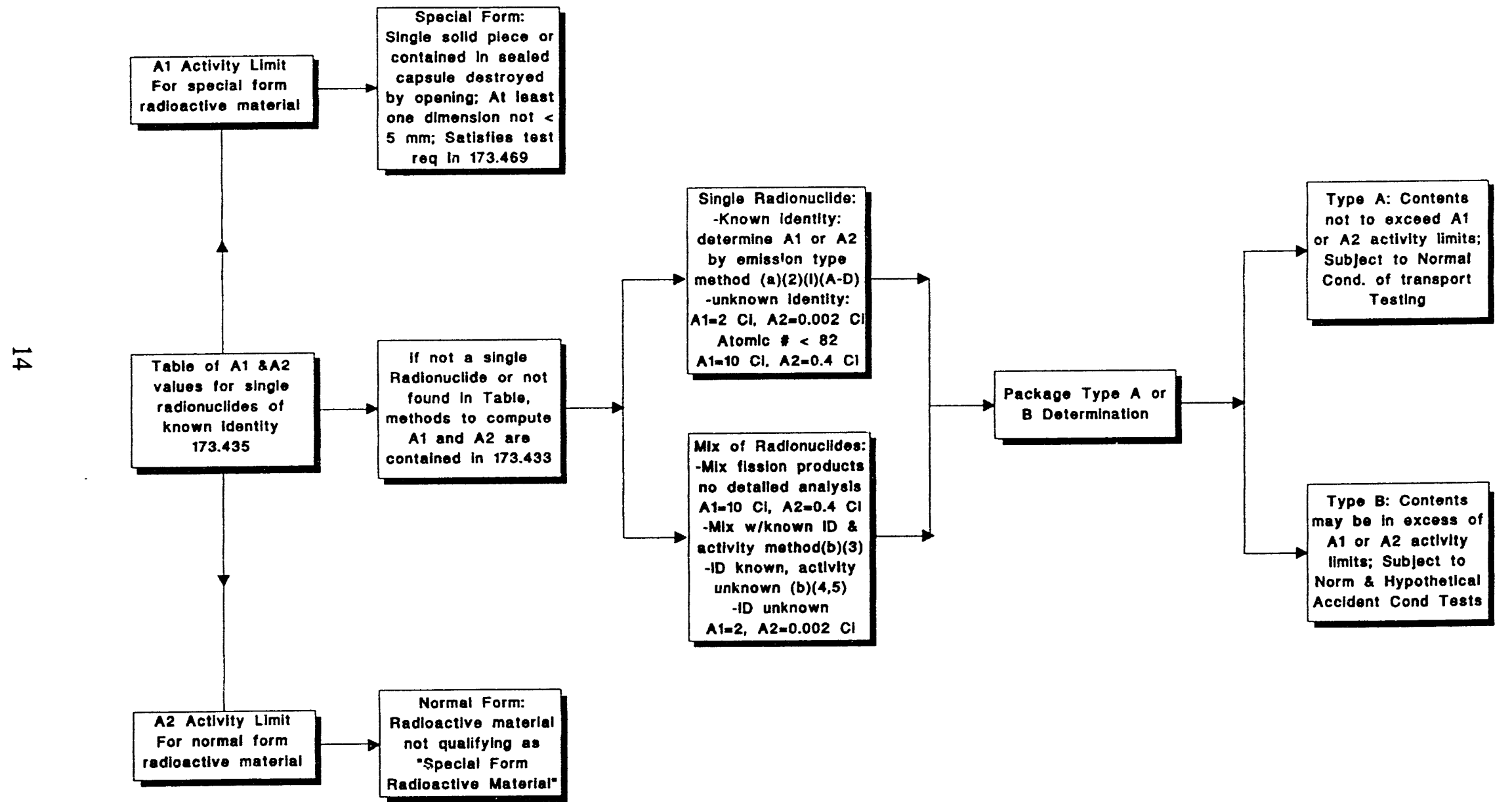

NOTE: SNF will always be a greater than Type A quantity and contain flssile materlals, thus requiring Type B shipping container. 


\section{Cask/Canister \& Content Specific Requirements}

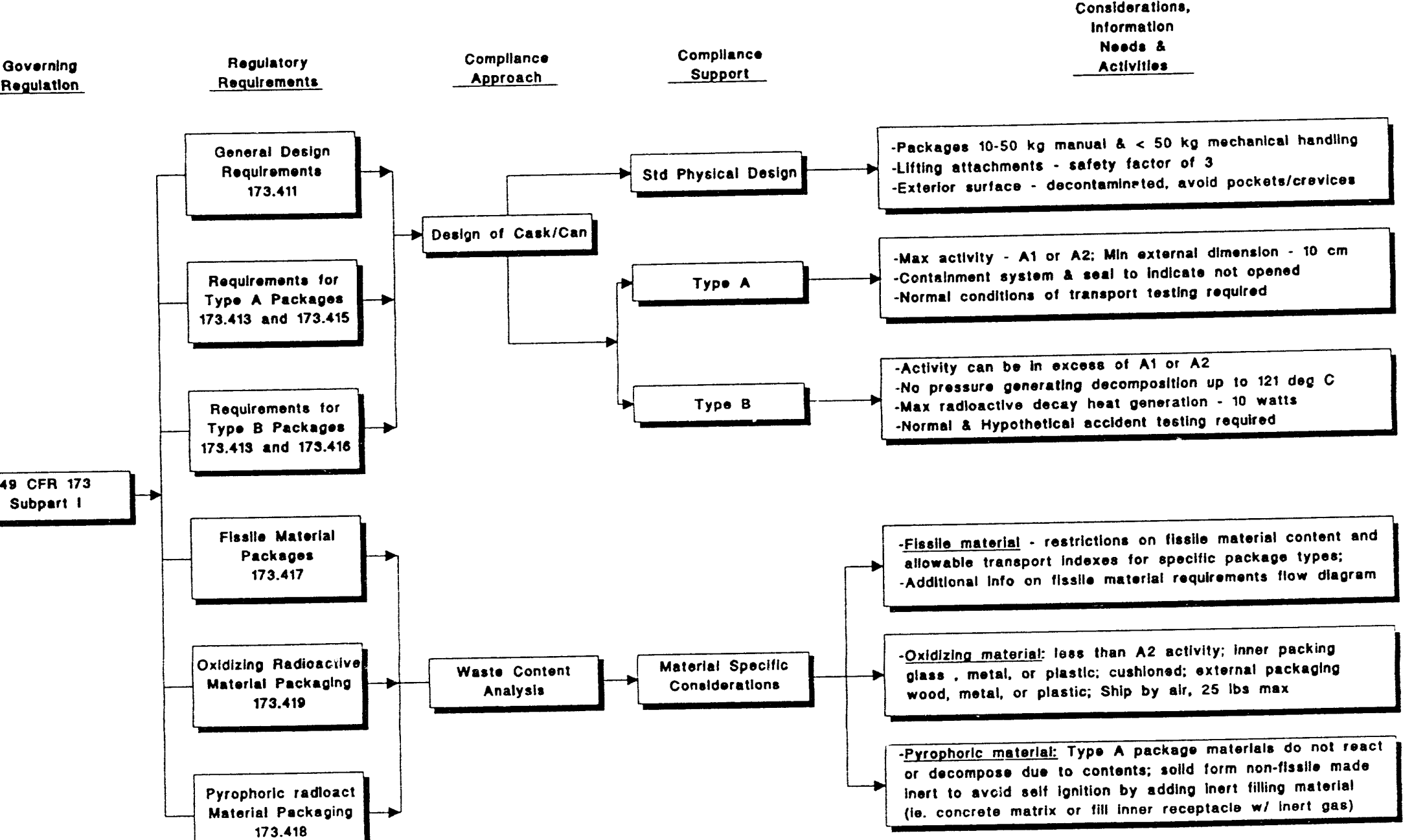




\section{CFR 173 Subpart I \\ Limitations and Transportation Requirements \\ Regulatory \\ Requirements \\ Compliance \\ Approach \\ Compliance \\ Support \\ Considerations. \\ Information \\ Neede \&}

Governing

Bogulation

mediate storago

During Transportation

173.447

Intermediate Storago

$\int$ Package placoment

and separation in

- Yellow II or III label: Sum of transport

$\longrightarrow$ Facilny

storage facility

any other radioactive materials

$\approx$

49 CFR 173

Subpart 1

Limitations

173.441 to 173.443
General Transport Requirements 173.448

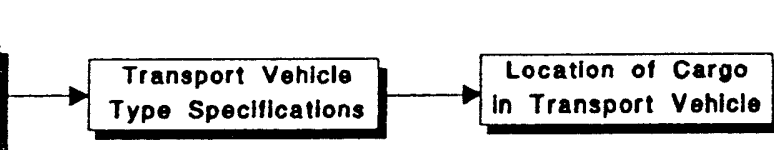

Package Design. Construction, and Loading Average surface heat flux $15 \mathrm{~W} / \mathrm{sq} \mathrm{m}$ Max transport index: 3.0 passenger plane, 10.0 cargo plane

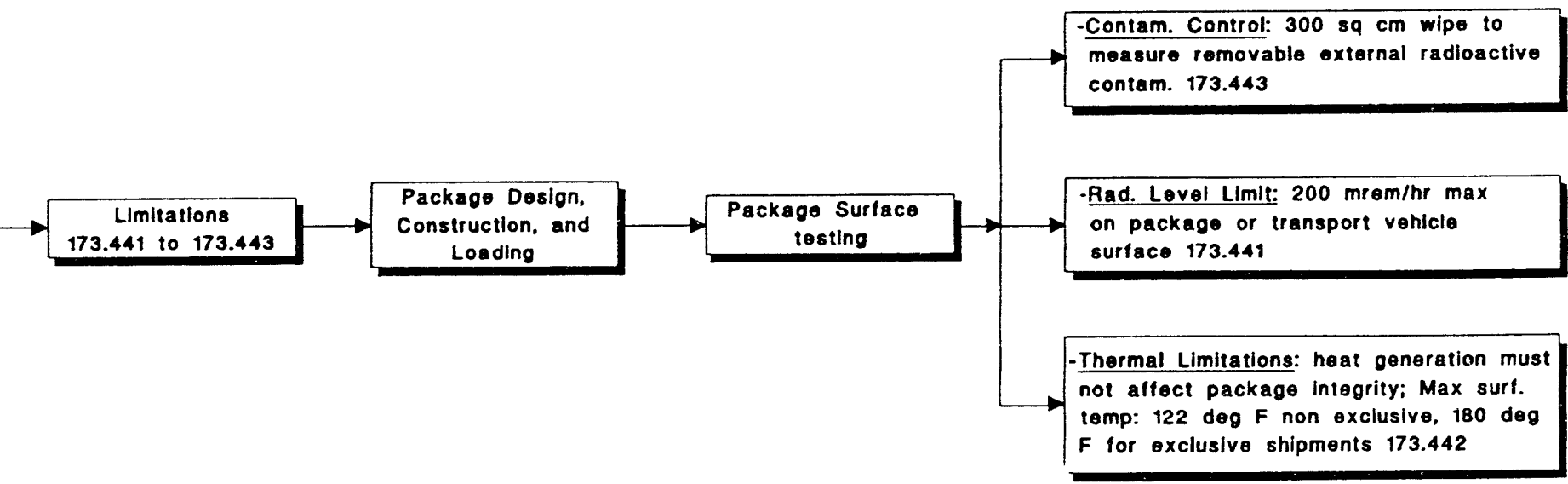




\section{CFR 173 Subpart I \\ Fissile Material Requirements}

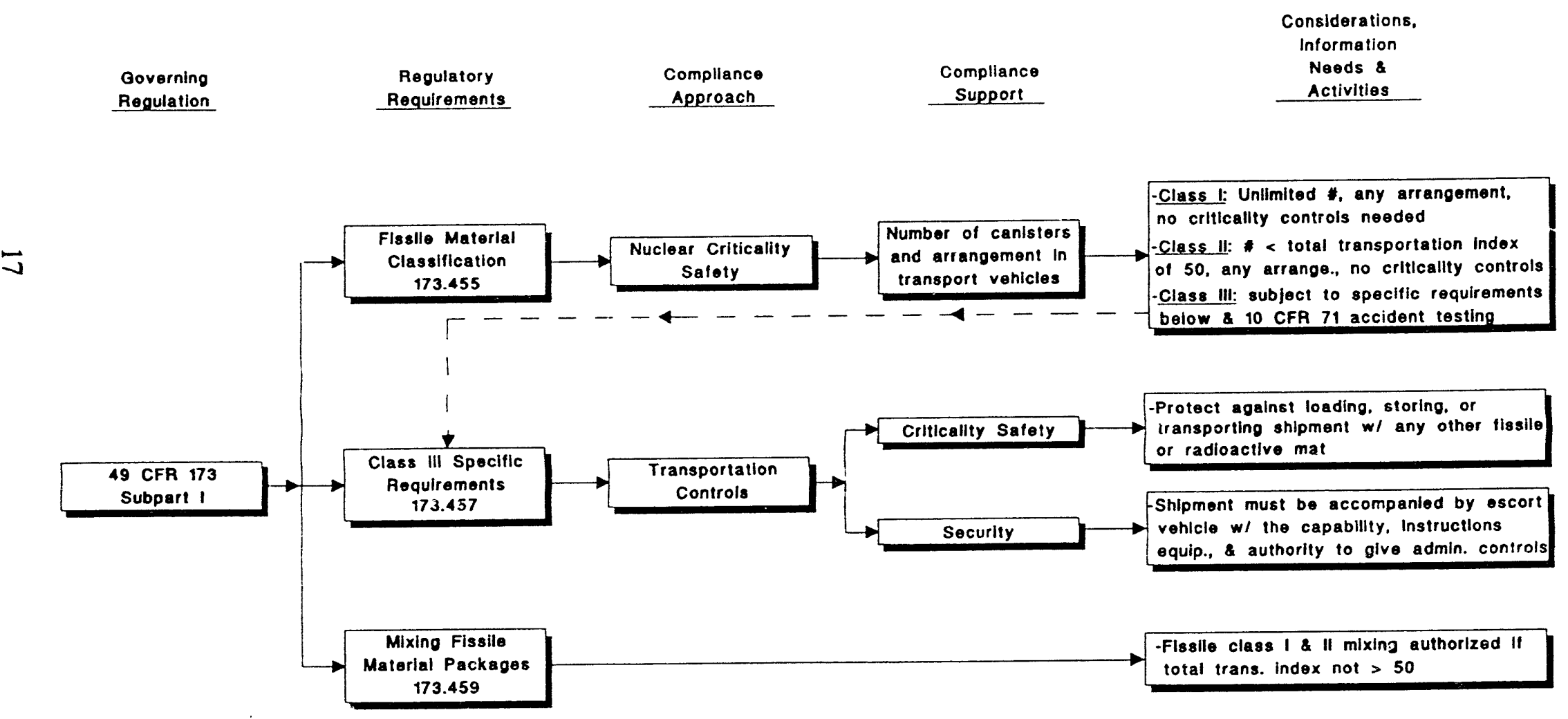




\section{CFR 173 Subpart I \\ Testing Requirements}

Governing

Regulation

Regulatory

Requirements
Compliance

Approach
Compliance

Support

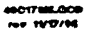

\begin{tabular}{c} 
Considerations, \\
information \\
Noods \& \\
Activitios \\
\hline
\end{tabular}

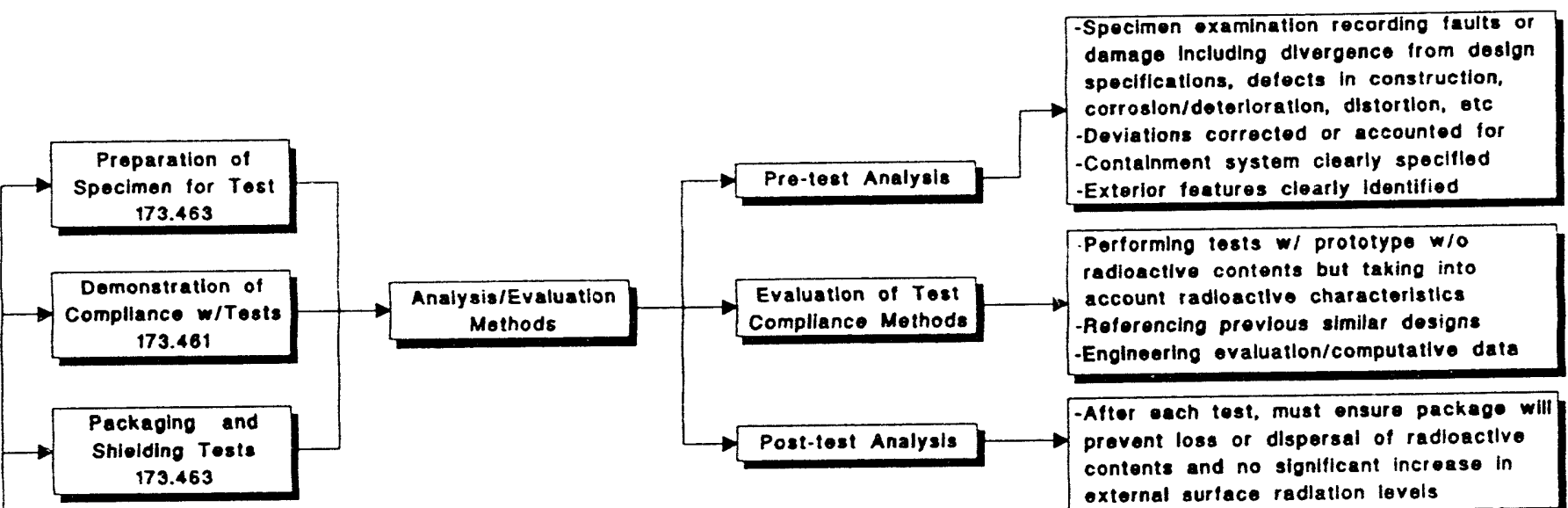

oxternal surface radlation levels

Type A: One prototype used for all tests Water Spray: precede esch tost or lest sequence; simulate raln $2 \mathrm{ln} / \mathrm{hr}$ for $1 \mathrm{hr} \mathrm{min}$ -Free Drop: fell onto target causing max damage to safoty features; $5000 \mathrm{~kg}$ or loss, distance dist. In tablo; Fissile Class II, drop test

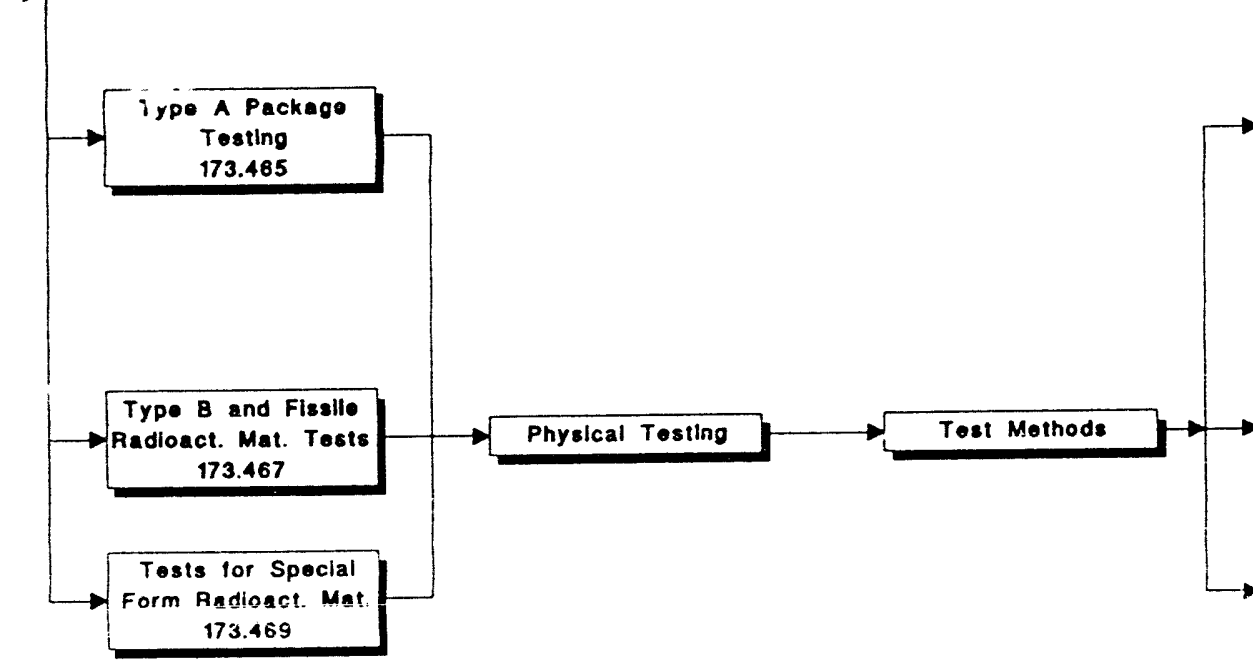
preceded by $3 \mathrm{~m}$ drop on each corner actual packege wolght or $1300 \mathrm{~kg}$ per sa $m$ multpliod by vertically prolected area - Penetration: package placed on rigld, flat aurface end $32 \mathrm{~cm}$ cylinder whemisphoro and welghing $6 \mathrm{~kg}$ vertically dropped $1 \mathrm{~m}$

Type B Add'l req: 10 CFR 71 Accident Cond (simultanous performance of tests) Speclal Fermi Different specimen each test Must not break/shatter during impact. percussion, or bending tesis

percussion, or bending tests

-Leaktlghtness \& indispersibllity checked -Test specifics: see 10 CFR 71 Subpart F llow diagram - special form tests 

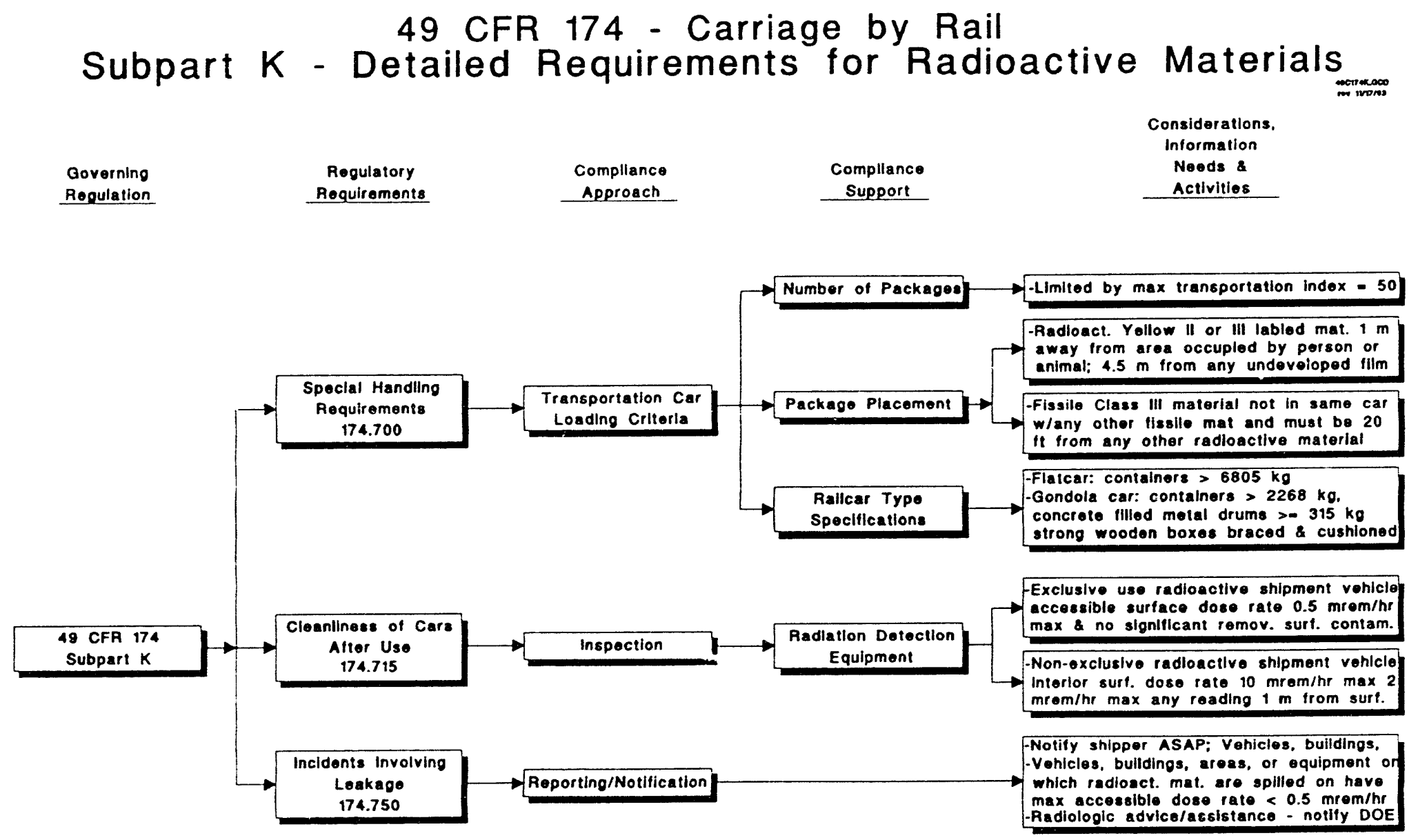


\section{CFR 263 - STANDARDS APPLICABLE TO TRANSPORTERS OF HAZARDOUS WASTE}

GOVERNING

REGULATION
Subparts

Subpart A General

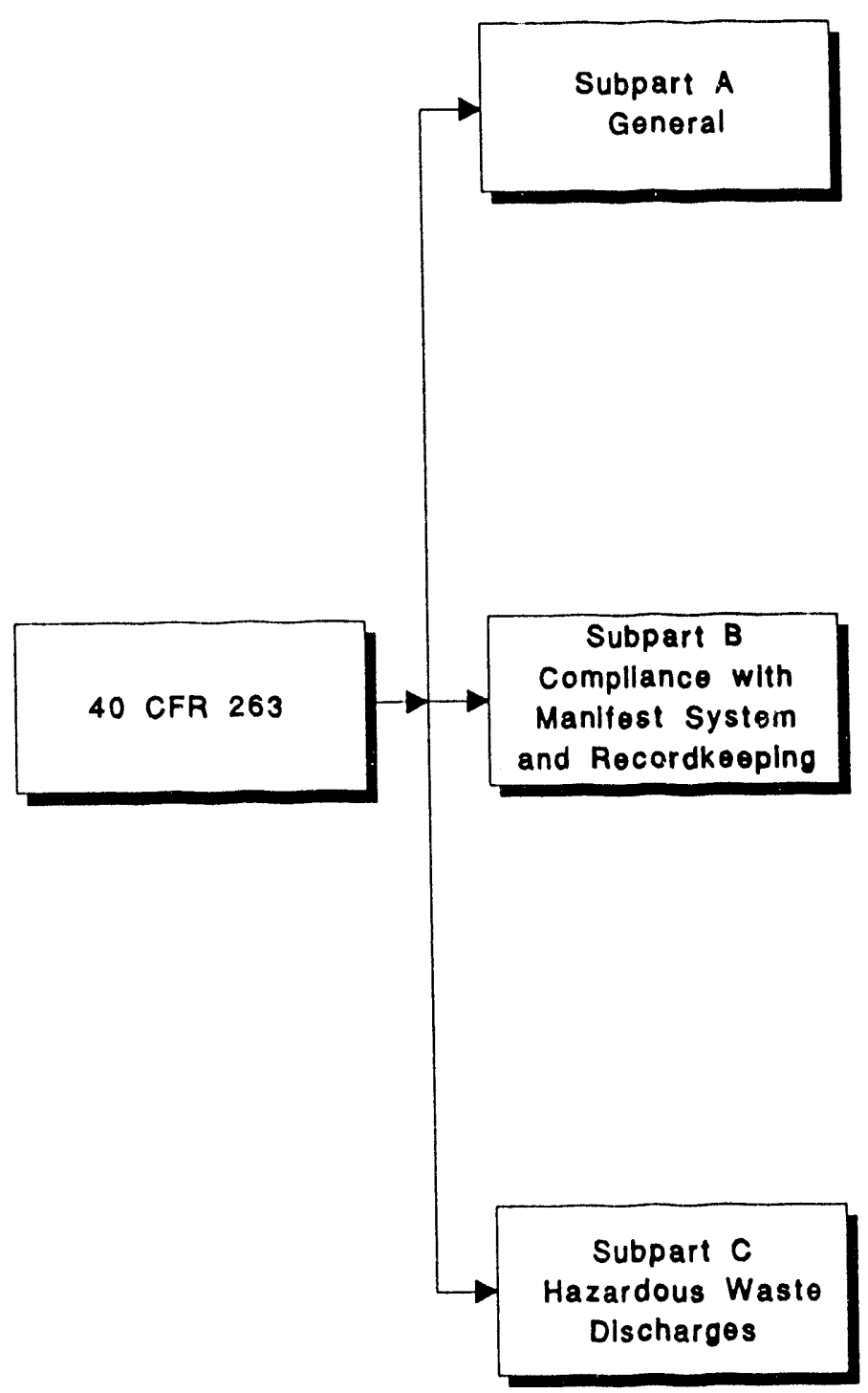




\section{DOE Order 1540.1A - Materials Transportation and Traffic Management

Governing Order

Procedures

Information

$N$

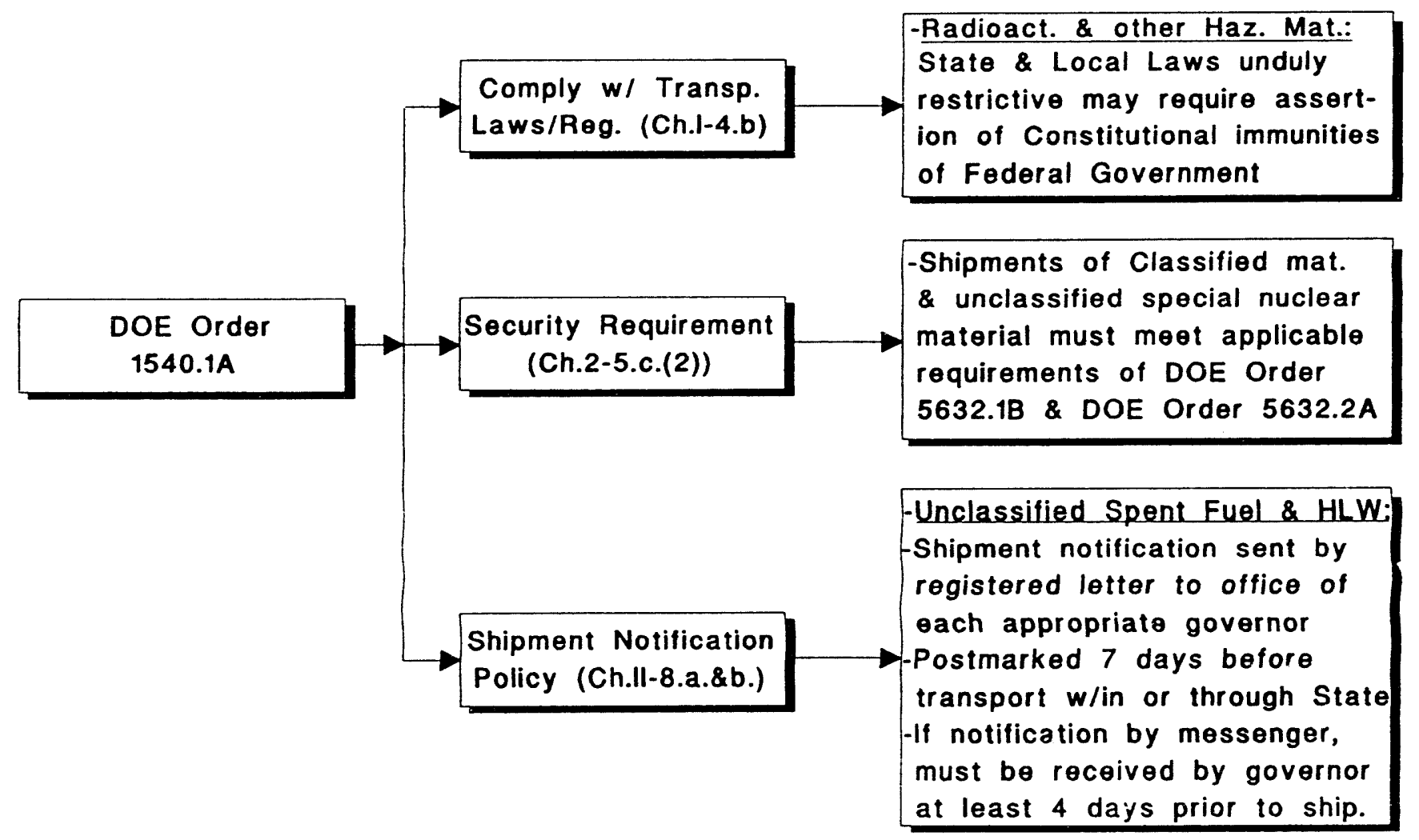


DOE Order 1540.2 - Haz. Mat. Packaging for Transport - Administrative Procedures

GOVERNING

REGULATION

Subparts

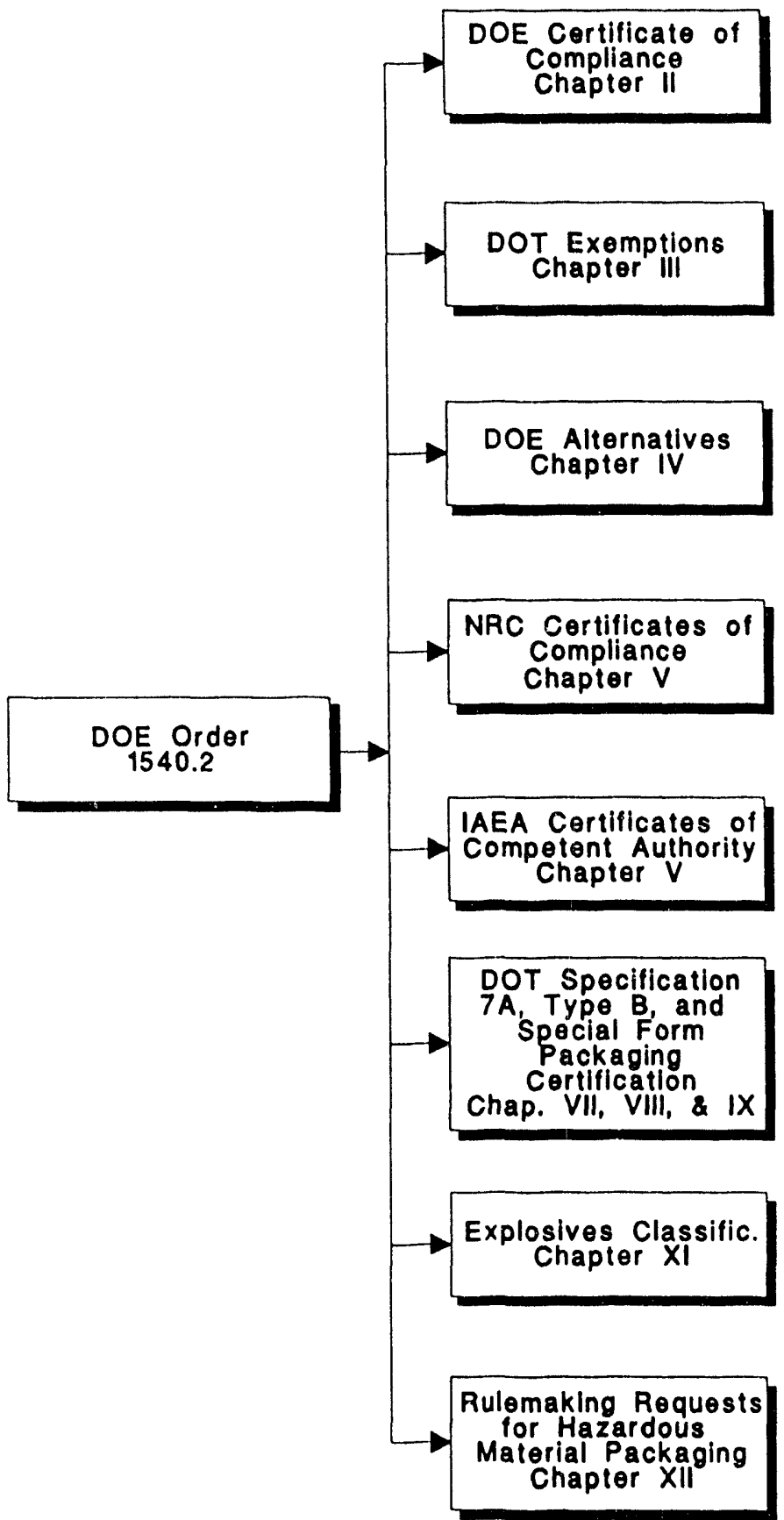




\section{DOE Order 5480.3 - Safety Requirements for Packaging \& Transportation of Hazardous Material, Substance, or Waste}

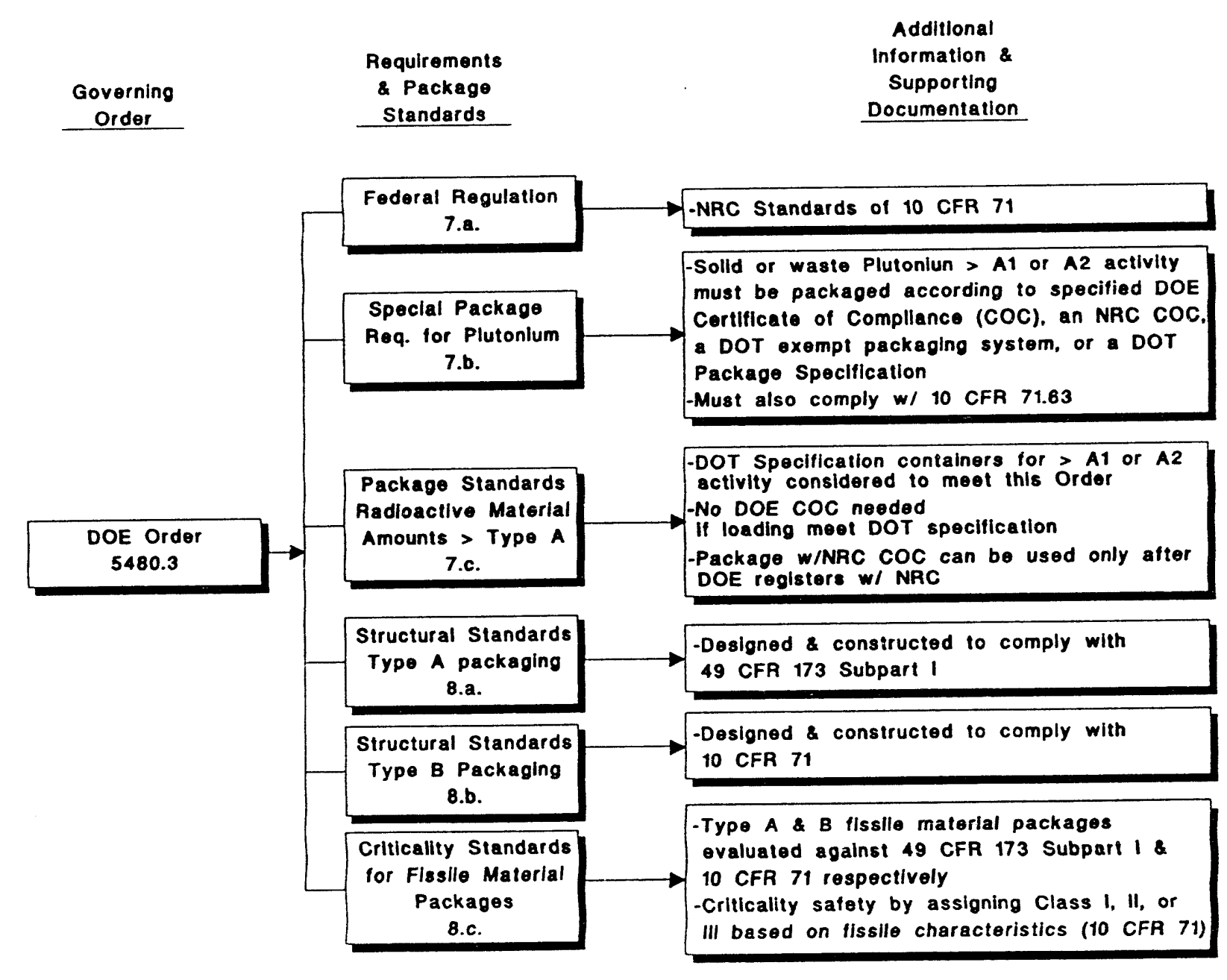


DOE-ID 5480.3 - Haz. Mat. Packaging and Trans. Safety Req. Attachment 1 - Chapter I: Off-site Shipments

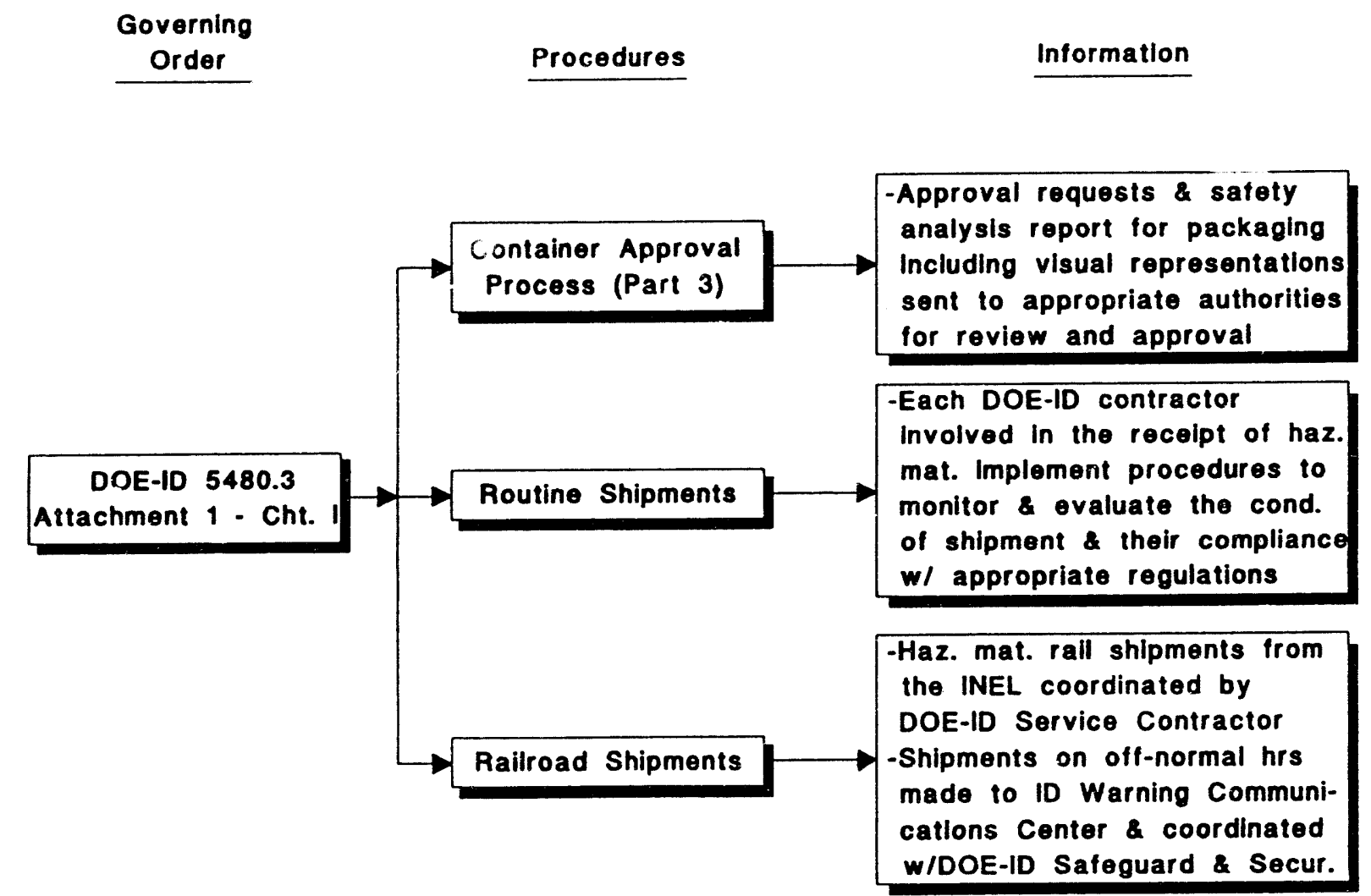




\section{DOE-ID 5480.3 - Haz. Mat. Packaging and Trans. Safety Req.}

Attachment 1 - Chapter II: On-site Transfers ont ontoco

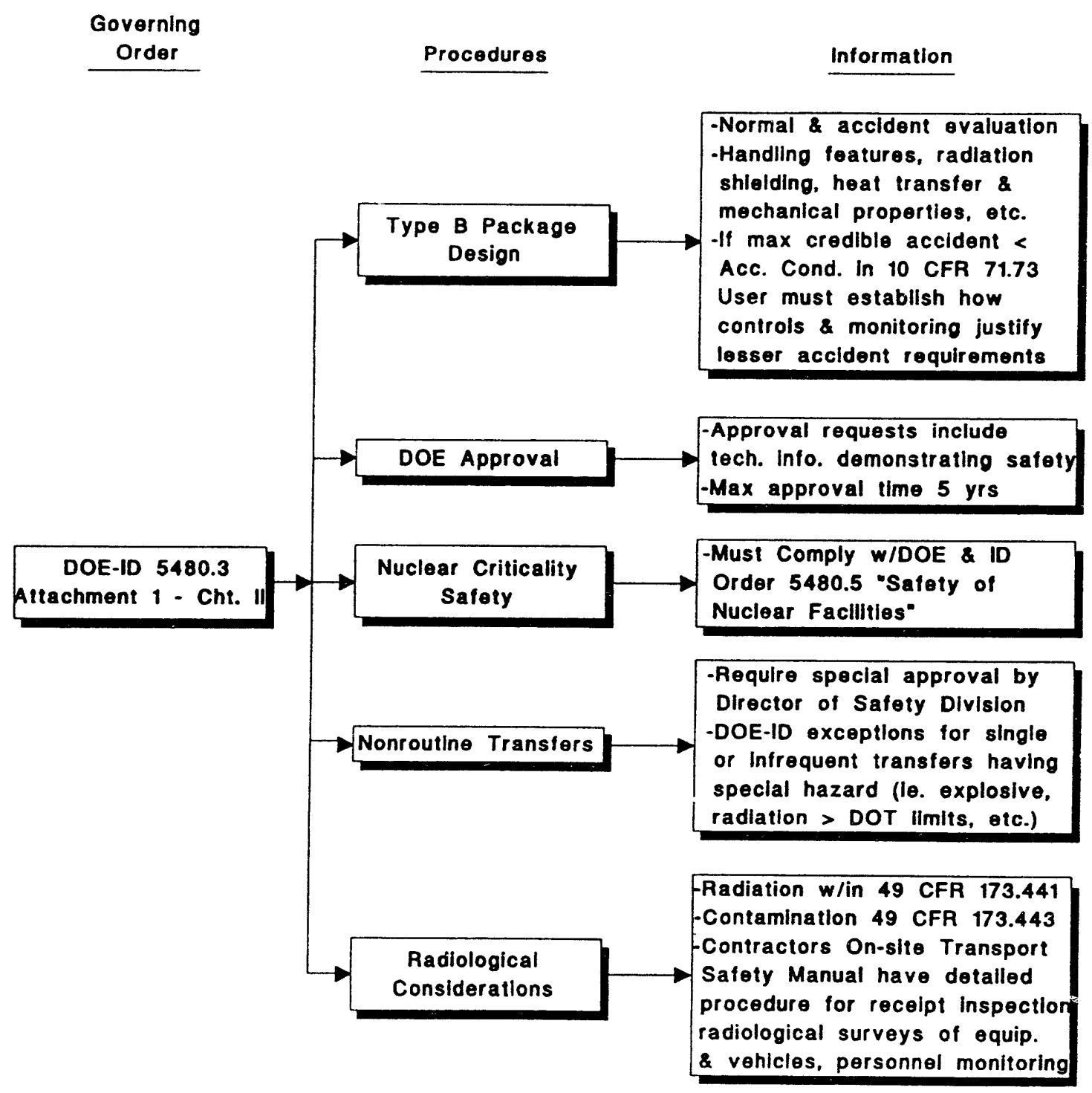




\subsection{MGDS Requirements}

Under Title 40 of the Code of Federal Regulations, section 191 (40 CFR 191), all Mined Geologic Disposal Sites (MGDS) "shall be designed to provide a reasonable expectation, based on performance assessments (PA), that the cumulative releases of radionuclides to the accessible environment for 10,000 years after disposal" will not exceed the release limits outlined in 40 CFR 191.13. A Performance Assessment, as defined, "(1) identifies the processes and events that might affect the disposal system; (2) examines the effects of these processes and events on the performance of the disposal system; and (3) estimates the cumulative releases of radionuclides, considering the associated uncertainties, caused by all significant processes and events." With this in mind, section 3 summarizes the regulatory requirements for conducting a PA and outlines the nuclear waste packaging requirements that will ensure compliance with both the transportation requirements (discussed in Section 2.0) and MGDS restrictions.

A summary of general aspects that must be accounted for in the development of PA models (or other analysis methods) has been included as the first flow diagram in section 3.2; this is an overall summary of the key performance factors that are addressed throughout the regulations in this section. Other flow diagrams encompass the technical aspects involved in siting, licensing, release limits, waste interactions with the container, container design features, physical characteristics of the surrounding environment, facility design features, barrier systems, safety features, implementation guidelines, migration restrictions, criticality considerations, model verification methods, and so forth.

Since many of the factors influencing the long-term performance of a MGDS are site-specific, a complete assessment of the long-term performance of waste in the MGDS cannot be conducted until after an actual site has been selected and fully characterized for repository development. This section is intended to aid in the modeling development for a hypothetical geologic repository, accounting for as many factors as possible, until such time when an actual MGDS is identified.

\subsection{Listing of Regulations and Orders Developed}

Summary of General Aspects Implied by Performance Assessment Modeling \& Analysis Compliance Approach

10 CFR 60 Disposal of High Level Radioactive Waste in Geologic Repositories

Subpart B - Licenses (Summary)

Sections: - Licenses Compliance Structure

- Construction Authorization \& Permanent Closure Compliance Structure 


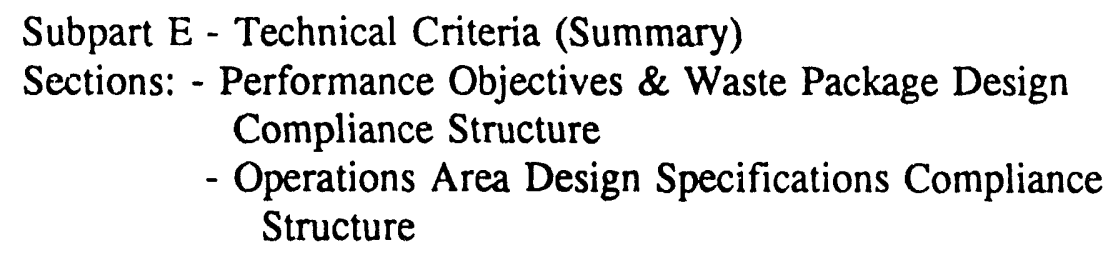

10 CFR 960 General Guidelines for the Recommendation of

Sites for Nuclear Waste Repositories

Subpart B - Implementation Guidelines

Subpart C - Postclosure Guidelines (flow diagram)

- Postclosure Technical Guidelines Table

Subpart D - Preclosure Guidelines (flow diagram)

- Preclosure Technical Guidelines Table

40 CFR 191 Subpart B - Environmental Standards for the Uranium Fuel Cycle Compliance Structure

40 CFR 264 Subpart X - Permit for Misc. Unit Compliance Structure

40 CFR 268.6 Land Disposal Restriction Compliance Structure

10 CFR 20 Standards for Protection Against Radiation

DOE Order 5820.2A - Radioactive Waste Management (Update in process): Chapter I - High Level Waste; Section 3d - Disposal Requirements

\subsection{Flow Diagrams for Regulations and Orders}

Flow diagrams are in the order listed in section 3.1. 


\section{Summary of General Aspects Implied by \\ Performance Assessment Modeling \& Analysis Compliance Approach

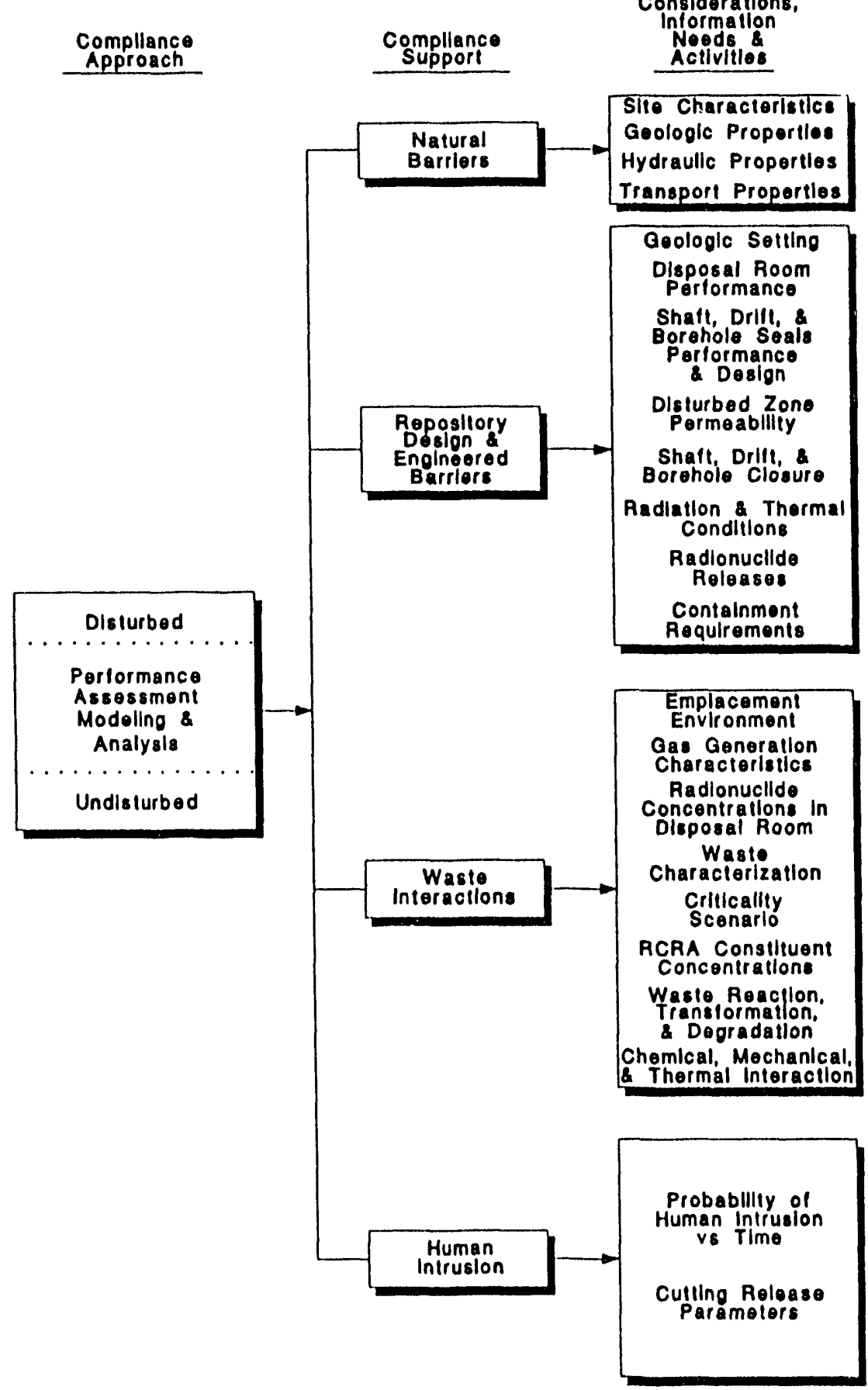

NOTE: Porformanco Assossment Modellng Analysis, whon used as a Compllanco Approach in flow diagrams indireclly Implles all of the above aspocts. Supporting requirements in these areas alo covered by the flow diagrame for the spoclic regulations in which these aspocts are containod. 


\section{CFR 60 DISPOSAL OF HIGH LEVEL RADIOACTIVE WASTES IN GEOLOGIC REPOSITORIES}

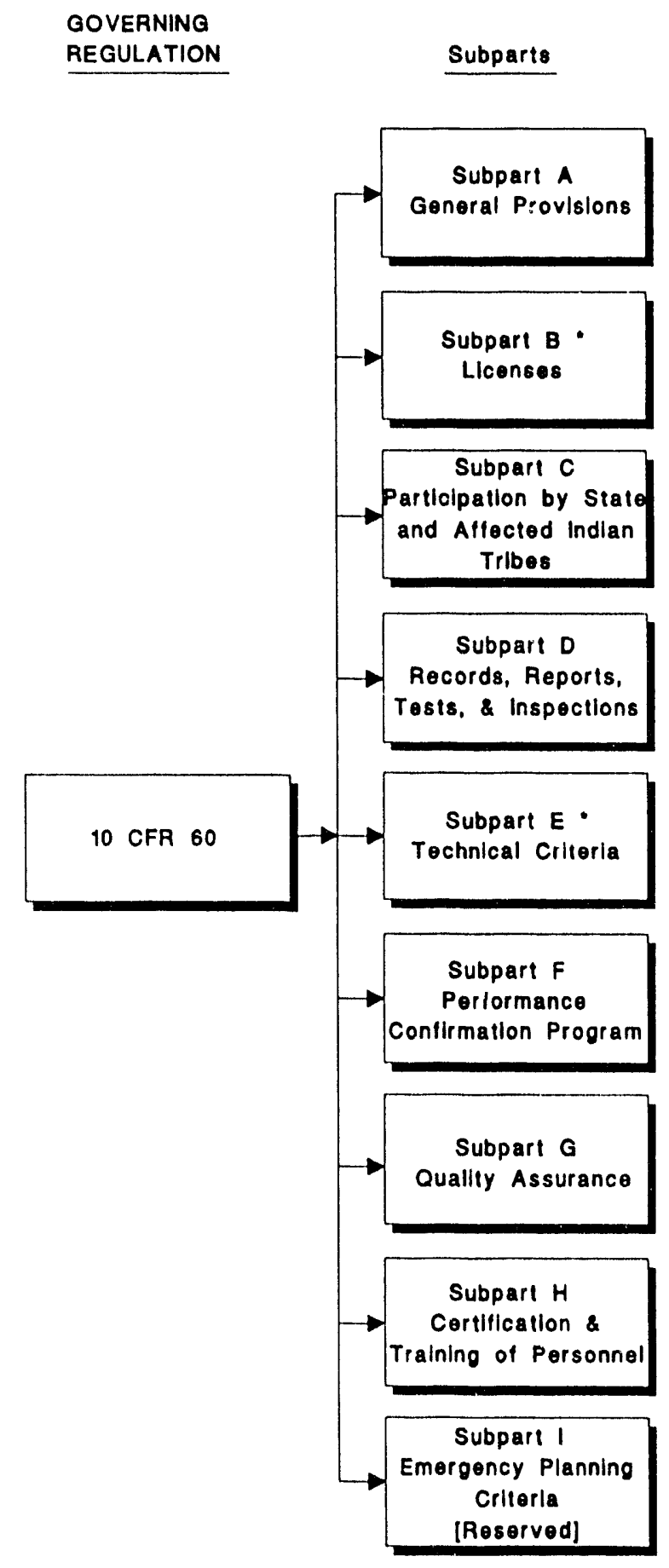

- Indicales that a flow degram for this aubpart has been devolopod. 


\section{CFR 60 Subpart B Licenses Compliance Structure (Summary)}

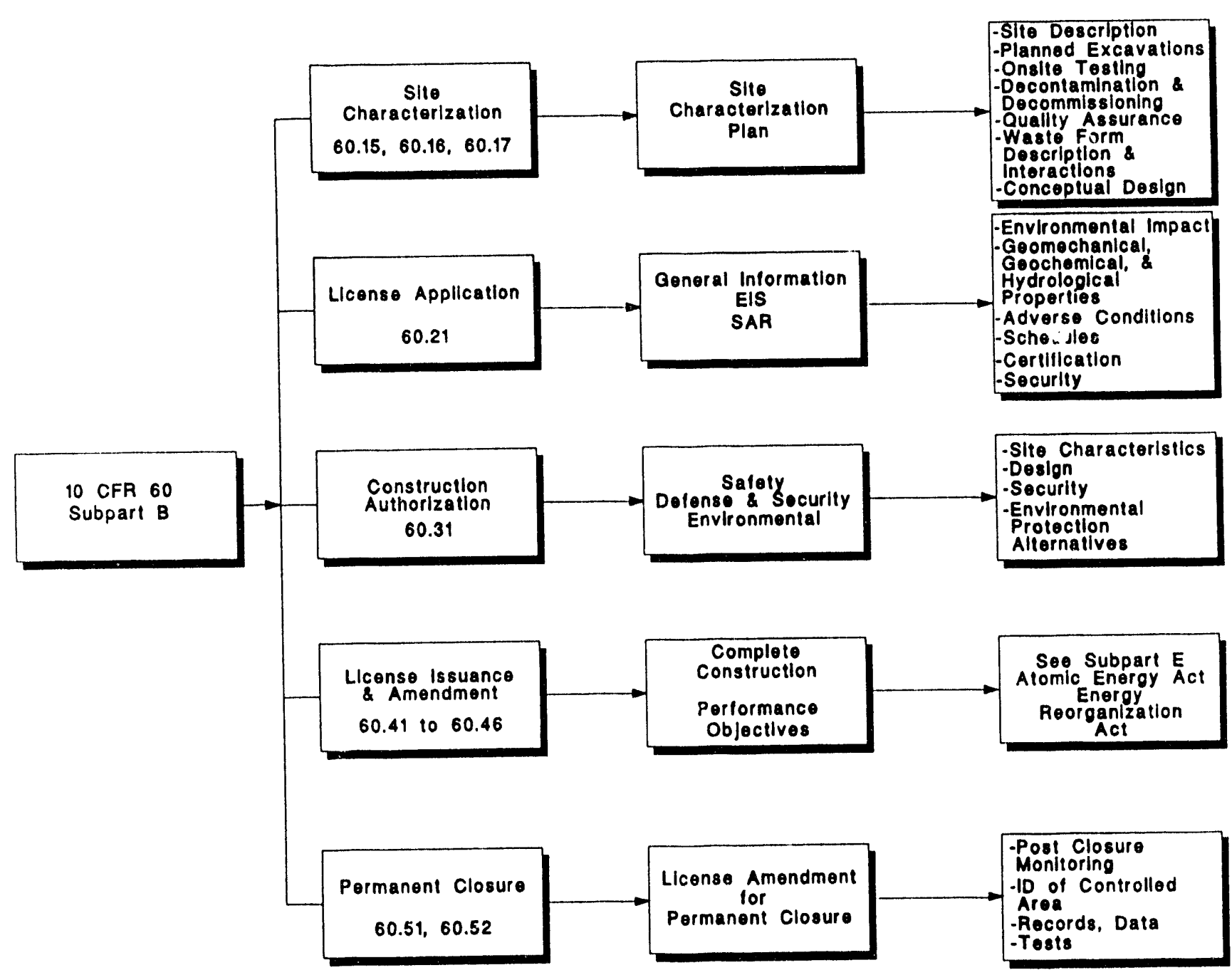


10 CFR 60 Subpart B

Licenses Compliance Structure

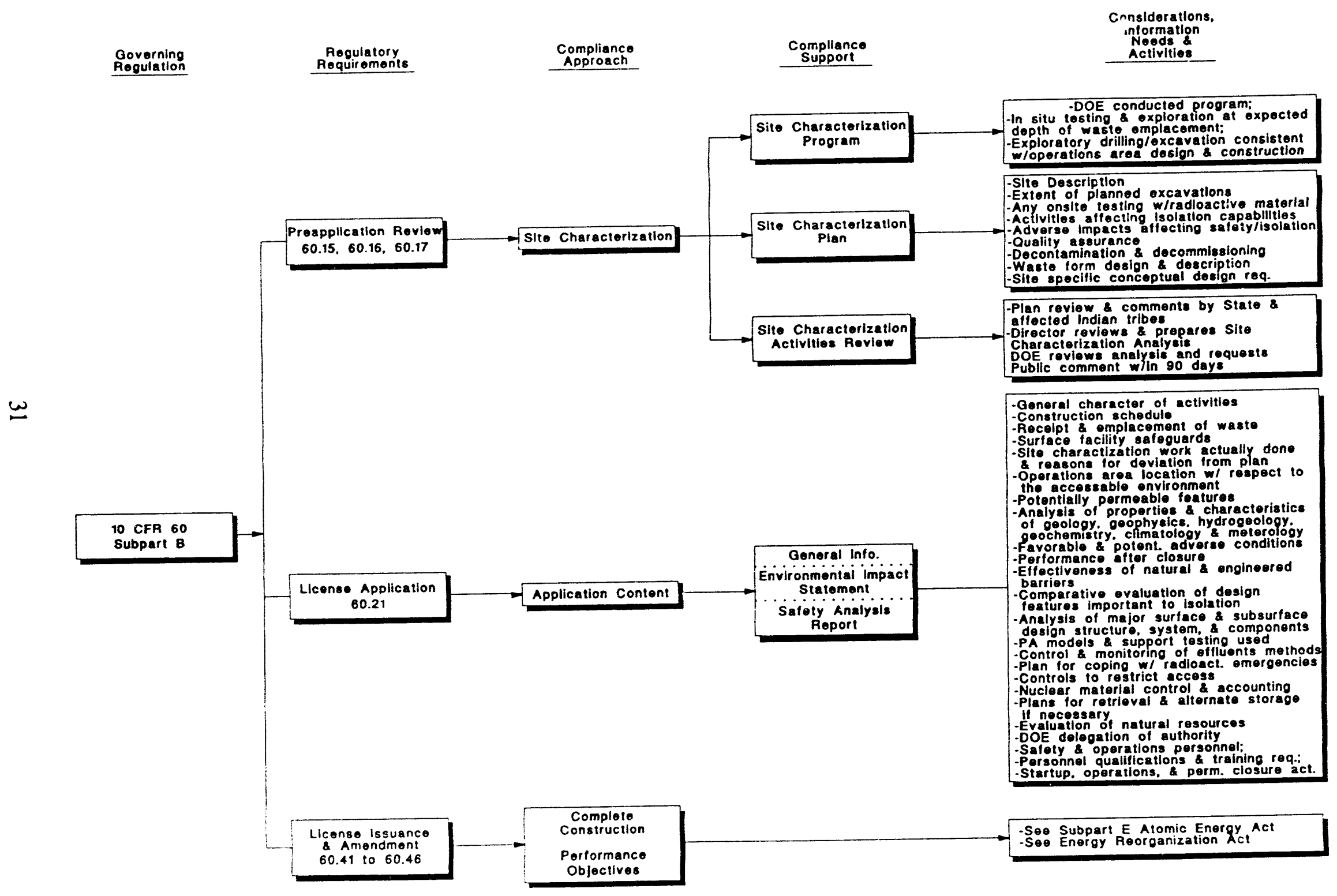


10 CFR 60 Subpart B

scosecoso

Construction Authorization \& Permanent Closure Compliance Structure

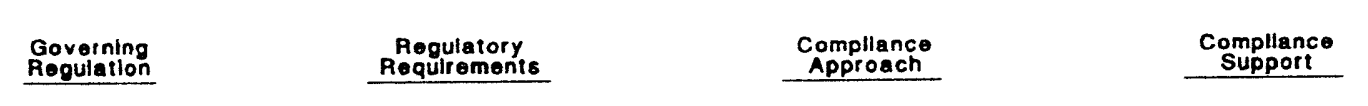

Considerations,

Information
Needs \&

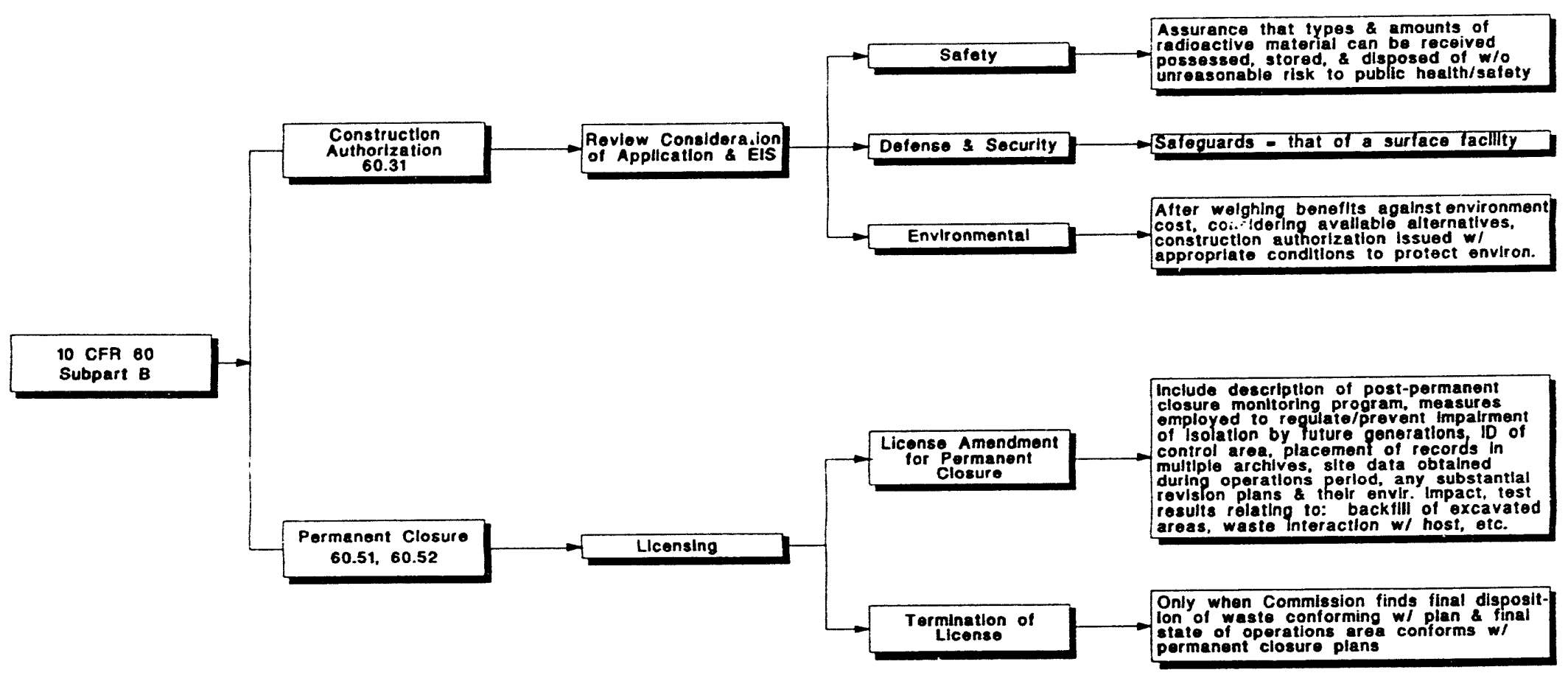




\section{CFR 60 Subpart E Technical Criteria Compliance Structure (Summary)}

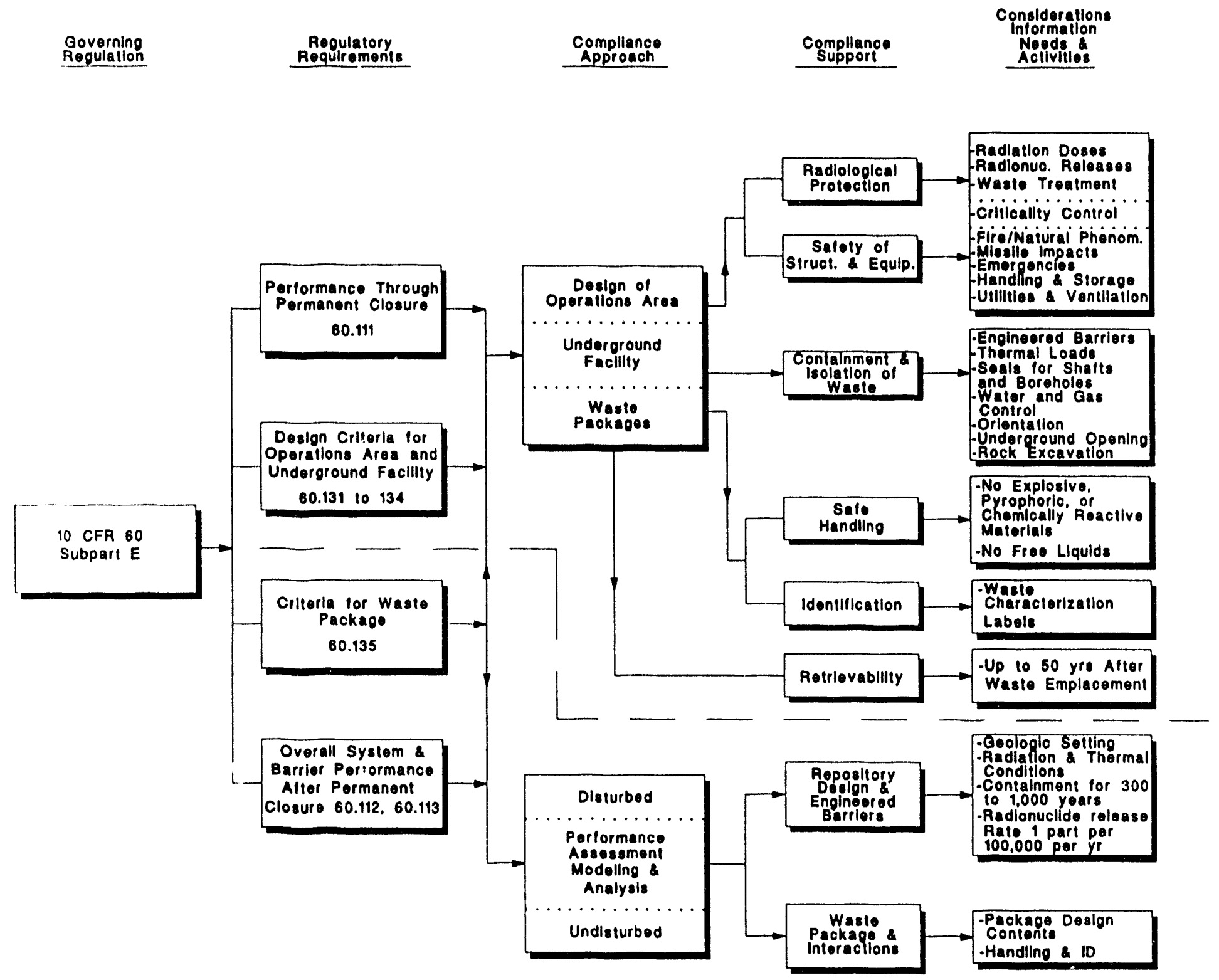


10 CFR 60 Subpart E

Technical Criteria - Performance Objectives \& Waste Package Design Compliance Structure

Complliance

Considerations \begin{tabular}{l} 
Noeds \\
Activitios \\
\hline
\end{tabular}

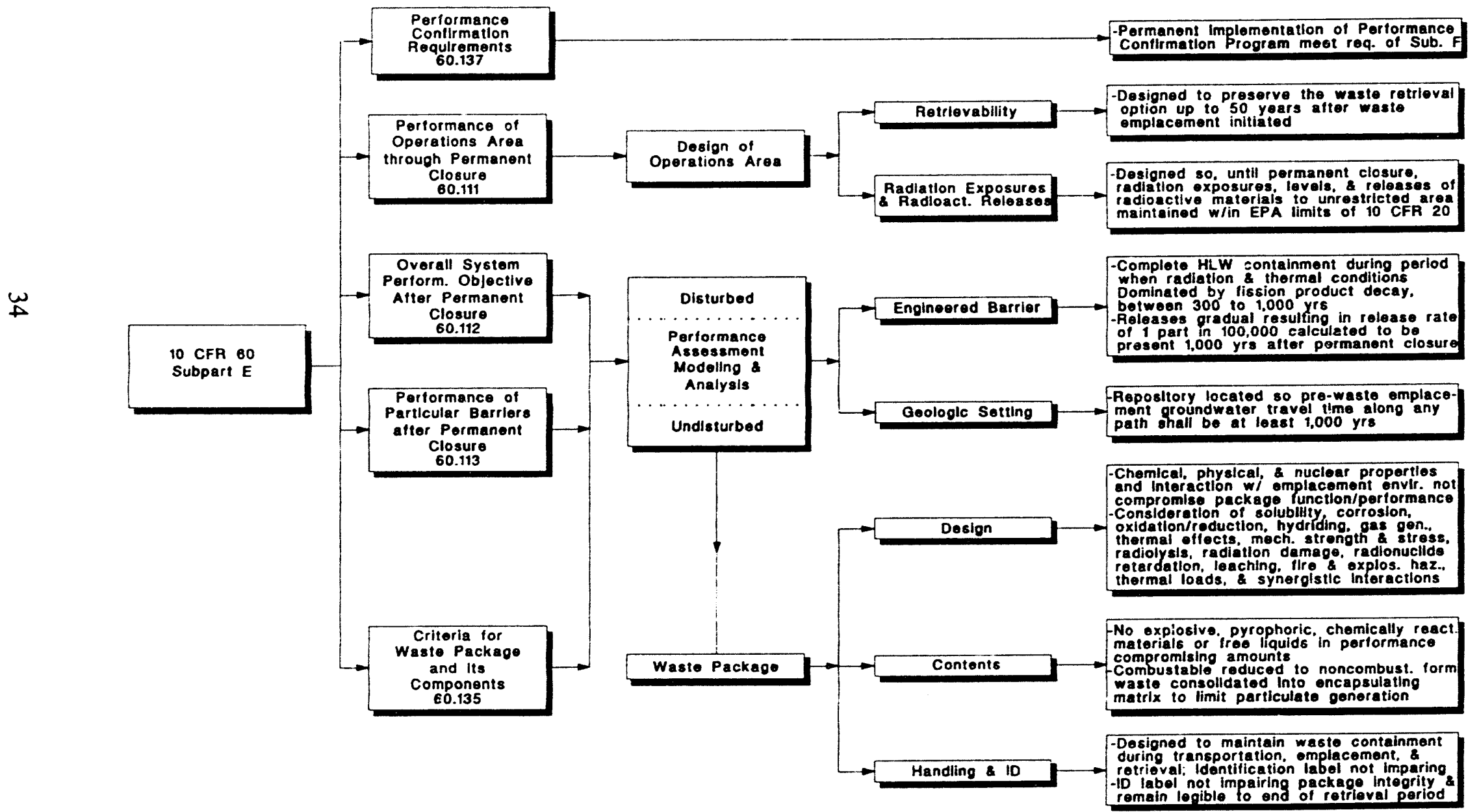


10 CFR 60 Subpart E

Technical Criteria - Operations Area Design Specifications Compliance Structure

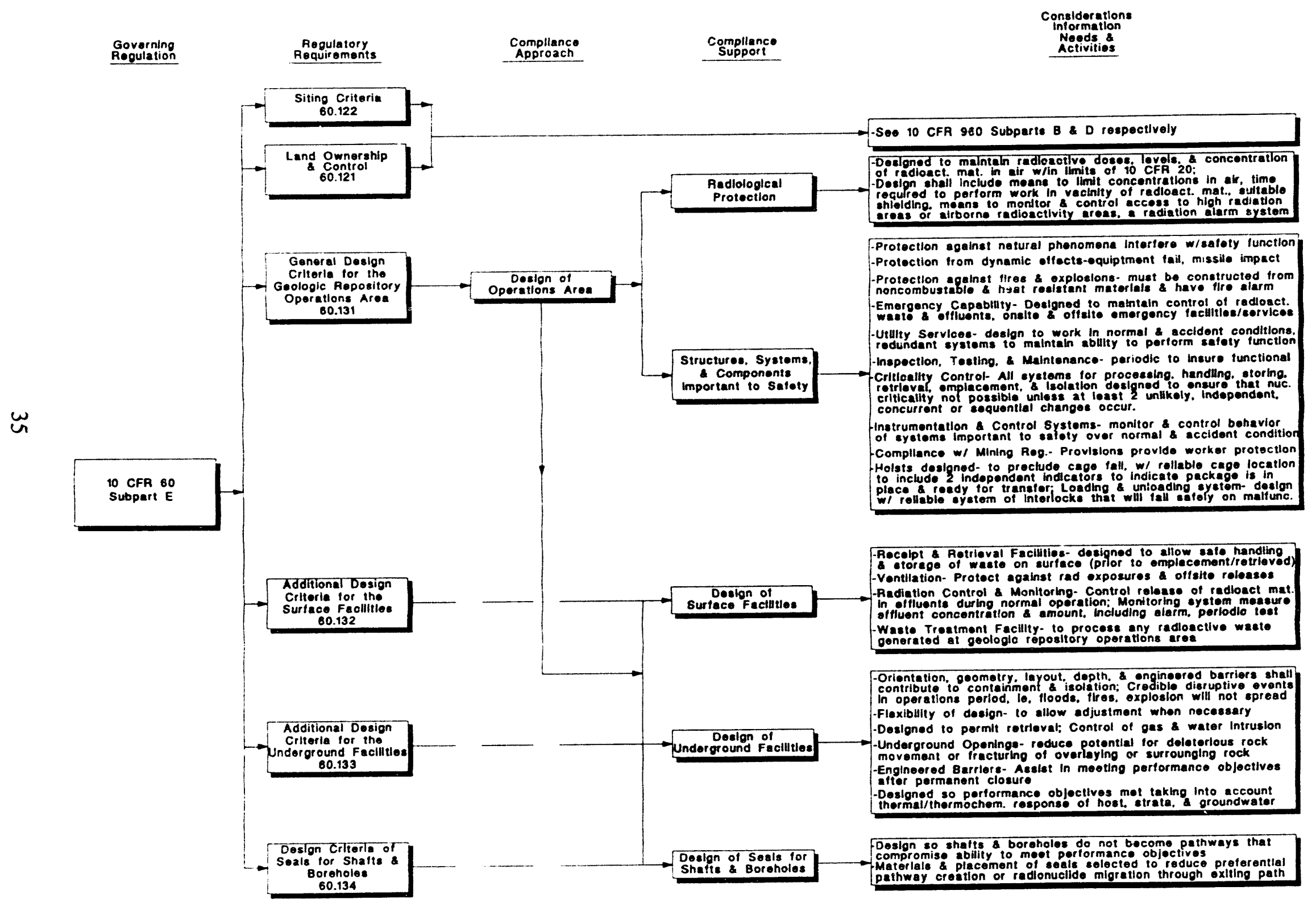




\section{CFR 960 General Guidelines for the Recommendation of Sites for Nuclear Waste Repositories = \\ Governing \\ Aogulation \\ Subpints}

Subpurt A

General frovisione

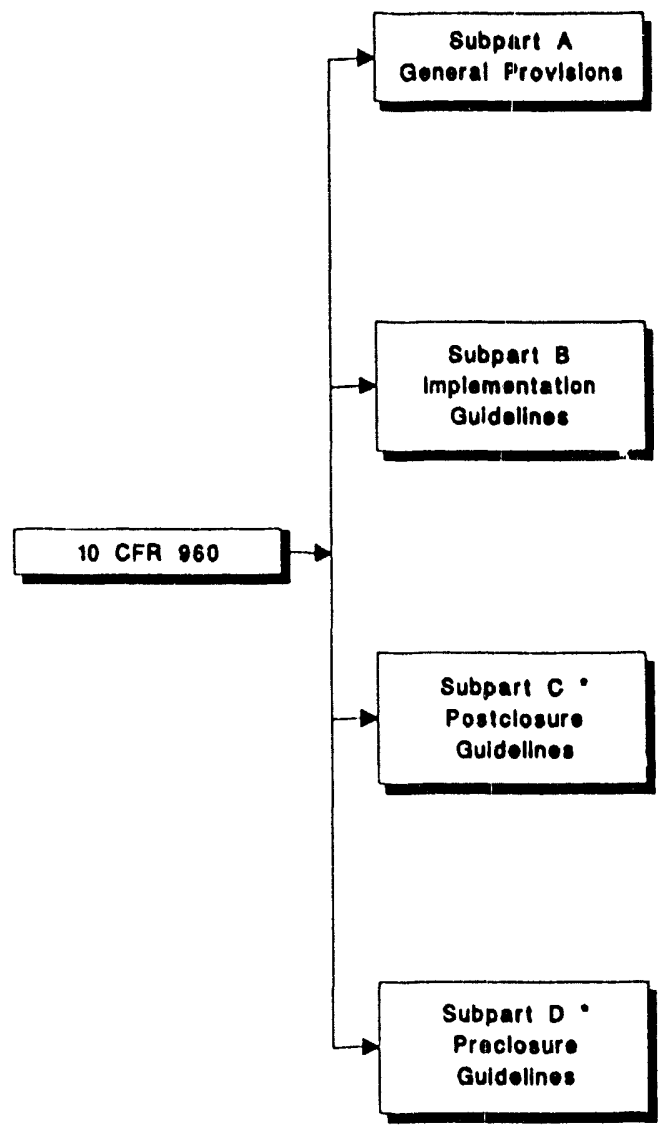

- Indlcates that a flow dlagram for thls subpart has been doveloped 


\section{CFR 960 Subpart B Implementation Guidelines}

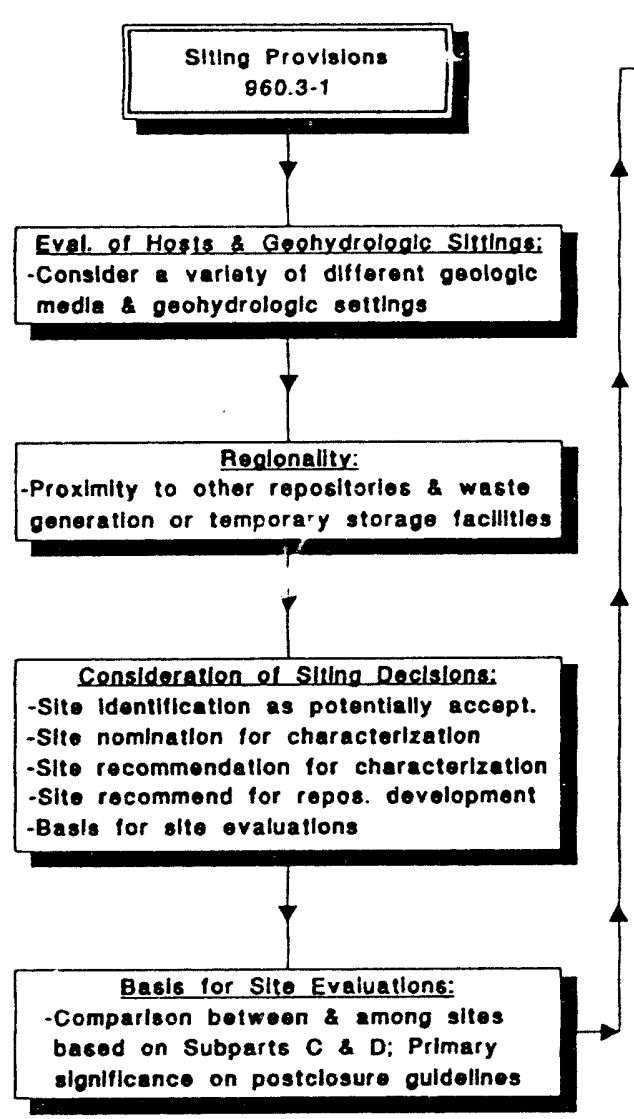

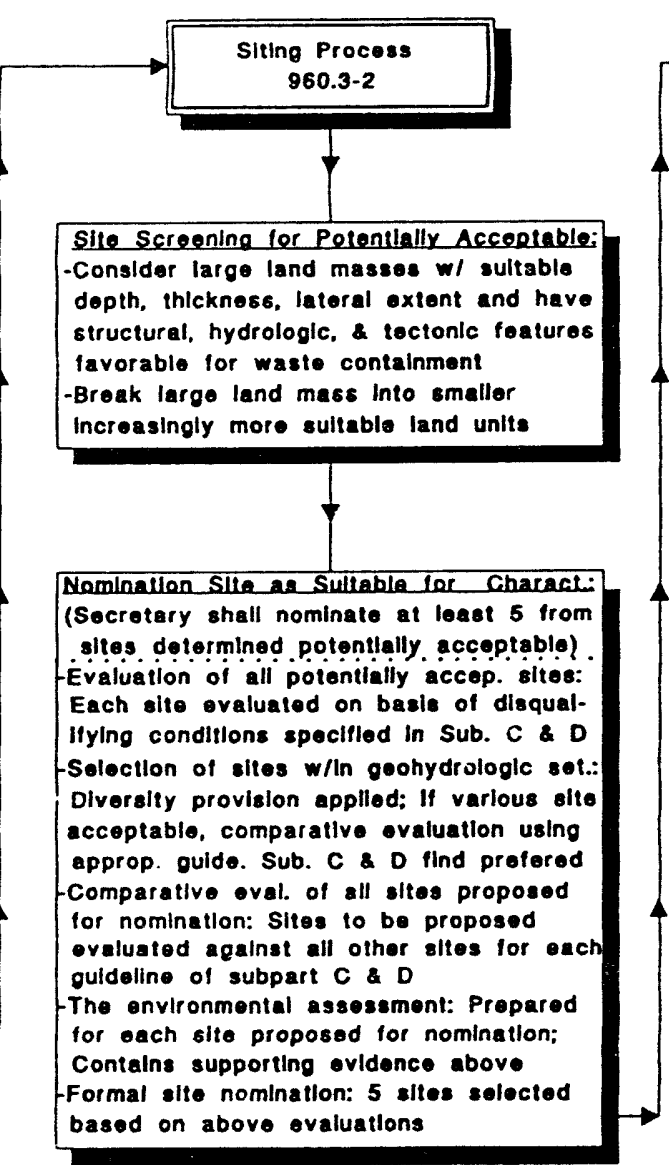

Becommendation slles for Characterization: After 5 nominated, Secretery recommend In writing min of 3 to President; Declsion besed on avallable geophysical, geologic, geochemical, \&ydrologic data, other Into essociated ovaluations a findings

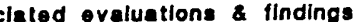

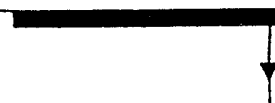

Recemmendallon of Sile for Repes Devel: After characterlzation, candidate sltes are compared w/ each other on the besis of Sub. C D according to siting provision specifying the basis for ovaluations.

loading to recommendation by

Secretary to the Prosident

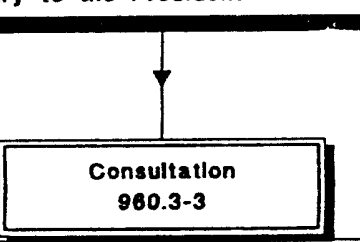

DOE provide alfected States/indian tribes ilmoly \& complete Info.: written response o requests returned to DOE w/in 30 days

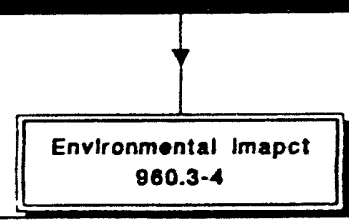

impacts shall be considered throughout the site characterization, solection, ropos. develop. proc.; DOE mitlgate neg. Impact 


\section{CFR 960 Subpart C Postclosure Guidelines}

Consideratlons,

Information

Neods \&

Governing

Regulatory

Compliance

Compllance

Regulation

Requirements

Approach

Support

Activilles

Goologlc selting mus

allow for physical

separation of wasto

from the accessible

onvironment after

closure according to

40 CFR 191 Sub. B

$\omega$

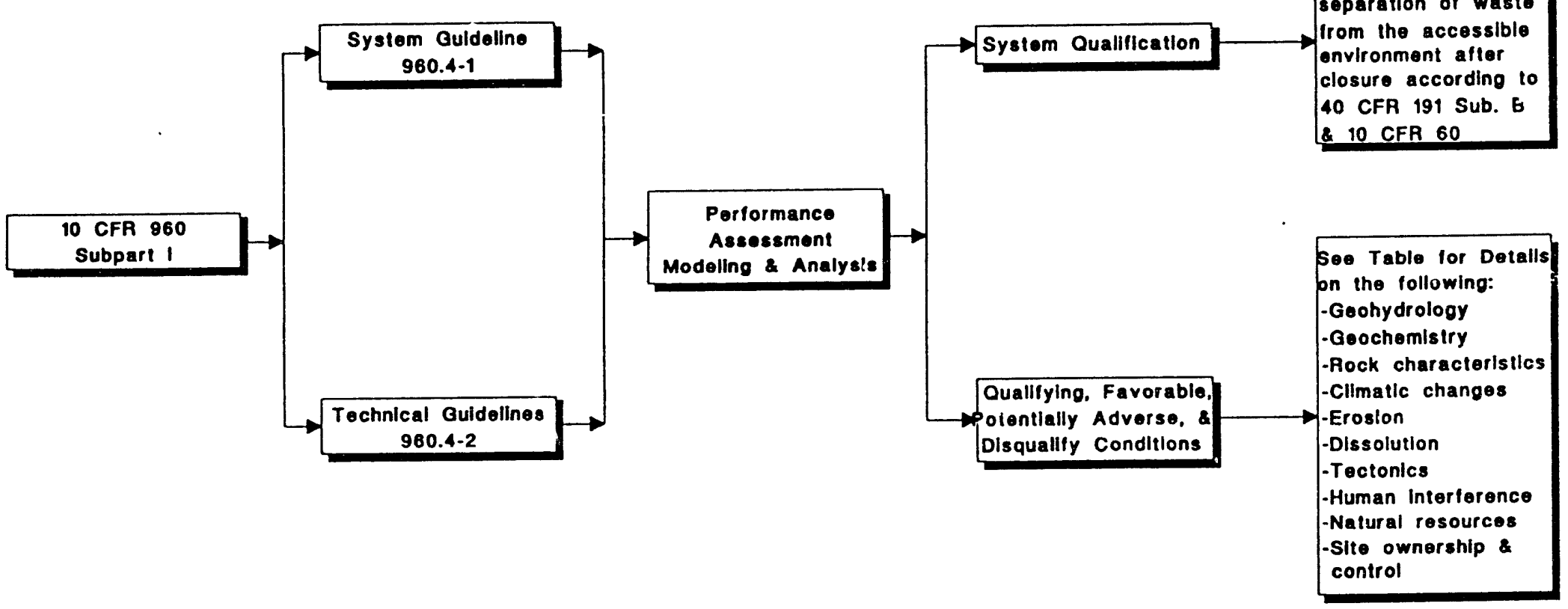


10 CFR 960 Subpart C

Postclosure Technical Guidelines Table

\begin{tabular}{|c|c|c|c|c|}
\hline Tochmlenl Guldaline & Qualitying Condilion & Fevorublio Condition & Potentlelly Acterceo Comaltione & Dlequelli ying Condittone \\
\hline $\begin{array}{l}\text { Goohydrotoey } \\
900 \cdot 4-2-1\end{array}$ & 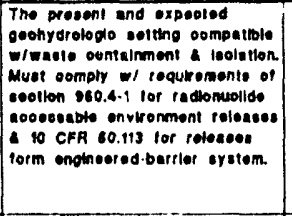 & 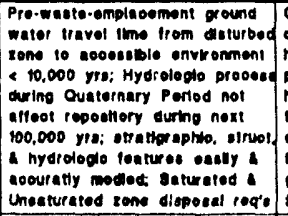 & 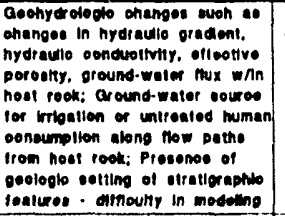 & 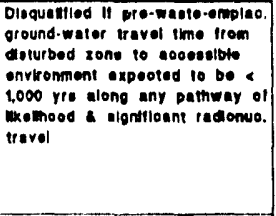 \\
\hline $\begin{array}{l}\text { Goochomlatry } \\
\text { Oo0.4.2.2 }\end{array}$ & 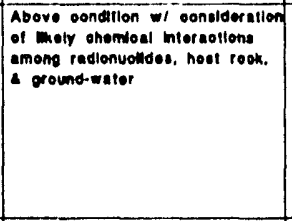 & 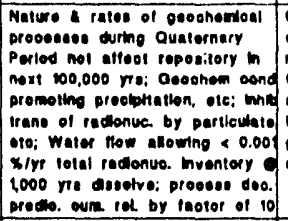 & 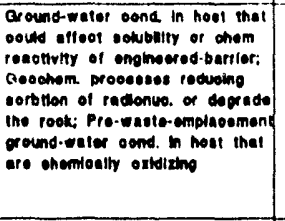 & none \\
\hline 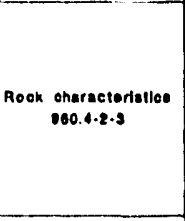 & 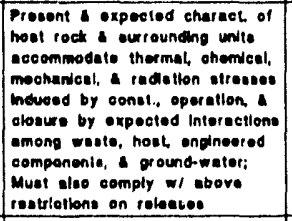 & 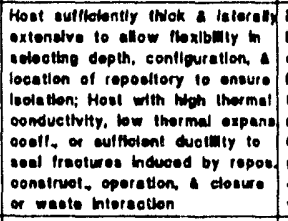 & 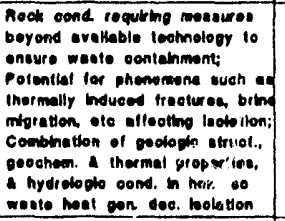 & none \\
\hline $\begin{array}{c}\text { Cimatle onenges } \\
0.0 .4-2 \cdot 4\end{array}$ & 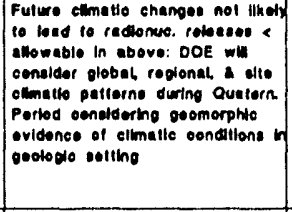 & 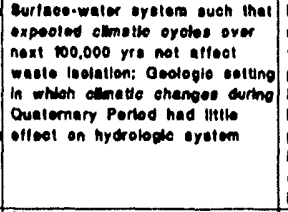 & 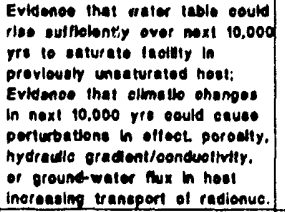 & none \\
\hline $\begin{array}{c}\text { Eropion } \\
\text { PBo.4.2.5 }\end{array}$ & 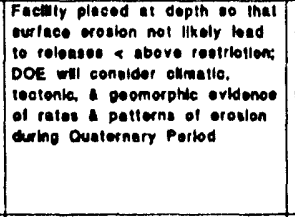 & 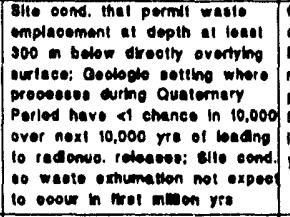 & 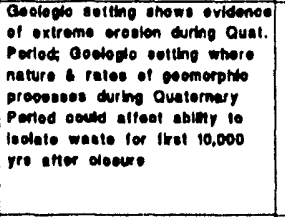 & 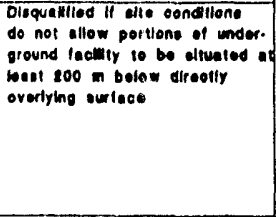 \\
\hline $\begin{array}{l}\text { Dicootulion } \\
060.4-2-6\end{array}$ & 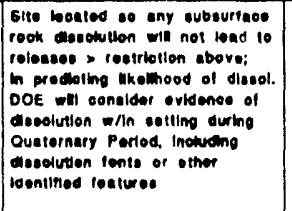 & 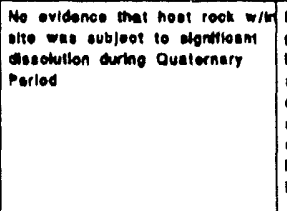 & 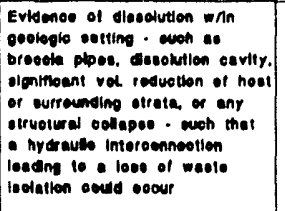 & 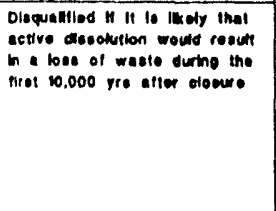 \\
\hline $\begin{array}{l}\text { Tectonles } \\
\text { Deso.4.2.7 }\end{array}$ & 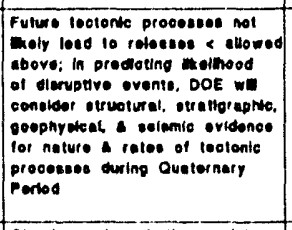 & 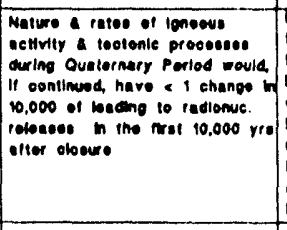 & 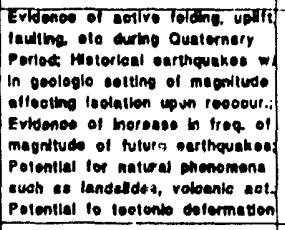 & 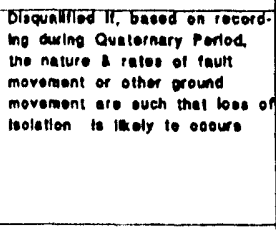 \\
\hline $\begin{array}{c}\text { Muman Imtertorenoe } \\
\text { Pe0.4-2-8 }\end{array}$ & 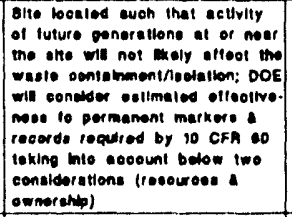 & nono & none & none \\
\hline 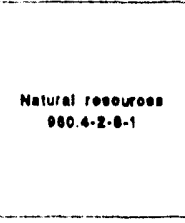 & 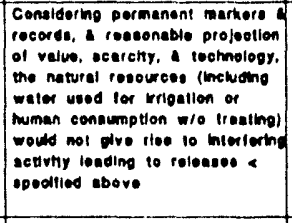 & 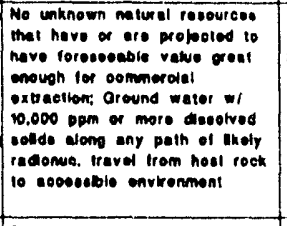 & 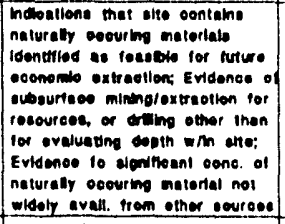 & 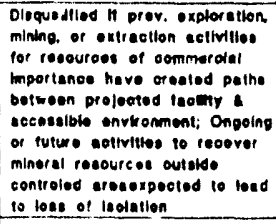 \\
\hline $\begin{array}{l}\text { Olle Ownocetip } \\
\text { A Conirol } \\
\text { Oto } 4 \cdot 2 \cdot 0-2\end{array}$ & 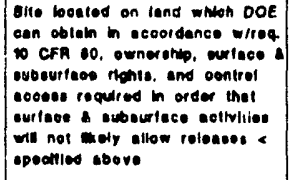 & 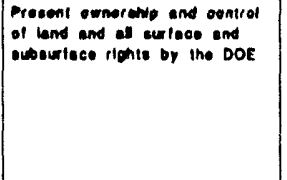 & 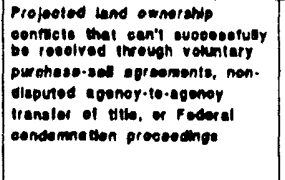 & none \\
\hline
\end{tabular}

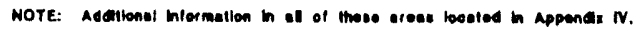




\section{CFR 960 Subpart D Preclosure Guidelines}

Considerations,

Information

Neods a

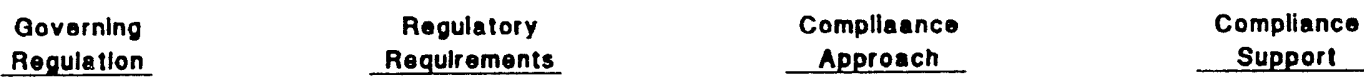

Activitios
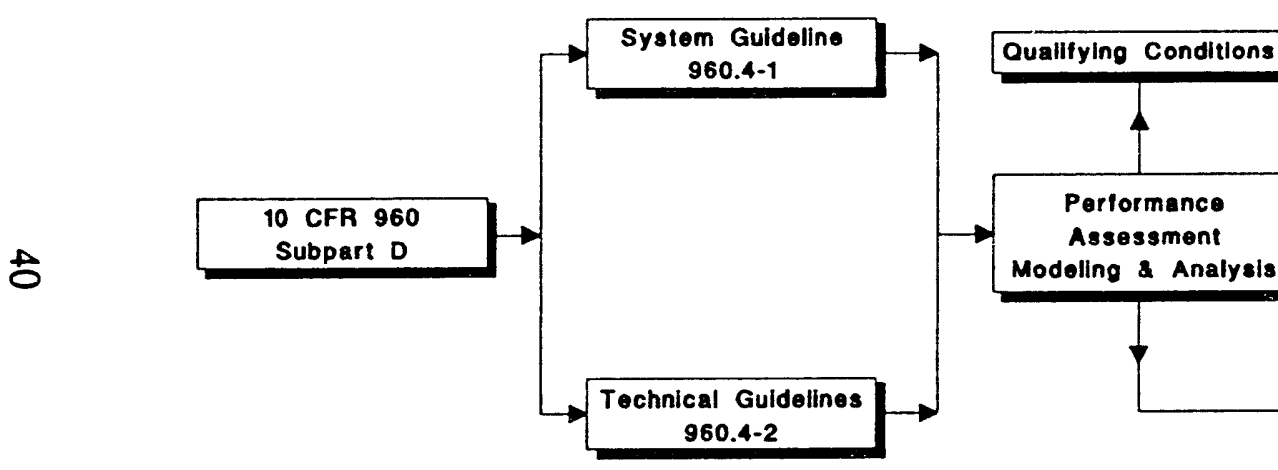

Environmental, Socioeconomics.

protect from hazards
from siting-decom.

Modoling a Analysis

a Transportation

\section{Easo and Cost of \\ From siting-closure} Siting. Construction Operation, \& Closur.

must be foasible

tochnologically

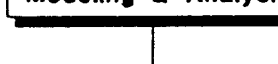

ochnical criteria

set forth quallfying.

Technical Guldelines
Purpose and Scope $\longrightarrow \begin{aligned} & \text { favorable, potentlally } \\ & \text { adverse, a disquallf. } \\ & \text { cond. on performance }\end{aligned}$

Technical Guldelines
Purpose and Scope $\longrightarrow \begin{aligned} & \text { favorable, potentlally } \\ & \text { adverse, a disquallf. } \\ & \text { cond. on performance }\end{aligned}$

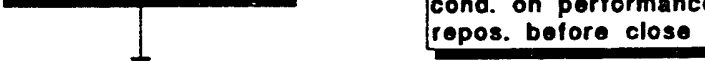

\begin{tabular}{|c|c|}
\hline $\begin{array}{l}\text { Toples divided Into } \\
\text { following } 3 \text { areas in } \\
\text { docroesing order of } \\
\text { Importance: } \ldots \ldots \ldots \\
\text {-Procilosurg }\end{array}$ & $\begin{array}{l}\text {-Pop. donsity \& dist. } \\
\text { - Sile ownership \& } \\
\text { control } \\
\text { - Moterology } \\
\text { - Offsite installations } \\
\text { and operations }\end{array}$ \\
\hline $\begin{array}{l}\text { Radlological Safety } \\
\text {-Environmontai, } \\
\text { Soclooconomics. } \\
\text { Transportatlon . . }\end{array}$ & $\begin{array}{l}\text {-Environmental quality } \\
\text {-Soclooconom. impaci } \\
\text {-Transportation }\end{array}$ \\
\hline $\begin{array}{l}\text {-Esese and Cost of } \\
\text { Siting. Construction, } \\
\text { Operation. \& Closure }\end{array}$ & $\begin{array}{l}\text {-Surface characterist. } \\
\text { - Rock characteristics } \\
\text {-Hydrology }\end{array}$ \\
\hline
\end{tabular}

- Hydrology 
10 CFR 960 Subpart D

Preclosure Technical Guidelines Table

\begin{tabular}{|c|c|c|c|c|c|}
\hline & Teommionl Quldelline & Quallifying Conglition & Favorabile condition & Potentially Actoree Conditione & Disoually yino cendullone \\
\hline \multirow{4}{*}{ 递 } & 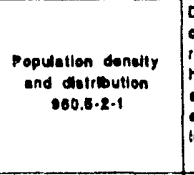 & 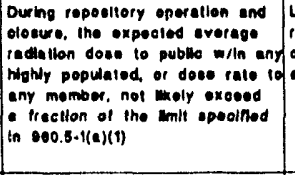 & 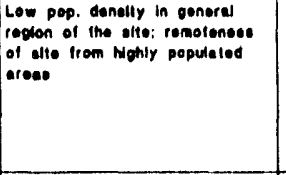 & 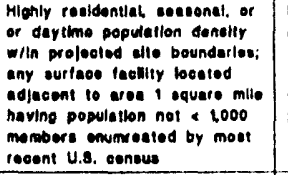 & 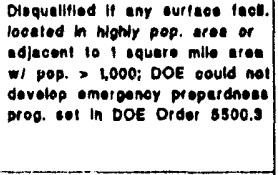 \\
\hline & $\begin{array}{l}\text { 8116. Ownorehlp } \\
\text { and Contul } \\
\text { Peo.s-2-2 }\end{array}$ & 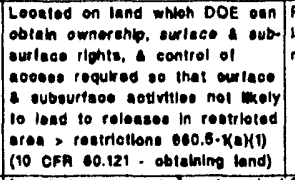 & 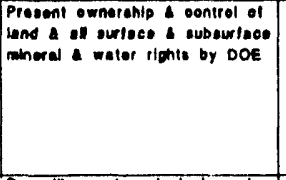 & 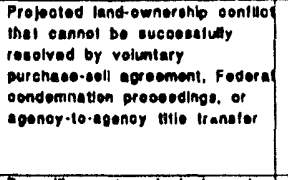 & nono \\
\hline & $\begin{array}{l}\text { Motorolegy } \\
\text { 200.5-2.3 }\end{array}$ & 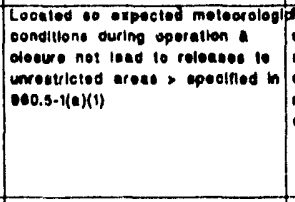 & 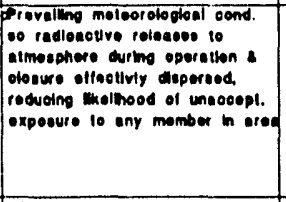 & 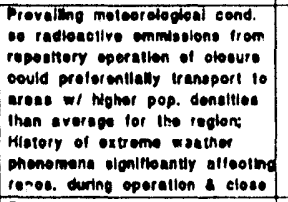 & none \\
\hline & 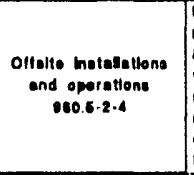 & 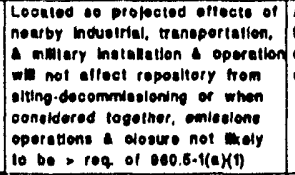 & 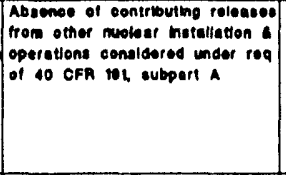 & 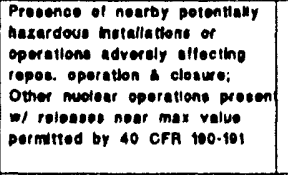 & 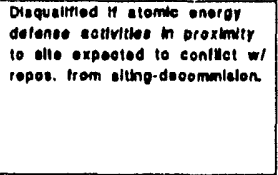 \\
\hline \multirow{3}{*}{ 圈 } & $\begin{array}{c}\text { Environmental qualliy } \\
\text { ve0,s.2.5 }\end{array}$ & 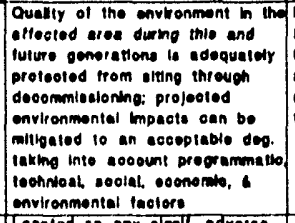 & 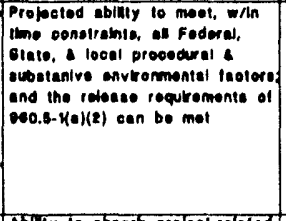 & 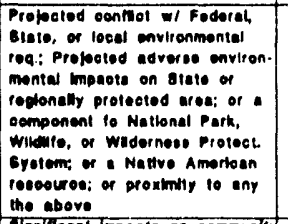 & 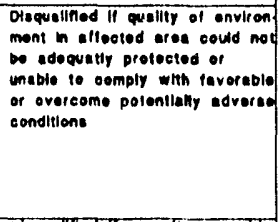 \\
\hline & 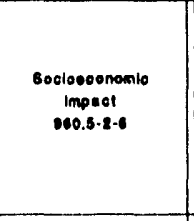 & 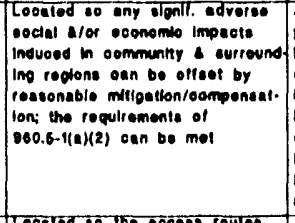 & 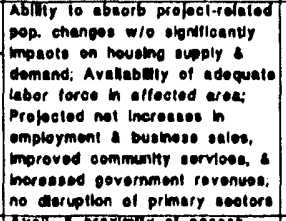 & 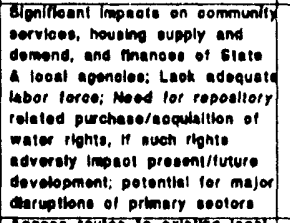 & 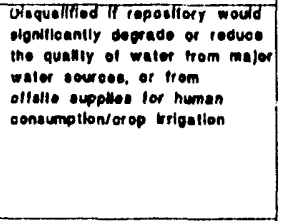 \\
\hline & $\begin{array}{l}\text { Tranoportation } \\
\text { poot.5-2-7 }\end{array}$ & 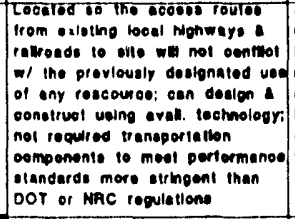 & 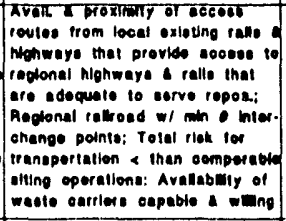 & 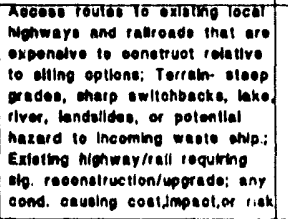 & none \\
\hline \multirow{2}{*}{ : } & $\begin{array}{c}\text { Buttoce Charnctorlsilled } \\
060.6-2 \cdot 0\end{array}$ & 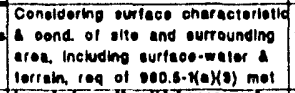 & $\begin{array}{l}\text { Qomorably iat, woll-dralnod } \\
\text { torrem }\end{array}$ & 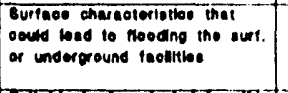 & none \\
\hline & $\begin{array}{c}\text { Roch Chareotortatios } \\
\text { Os0.6-2.0 }\end{array}$ & 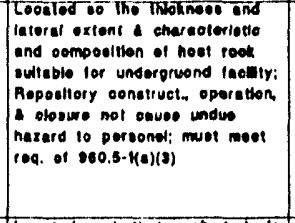 & 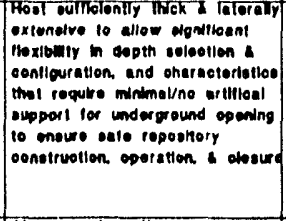 & 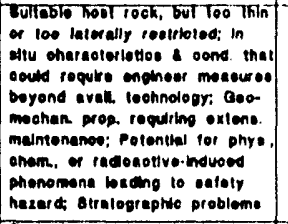 & 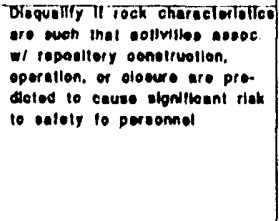 \\
\hline $\int_{0}^{5}$ & $\begin{array}{l}\text { Hydiotogy } \\
060.6-2 \cdot 10\end{array}$ & 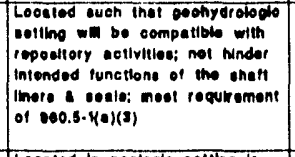 & 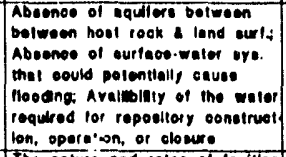 & 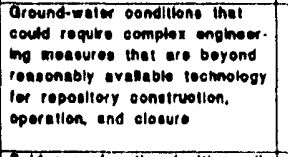 & 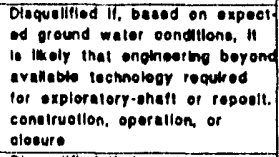 \\
\hline 8 & $\begin{array}{l}\text { Tootontos } \\
000.6 \cdot 2 \cdot 11\end{array}$ & 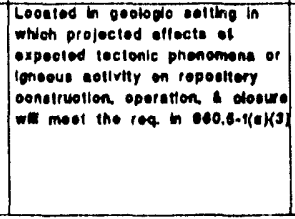 & 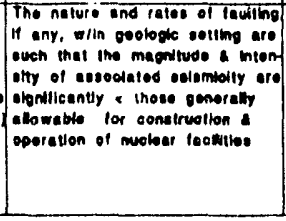 & 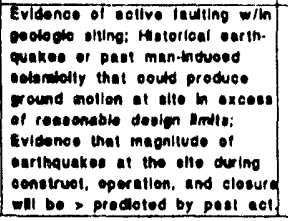 & 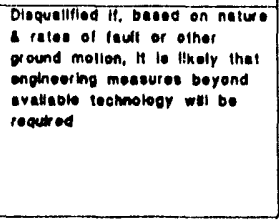 \\
\hline
\end{tabular}

NOTE: Adetionat miermation in al of theos aroes loosice in Apponatix iv. 


\section{CFr 191 Subpart B \\ Enviornmental Standards for the Uranium Fuel Cycle Compliance Structure}

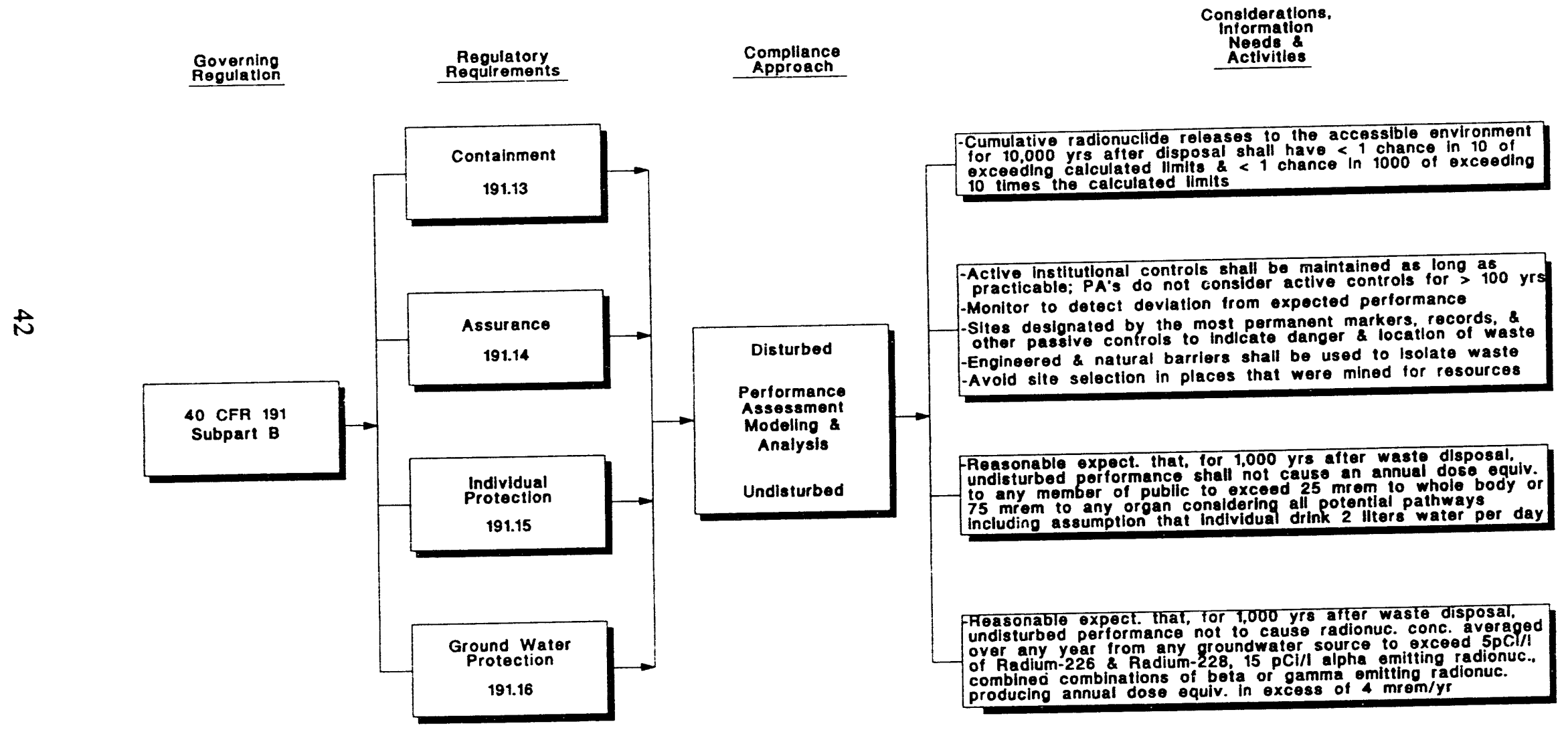




\section{CFR 264 Subpart $X$ \\ Permit for Misc. Unit Compliance Structure}

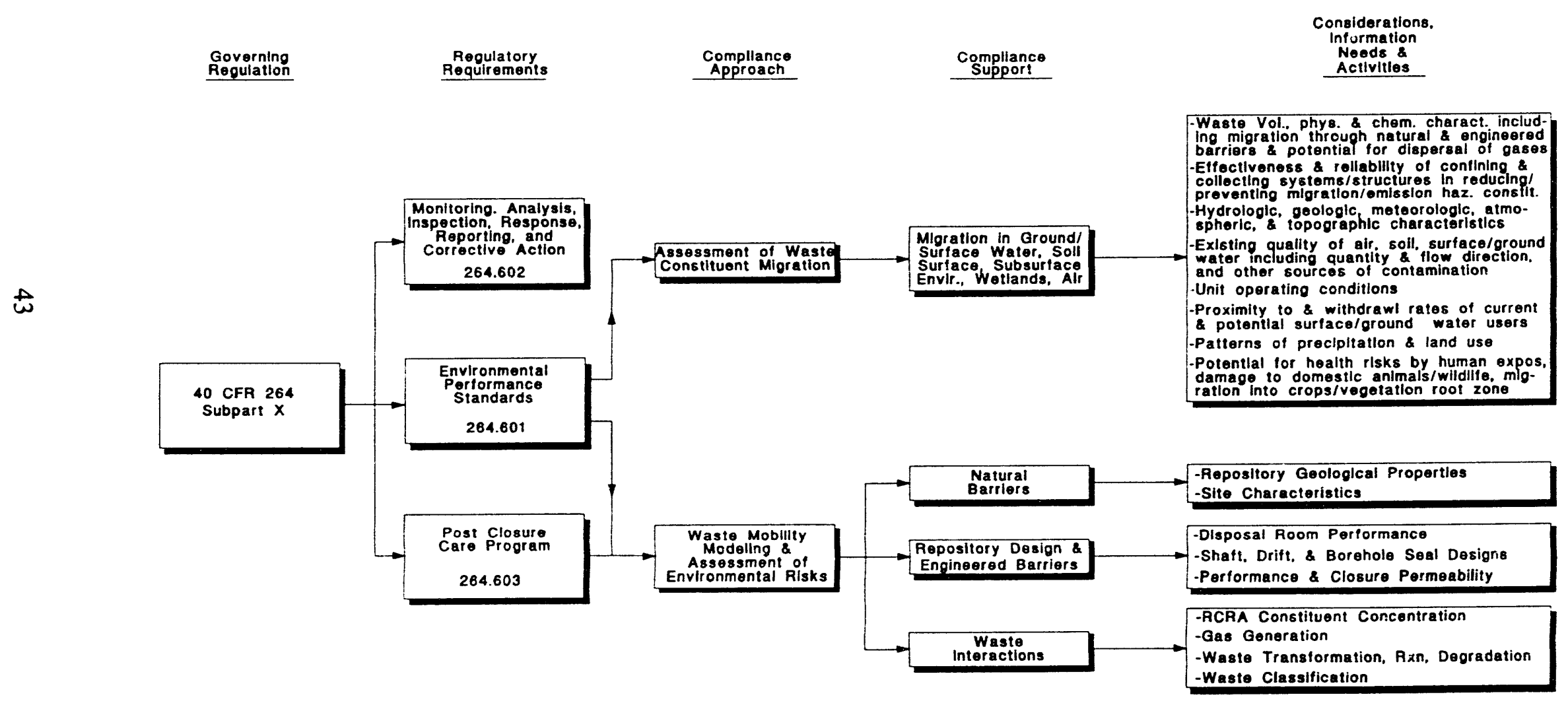




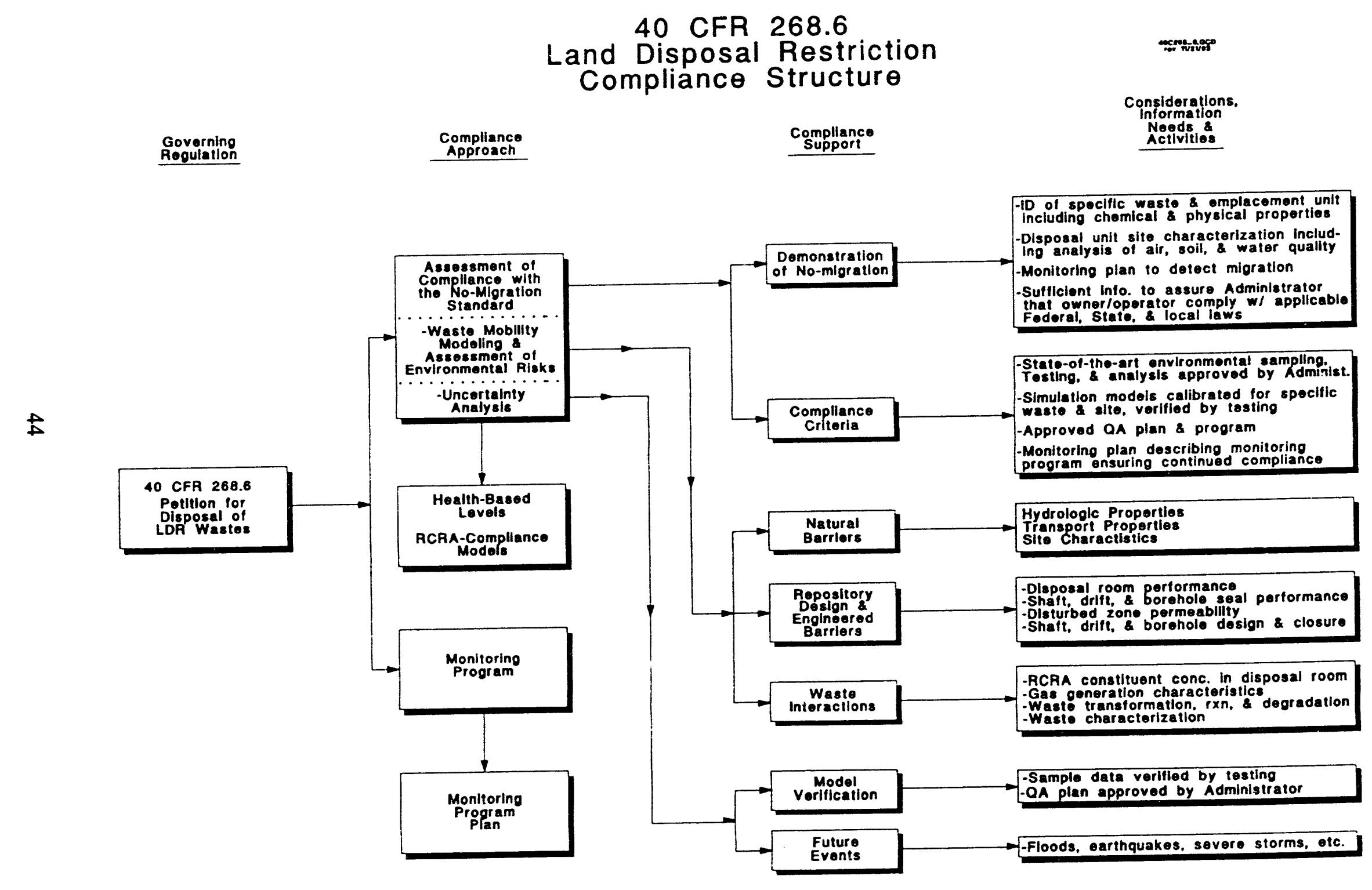




\section{CFR 20 Standards for Protection Against Radiation}

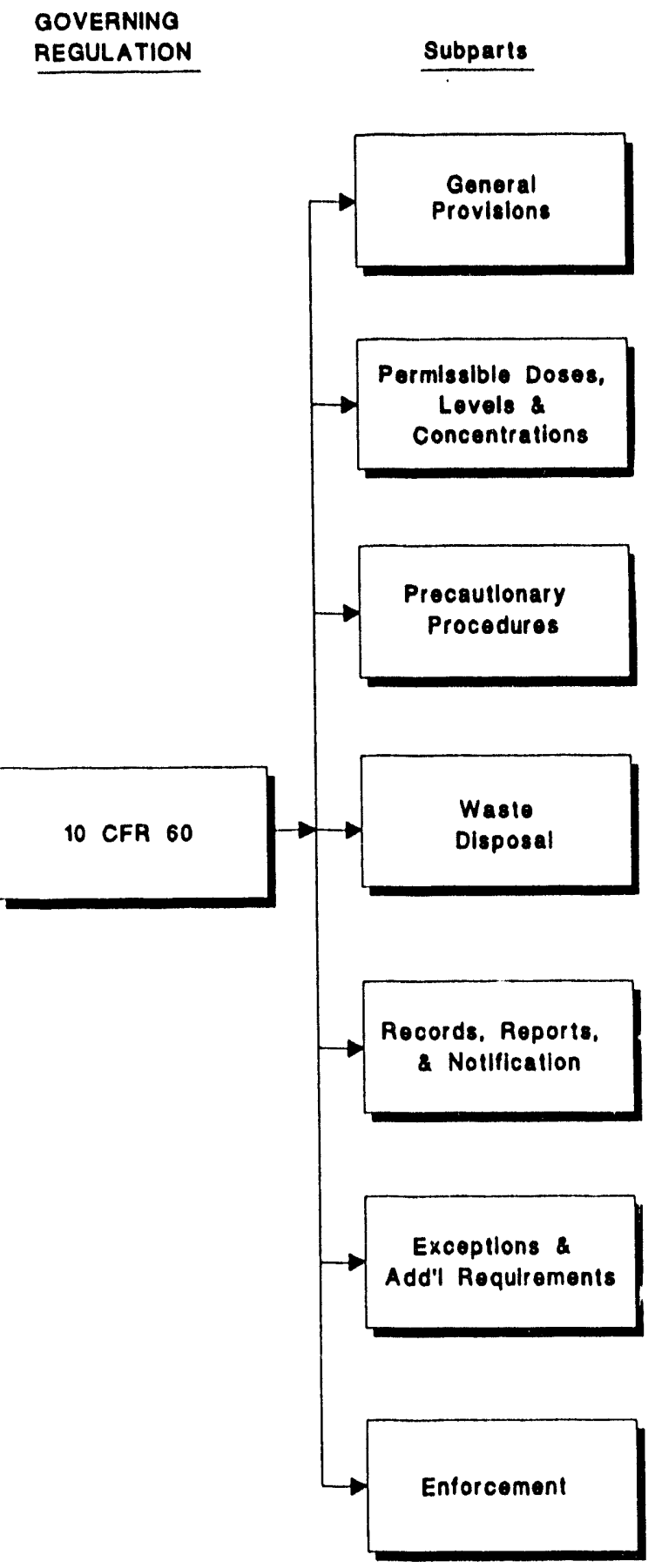




\section{DOE Order 5820.2A - Radioactive Waste Management: Chapter I - High Level Waste; Section 3d - Disposal Requirements

Governing

Information

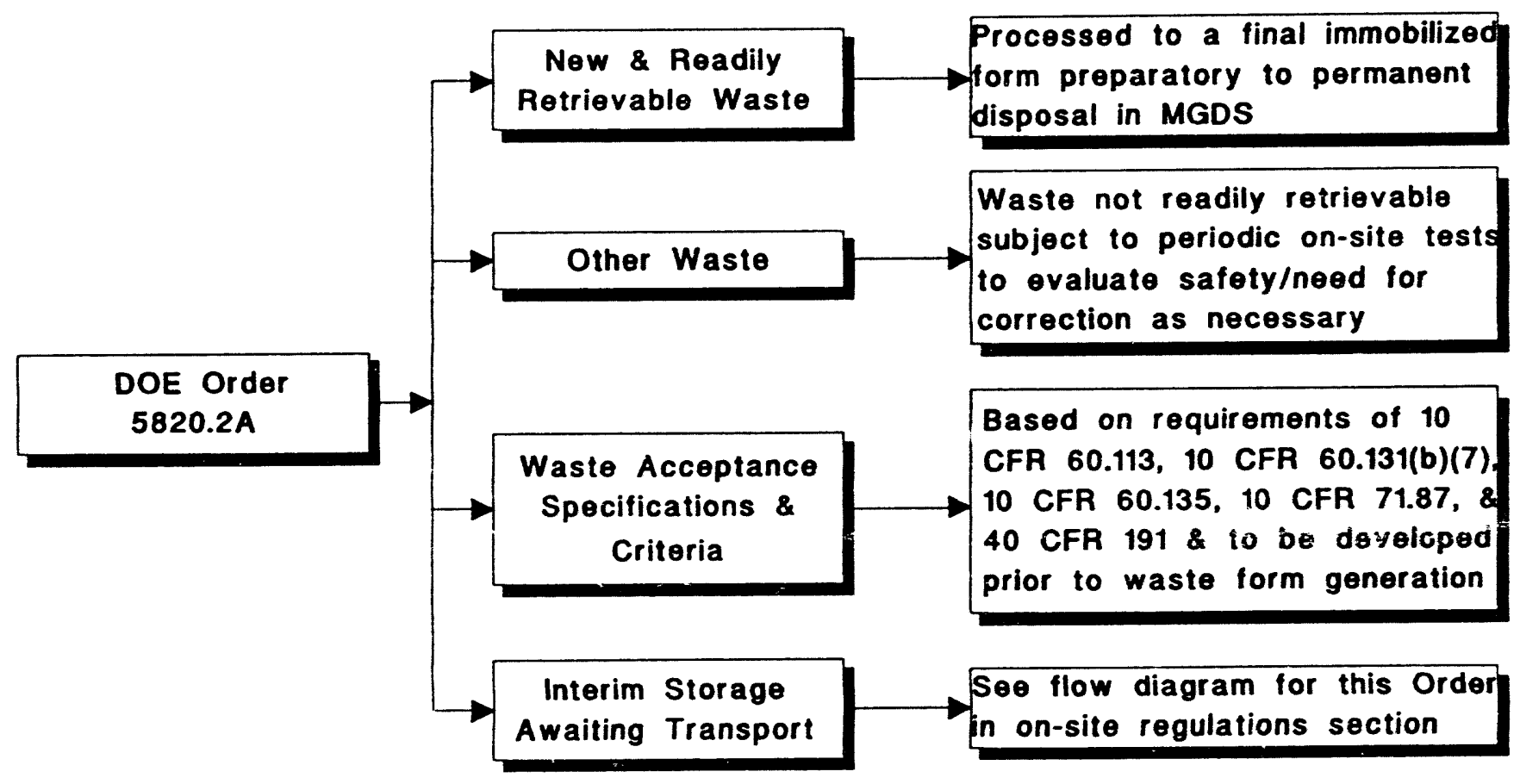




\section{ACRONYMS}

$\begin{array}{ll}\text { AEA } & \text { Atomic Energy Act } \\ \text { AEC } & \text { Atomic Energy Commission } \\ \text { ALARA } & \text { As Low As Reasonably Achievable } \\ \text { CFR } & \text { Code of Federal Regulations } \\ \text { COC } & \text { Certificate of Compliance } \\ \text { DOD } & \text { Department of Defense } \\ \text { DOE } & \text { Department of Energy } \\ \text { DOT } & \text { Department of Transportation } \\ \text { EIS } & \text { Environmental Impact Statement } \\ \text { EPA } & \text { U.S. Environmental Protection Agency } \\ \text { ERDA } & \text { Energy Research and Development Administration } \\ \text { HLW } & \text { High-level Waste } \\ \text { INEL } & \text { Idaho National Engineering Laboratory } \\ \text { MGDS } & \text { Mined Geologic Disposal System } \\ \text { NEPA } & \text { National Environmental Policy Act } \\ \text { NRC } & \text { U.S. Nuclear Regulatory Commission } \\ \text { NWPA } & \text { Nuclear Waste Policy Act } \\ \text { PA } & \text { Performance Assessment } \\ \text { QA } & \text { Quality Assurance } \\ \text { RCRA } & \text { Resource Conservation and Recovery Act } \\ \text { SAR } & \text { Safety Analysis Report } \\ \text { SNF } & \text { Spent Nuclear Fuel } \\ \text { TRU } & \text { Transuranic Waste }\end{array}$




\section{INDEX OF DIRECTIVES}

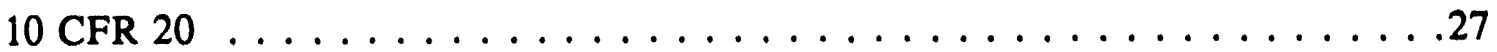

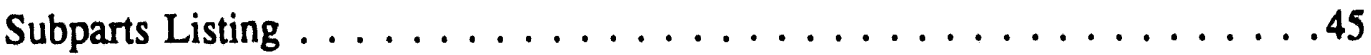

10 CFR 60

Subpart B

30-32

Subpart E

33-35

Subparts Listing . . . . . . . . . . . . . . . . . 29

10 CFR 71

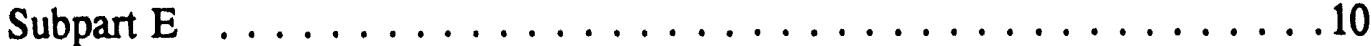

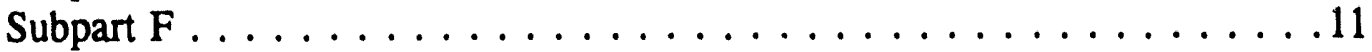

Subparts Listing $\ldots \ldots \ldots \ldots \ldots \ldots \ldots \ldots \ldots \ldots$

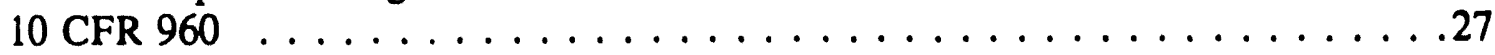

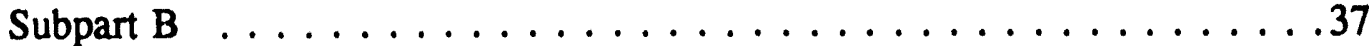

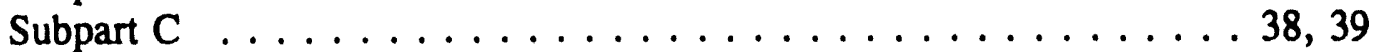

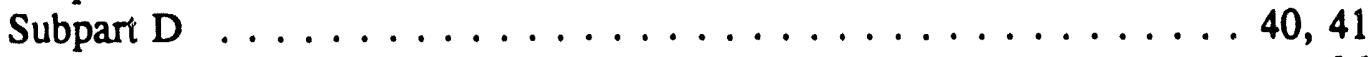

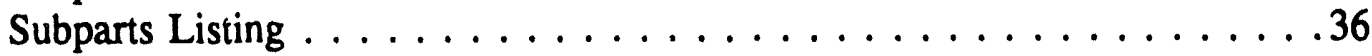

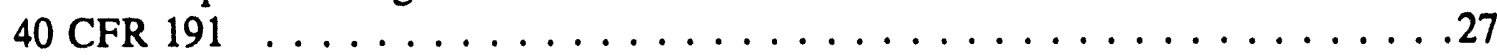

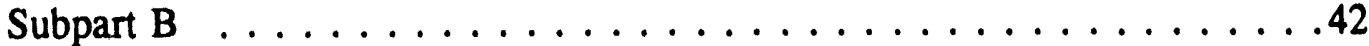

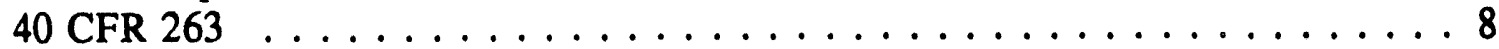

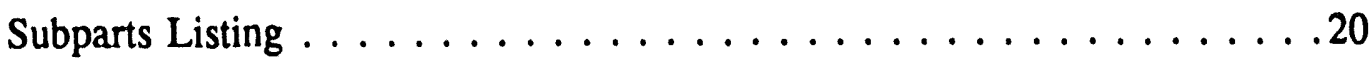

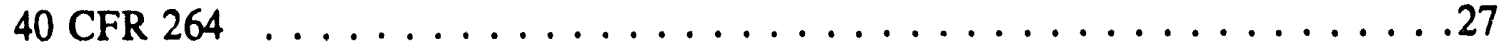

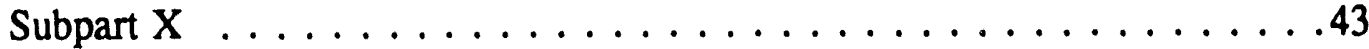

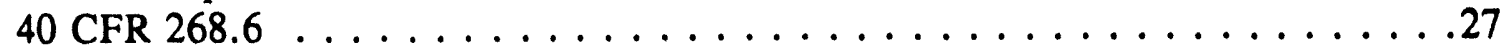

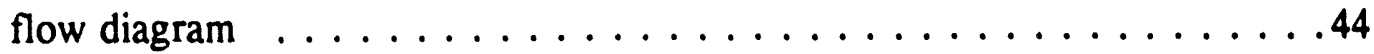

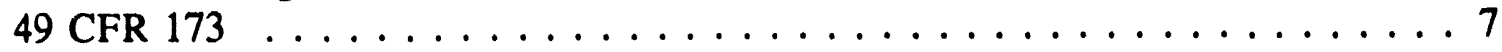

Subpart I . . . . . . . . . . . . . . . . . 14-18

Subpart I flow diagram listing $\ldots \ldots \ldots \ldots \ldots \ldots \ldots \ldots \ldots \ldots$

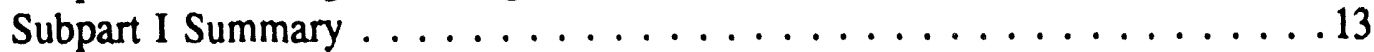

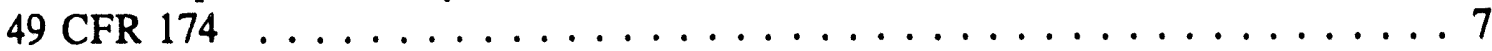

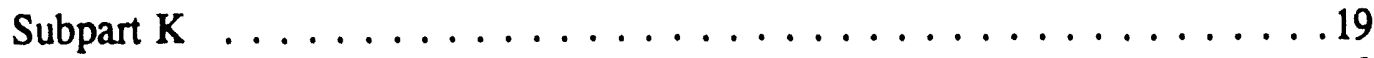

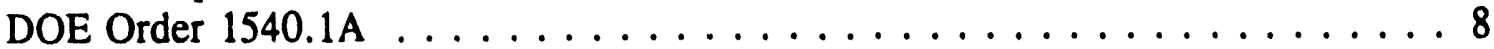

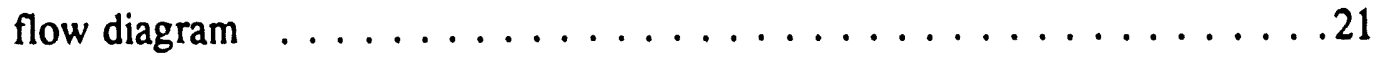

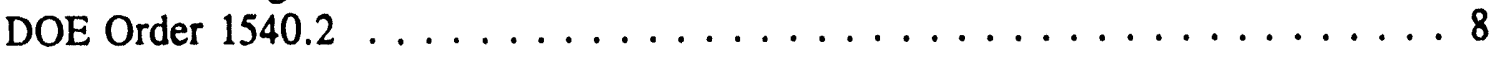

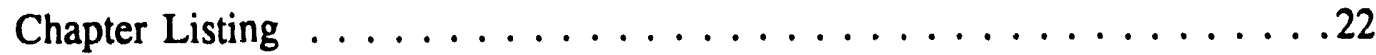

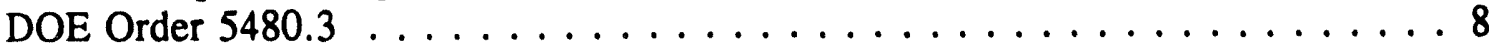

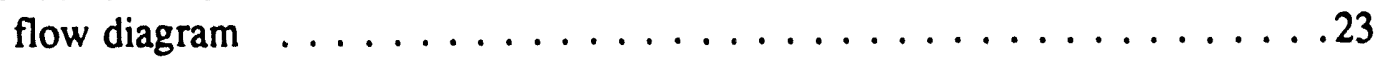

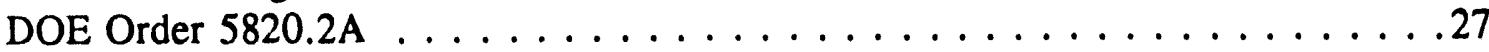

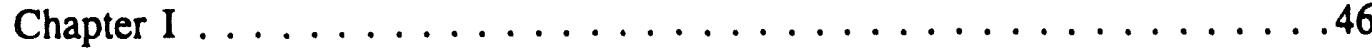

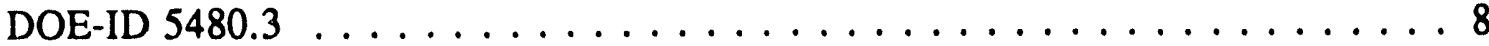

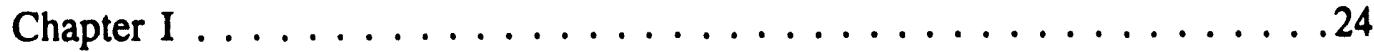

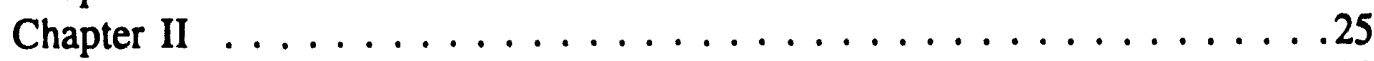

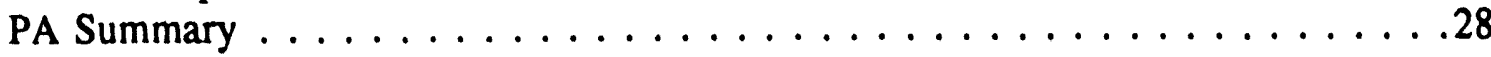




\section{REFERENCES}

DOE/ID-10405, Idaho National Engineering Laboratory Installation Roadmap Document, DRAFT, May 1993.

ORNL/M-1717, Hazardous Materials Transportation Act, Revision 5, October 15, 1991, Oak Ridge National Laboratory.

ORNL/M-1900, Atomic Energy Act and Related Legislation, Revision 6, September 1, 1992, Oak Ridge National Laboratory.

SAND86-0121, Assessing Compliance With the EPA High-Levei Waste Standard: An Overview, October 1986, Sandia National Laboratories.

Training Seminar Manual, Environmental Regulations Overview, July 1992, WINCO Environmental Training Personnel. 


\section{APPENDIX A}

This Appendix provides potentially supplemental information for ensuring compliance with the requirements summarized in this document. It contains two letters reporting the results of an investigation of criticality issues related to the direct disposal of fuel in a geologic repository conducted by R.L. Nebeker, of WINCO.

Attachments to the letters outline and summarize various NRC Regulatory guides and ANSI standards. These summaries provide additional information relating to criticality safety. Attachment 1 of Neb-03-91 and Attachment 3 of Neb-04-91 summarize the content of various Regulatory Guides, ANSI Standards, and Regulations; Attachment 2 of Neb-03-91 gives impacts, proposed resolutions, and long term implications of specific requirements set forth in 10 CFR 60, 40 CFR 191, and DOE Order 5480.5. These two regulations, 10 CFR 60 and 40 CFR 191, have been summarized in more detail in the MGDS section. Attachment 2 of Neb-04-91 does the same for various Regulatory guides; however, no flow diagram summaries for Regulatory guides are prepared in this document.

Other potentially applicable federal directives are contained in DOE Order 5480.4 Environmental Protection, Safety, and Health Protection Standards. This Order categorically lists directives and reported procedures to be followed when conducting activities outlined in the various sections of this document. For example, under the Radioactive Material Packaging and Transportation Safety section (Page 12 of Attachment 3 of this Order), references such as ORNL-NSIC-68, 1970, "Cask Designers Guide" (ORNL) and NRC Guide 7.4, "Leakage Tests for Shipment of Radioactive Materials" (NRC) are listed.

No detailed 'jvestigation into the applicability of the references in this appendix has been conducted. However, it is believed that some of these referrals will contain useful information as new operations and activities at the INEL relative to the SF\&WMTDP are developed. 


\section{INE}

io National Engineoring L.sboratory

Neb-03-91

Fiom R. L. Nebeker

Phone . 6-3578

Dale : October 22, 1991

Subiecl: Criticality Implications of Direct Disposal of Fuel

Io N. A. Chipman, Fellow Engineer

New Business and Special Studies
CC:
G. B. Frandsen
R. N. Henry
G. W. Hogg

V. C. Maio

B. R. Wheeler

R. L. Nebeker - 2

As you requested, I have reviewed various regulations and standards concerning criticality issues $r \leq l$ ated to the direct disposal of fuel in a geological repository. A list of these regulations and their highlights are provided in Attachment 1 .

In general, the regulations do not specifically apply to repository disposition, but to other aspects of fuel handling. However, regulations for repository disposition of fuel would be expected to contain the general restrictions shown in these regulations.

Attachment 2 summarizes the requirements of these regulations as well as other regulations concerning fuel disposition in a repository (mainly concerning the stability of the repository package) which indirectly impact criticality control.

For direct disposal of fuel, two time periods are of concern: "short," corresponding roughly to the length of time a canister must retain its integrity and "long," when container integrity cannot be guaranteed. The need for criticality control in a repository is discussed in 10CFR60.131(7), but nothing is said regarding the time periods for which the criticality control requirements must be met. The NRC staff has identified this as an "uncertainty", and the issue is presently being studied by NRC personnel.

Rick Weller of the High-Level Waste Program for the NRC was contacted regarding the time periods for which criticality control requirements in 10CFR60 must be met. He indicated that a staff position is being developed and an NRC Guidance Document on the subject will be issued in the next several months. Most likely, the guidance will require that criticality control be considered in both the preclosure and post closure periods. Analyses must be completed to evaluate criticality impacts in meeting performance criteria for the duration of the period of interest, which is assumed to be 10,000 years, based on EPA requirements in 40CFR191. 
N. A. Chipman

Page 2

Neb-03-91

October 22, 1991

While it appears that criticality could be controlled with adequate design in the "short" time period, it also appears that there is absolutely no way to guarantee that a criticality will not occur over the time the fissile material in the spent fuel would remain in the repository.

Obviousiy, disposing of the fuel directly instead of removing the fissile material (uranium and plutonium) increases the care and attention that must be given to the waste package because of criticality concerns. Some of the additional concerns are:

1. Each "package" must be assured to be critically safe. For HEU fuel, this could invol"e additional fuel disassembly steps prior to packaging or adding nuclea? poisons to the package.

2. The spacing within the repository would have to be analyzed to determine that the array of spent fuel is subcritical for all possibilities. This spacing may be mcre or less restrictive than spacing for heat removal.

3. The intrusion of water into the repository over long time periods would adversely affect the fuel reactivity and would have to be considered in all criticality analyses.

4. Over long periods, movements through faulting or other earth shifting methods might change the geometry of the fuel array or damage the package and reduce its $l$ ifetime, making it more reactive.

5. Over long periods, nuclear poisons could conceivably be selectively leached from the fuel package, resulting in higher reactivities. The decay of fission product poisons in the fuel would also increase the reactivity in the fuel, even in the "short" term.

6. Selective movement of uranium is conceivable over the long term if the uranium is dissolved and reprecipitated. This also might increase the reactivity wichin the repository.

7. Typically, inspections are required to verify that any nuclear poisons used for criticality control remain in place and are not removed. Inspection to assure canister arrays and the integrity of the canister may also be an issue. Inspection after placement in a repository might be impossible or would require extensive fuel handling.

8. Intrusion to prevent diversion of $\mathrm{HEU}$ is not addressed in existing regulations but would be a logical safeguard issue that would be expected to arise during the public review process. Unlike commercial fuel, HEU fuel would require less refining to prevent an SNM threat. 
N. A. Chipman

Page 3

$\mathrm{Neb}-03-01$

October 22, 1991

In summary, it would appear that confinement of fission products via engineered barriers (canister and repository) is feasible, but assurance of criticality safety cannot be demonstrated for geologic time.

In addition to these criticality issues there are other issues concerning fuel configuration that indirectly affect criticality control that must be resolved if fuel is disposed of directly. Among these are:

1. Elimination of combustibles/reactives will be required of fuels containing graphite or carbides before they can meet today's requirements. This could require extensive pretreatment prior to disposal.

2. Solidification of particulate matter would be required for any powdered fuel or fuel residue, again requiring a type of pretreatment.

3. Fuels would have to be characterized to demonstrate their ability to retain fission products and to assure regulators that material going to the repository is known and understood. Because of the many types of fuel (over 30 general types at the ICPP alone), this would require an extensive amount of time and money (probably over a billion dollars over many years).

It is doubtful that these issues can be analyzed in a manner that would convince the public that HEU type fuel or even commercial LEU fuel can be disposed of directly to a repository without generating a criticality safety risk. Whether or not the issues are really problems may be immaterial; if they are perceived as problems by the public, it will be impossible or difficult to get public acceptance. While it may be technically acceptable to say that the risk of a criticality deep underground in a geological repository is acceptable and can be analyzed based on data from the Oklo Natural Reactor, it is doubtful that the general public would accept such a risk. Furthermore, failure to remove the fissile material (the technology does exist) casts doubt on DOE's commitment to make nuclear safety their number one priority.

Obviously, this issue will have to be developed further over a period of time. If I can be of any further help, or supply further information, let me know.

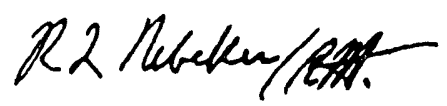

R. L. Nebekèr

Fellow Engineer

Attachments 


\section{Attachment 1}

Summary of Guidance Documents Pertaining to Criticality and Fuel Storage

\begin{tabular}{|l|l|l|}
\hline Document & Title & Summary of Contents \\
\hline 10CFR60 & $\begin{array}{l}\text { Disposal of High-Level } \\
\text { Radioactive Wastes in } \\
\text { Geologic Repositories }\end{array}$ & $\begin{array}{l}\text { This regulation lists general provisions for disposal of } \\
\text { HLW and describes license application requirements. It } \\
\text { Contains performance objectives and design criteria. }\end{array}$ \\
\hline 10CFR60.113 & $\begin{array}{l}\text { Performance of Particular } \\
\text { Barriers After Permanent } \\
\text { Closure }\end{array}$ & $\begin{array}{l}\text { Requires HLW containment during fission product decay time. } \\
\text { Requires gradual release of radionuclides from barrier } \\
\text { system. Requires containment for 300-1000 years. }\end{array}$ \\
\hline 10CFR60.131 & $\begin{array}{l}\text { General Design Criteria } \\
\text { for the Geologic } \\
\text { Repository Operations Area }\end{array}$ & $\begin{array}{l}\text { Requires radiation doses, levels and concentrations to be } \\
\text { within established levels. Lists design criteria for } \\
\text { repository. Requires steps to minimize possibility of a } \\
\text { criticality. }\end{array}$ \\
\hline 10CFR60.135 & $\begin{array}{l}\text { Criteria for the Waste } \\
\text { Package and Its Components }\end{array}$ & $\begin{array}{l}\text { Requires package for HLW to be able to withstand } \\
\text { environmental attacks. Lists criteria to be met by waste } \\
\text { forms. }\end{array}$ \\
\hline 40 CFR191 & $\begin{array}{l}\text { Environmental Radiation } \\
\text { Protection Standards for } \\
\text { Management and Disposal of } \\
\text { Spent Nuclear Fuel, High- } \\
\text { Level and Transuranic } \\
\text { Radioactive Waste }\end{array}$ & $\begin{array}{l}\text { Lists standards for radiation doses received by public as a } \\
\text { result of management and disposal activities. } \\
\text { radioactive material releases as a result of disposal. }\end{array}$ \\
\hline 40 CFR191.03 & $\begin{array}{l}\text { Standards } \\
\text { Containment Requirements }\end{array}$ & $\begin{array}{l}\text { Provides standards for design of containment and } 1 \text { ists } \\
\text { release likelihoods to be obtained. }\end{array}$ \\
\hline
\end{tabular}




\begin{tabular}{|c|c|c|}
\hline Document & Title & Summary of Contents \\
\hline 40CFR191.14 & Assurance Requirements & $\begin{array}{l}\text { Discusses institutional controls, monitoring, } \\
\text { marking, barriers, mining, and waste removal. }\end{array}$ \\
\hline 40CFR191 Appendix A & Table for Subpart 13 & $\begin{array}{l}\text { Lists cumulative releases to the accessible } \\
\text { environment for } 10,000 \text { years after disposal for } \\
\text { specific nuclides and groups. }\end{array}$ \\
\hline DOE Order 5480.5 & $\begin{array}{l}\text { Safety of Nuclear } \\
\text { Facilities }\end{array}$ & $\begin{array}{l}\text { Describes basic requirements for } U O E^{\prime} s \\
\text { environment, safety and health program. Basic } \\
\text { elements of nuclear criticality safety are } \\
\text { presented along with Nuclear critical ty safety } \\
\text { control parameters. }\end{array}$ \\
\hline NRC Regulatory Guide 1.13 & $\begin{array}{l}\text { Spent Fuel Storage } \\
\text { Facility Design Basis }\end{array}$ & $\begin{array}{l}\text { Presents methods for assuring that fuel storage } \\
\text { and handling system are designed for adequate } \\
\text { safety under normal and postulated accident } \\
\text { conditions. }\end{array}$ \\
\hline NRC Regulatory Guide 3.4 & $\begin{array}{l}\text { Nuclear Criticality Safety } \\
\text { in Operations with } \\
\text { Fissionable Materials at } \\
\text { Fuels and Materials } \\
\text { Facilities }\end{array}$ & $\begin{array}{l}\text { Describes procedures for preventing accidental } \\
\text { criticality in operations with fissionable } \\
\text { materials at fuel cycle facilities other than } \\
\text { reactors. Refers to ANSI/ANS-8.1-1983. }\end{array}$ \\
\hline NRC Regulatory Guide 3.33 & $\begin{array}{l}\text { Assumptions used for } \\
\text { Evaluating the Potential } \\
\text { Radiological Consequences } \\
\text { of Accidental Nuclear } \\
\text { Criticality in a Fuel } \\
\text { Reprocessing Plant } \\
\end{array}$ & $\begin{array}{l}\text { Provides methods and equations for determining } \\
\text { the impacts of a criticality on the public } \\
\text { health and safety. Describes how to estimate } \\
\text { the consequences of an accidental criticality as } \\
\text { it applies to a fuel reprocessing plant. }\end{array}$ \\
\hline NRC Regulatory Guide 3.34 & $\begin{array}{l}\text { Assumptions used for } \\
\text { Evaluating the Potential } \\
\text { Radiological Consequences } \\
\text { of Accidental Nuclear } \\
\text { Criticality in a Uranium } \\
\text { Fuel Fabrication Plant }\end{array}$ & $\begin{array}{l}\text { Provides methods for determining the impacts of } \\
\text { a criticality on the public health and safety as } \\
\text { it applies to a uranium fuel fabrication plant. }\end{array}$ \\
\hline
\end{tabular}




\begin{tabular}{|c|c|c|}
\hline Document & Title & Summi:y of Contents \\
\hline $\begin{array}{l}\text { NRC Regulatory Guide } \\
3.43\end{array}$ & $\begin{array}{l}\text { Nuclear Criticality } \\
\text { Safety in the Storage of } \\
\text { Fissile Materials }\end{array}$ & $\begin{array}{l}\text { Provides guidance for procedures to avoid accidental } \\
\text { criticality during storage of fissile materials. } \\
\text { Refers to ANSI N16.5-1975. }\end{array}$ \\
\hline $\begin{array}{l}\text { NRC Regulatory Guide } \\
3.57\end{array}$ & $\begin{array}{l}\text { Administrative Practices } \\
\text { for Nuclear Criticality } \\
\text { Safety at Fuels and } \\
\text { Materials Facilities }\end{array}$ & $\begin{array}{l}\text { Provides guidance for safety procedures which are } \\
\text { part of a nuclear safety program. Refers to ANSI/ANS } \\
8.19-1984 \text {. }\end{array}$ \\
\hline $\begin{array}{l}\text { NRC Regulatory Guide } \\
3.58\end{array}$ & $\begin{array}{l}\text { Critical Safety for } \\
\text { Handling, Storing, and } \\
\text { Transporting LWR Fuel at } \\
\text { Fuels and Materials } \\
\text { Facilities }\end{array}$ & $\begin{array}{l}\text { Provides guidance for preventing criticality } \\
\text { accidents during handling, storage, and transporting } \\
\text { of LWR fuel outside of nuclear reactors. Refers to } \\
\text { ANSI/ANS-8.17-1984. }\end{array}$ \\
\hline $\begin{array}{l}\text { NRC Regulatory Guide } \\
3.60\end{array}$ & $\begin{array}{l}\text { Design of an Independent } \\
\text { Spent Fuel Storage } \\
\text { Installation (Dry } \\
\text { Storage) }\end{array}$ & $\begin{array}{l}\text { This document provide guidance for use in the design } \\
\text { of a dry storage independent spent fuel storage } \\
\text { installation. It refers to ANSI/ANS-57.9-1984. }\end{array}$ \\
\hline $\begin{array}{l}\text { NRC Regulatory Guide } \\
8.12\end{array}$ & $\begin{array}{l}\text { Criticality Accident } \\
\text { Alarm Systems }\end{array}$ & $\begin{array}{l}\text { This guide describes a system for meeting } \\
\text { requirements for a criticality accident alarm system. } \\
\text { it refers to ANSI/ANS-8.3-1986. }\end{array}$ \\
\hline ANSI/ANS-8.1-1983 & $\begin{array}{l}\text { American National } \\
\text { Standard for Nuclear } \\
\text { Critical ity Safety in } \\
\text { Operations with } \\
\text { Fissionable Materials } \\
\text { Outside Reactors } \\
\end{array}$ & $\begin{array}{l}\text { This standard provides guidance for the prevention of } \\
\text { criticality accidents in the handling, storing, } \\
\text { processing, and transporting of fissionable material. }\end{array}$ \\
\hline ANSI/ANS-8.3-1986 & $\begin{array}{l}\text { Criticality Accident } \\
\text { Alarm System }\end{array}$ & $\begin{array}{l}\text { This standard provides guidance for the establishment } \\
\text { and maintenance of an alarm system to initiate } \\
\text { personnel evacuation in the event of inadvertent } \\
\text { criticality. }\end{array}$ \\
\hline
\end{tabular}




\begin{tabular}{|l|l|l|}
\hline Document & Title & Summary of Contents \\
\hline ANS-8.7/ANSI N16.5-1975 & $\begin{array}{l}\text { Guide for Nuclear } \\
\text { Criticality Safety in } \\
\text { the Storage of Fissile } \\
\text { Materials }\end{array}$ & $\begin{array}{l}\text { This guide provides orientation and direction to } \\
\text { nuclear safety practices. }\end{array}$ \\
\hline ANSI/ANS-8.17-1984 & $\begin{array}{l}\text { American National } \\
\text { Standard, Criticality } \\
\text { Safety Criteria for the } \\
\text { Handling, Storage, and } \\
\text { Transportation of LWR } \\
\text { Fuel Outside Reactors }\end{array}$ & $\begin{array}{l}\text { This standard provides basic requirements that } \\
\text { address the criticality safety aspects of a facility } \\
\text { conjunction with other safety standards or } \\
\text { regulations. }\end{array}$ \\
\hline ANSI/ANS 8.19-1984 & $\begin{array}{l}\text { American National } \\
\text { Standard - } \\
\text { Administrative Practices } \\
\text { for Nuclear Criticality } \\
\text { Safety }\end{array}$ & $\begin{array}{l}\text { This standard presents a codification of certain } \\
\text { common safety elements related to criticality }\end{array}$ \\
\hline ANSI/ANS-5.79-1984 & $\begin{array}{l}\text { American National } \\
\text { Standard - Design } \\
\text { Criteria for an } \\
\text { Independent Spent Fuel } \\
\text { Storage Installation } \\
\text { (Dry Storage Type) }\end{array}$ & $\begin{array}{l}\text { This standard provides design criteria for an } \\
\text { spent fuel which incorporates one or more of the dry } \\
\text { storage concepts. }\end{array}$ \\
\hline \hline
\end{tabular}


Attachment 2

Criticality and Other Requirements for Spent Fuel Disposal

\begin{tabular}{|c|c|c|c|c|}
\hline $\begin{array}{l}\text { Assumption/ } \\
\text { Requirements }\end{array}$ & Reference & Impact & $\begin{array}{l}\text { Proposed } \\
\text { Resolution } \\
\end{array}$ & $\begin{array}{l}\text { Comments/Long Term } \\
\text { Implications }\end{array}$ \\
\hline $\begin{array}{l}\text { Containment until } \\
\text { fission products decay } \\
\text { ( } 300-1000 \text { years) }\end{array}$ & 10CFR60.113 & $\begin{array}{l}\text { Extensive fuel } \\
\text { characterization } \\
\text { required for many } \\
\text { different fuel } \\
\text { types. }\end{array}$ & $\begin{array}{l}\text { Repository to be } \\
\text { constructed to } \\
\text { provide } \\
\text { containment: } \\
\text { characterize } \\
\text { fuel. } \\
\end{array}$ & $\begin{array}{l}\text { Probably attainable } \\
\text { for required time. }\end{array}$ \\
\hline $\begin{array}{l}\text { Slow release of } \\
\text { radionuclides } \\
(1 / 100,000 \text { per year })\end{array}$ & 10CFR60.113 & $\begin{array}{l}\text { Must demonstrate for } \\
\text { fuel inventory as } \\
\text { well as criticality } \\
\text { fission products. } \\
\text { Must show slow } \\
\text { release of gaseous } \\
\text { fission products } \\
\text { from criticality. }\end{array}$ & $\begin{array}{l}\text { Characterize } \\
\text { fuel, perform } \\
\text { analyses. }\end{array}$ & $\begin{array}{l}\text { Probably obtainable } \\
\text { for many fuels. }\end{array}$ \\
\hline $\begin{array}{l}\text { Maintain air } \\
\text { concentrations below } \\
10 \text { CFR } 20 \text { limits }\end{array}$ & 10CFR60.131 & $\begin{array}{l}\text { Retention of fission } \\
\text { products required. }\end{array}$ & $\begin{array}{l}\text { Canister should } \\
\text { be adequate if it } \\
\text { remains intact. }\end{array}$ & $\begin{array}{l}\text { Release of gaseous } \\
\text { fission products from } \\
\text { criticality must be } \\
\text { analyzed. }\end{array}$ \\
\hline $\begin{array}{l}\text { Double contingency to } \\
\text { prevent criticality }\end{array}$ & $\begin{array}{l}\text { 10CRF60.131(7) } \\
\text { DOE } 5480.5, \\
\text { ANSI/ANS-8.1- } \\
1983\end{array}$ & $\begin{array}{l}\text { May require } \\
\text { extensive spacing, } \\
\text { use of poison, or } \\
\text { other requirements. }\end{array}$ & $\begin{array}{l}\text { Various solutions } \\
\text { possible for } \\
\text { short term. }\end{array}$ & $\begin{array}{l}\text { Unable to demonstrate } \\
\text { compliance in long } \\
\text { term. }\end{array}$ \\
\hline $\begin{array}{l}\text { Keff to have a } 5 \% \\
\text { margin }\end{array}$ & 10 CFR60.131(7) & $\begin{array}{l}\text { May limit spacing/ } \\
\text { packaging of fuel. }\end{array}$ & $\begin{array}{l}\text { Spacing, poisons, } \\
\text { disassembly or } \\
\text { other methods. }\end{array}$ & $\begin{array}{l}\text { Unable to demonstrate } \\
\text { compliance in long } \\
\text { term. }\end{array}$ \\
\hline
\end{tabular}




\begin{tabular}{|c|c|c|c|c|}
\hline $\begin{array}{l}\text { Assumption/ } \\
\text { Requirements }\end{array}$ & Reference & Impact & Proposed Resolution & $\begin{array}{l}\text { Comments/Long Term } \\
\text { Implications }\end{array}$ \\
\hline $\begin{array}{l}\text { In-situ chemical } \\
\text { reactions do not } \\
\text { compromise package }\end{array}$ & $\begin{array}{l}\text { 10CFR60. } \\
135\end{array}$ & $\begin{array}{l}\text { Must show that poisons } \\
\text { remain in place; that } \\
\text { uranium is not } \\
\text { concentrated by chemical } \\
\text { action. }\end{array}$ & $\begin{array}{l}\text { Canister will provide } \\
\text { protection during } \\
\text { "short" term. }\end{array}$ & $\begin{array}{l}\text { Long term } \\
\text { compliance will be } \\
\text { difficult to } \\
\text { demonstrate. }\end{array}$ \\
\hline $\begin{array}{l}\text { In-situ physical } \\
\text { properties do not } \\
\text { compromise package }\end{array}$ & $\begin{array}{l}10 \text { CFR } 60 \\
135\end{array}$ & $\begin{array}{l}\text { Physical movement of } \\
\text { fuel leading to } \\
\text { criticality must be } \\
\text { prevented. }\end{array}$ & $\begin{array}{l}\text { Repository geology will } \\
\text { demonstrate short-term } \\
\text { compliance. }\end{array}$ & $\begin{array}{l}\text { Long-term } \\
\text { stability will be } \\
\text { difficult to } \\
\text { demonstrate. }\end{array}$ \\
\hline $\begin{array}{l}\text { In-situ nuclear } \\
\text { properties do not } \\
\text { compromise package }\end{array}$ & $\begin{array}{l}10 \text { CFR } 60 . \\
135\end{array}$ & $\begin{array}{l}\text { High radiation, } \\
\text { criticality must not } \\
\text { impact package. }\end{array}$ & $\begin{array}{l}\text { Short term compliance } \\
\text { by spacing, poisons, } \\
\text { etc. }\end{array}$ & $\begin{array}{l}\text { Long-term } \\
\text { demonstration of } \\
\text { lack of damage } \\
\text { from criticality } \\
\text { difficult to } \\
\text { demonstrate. }\end{array}$ \\
\hline Consider solubility & $\begin{array}{l}10 C R F 60 . \\
135\end{array}$ & $\begin{array}{l}\text { Poisons could be lost or } \\
\text { uranium concentrated by } \\
\text { solubility. }\end{array}$ & $\begin{array}{l}\text { Container will provide } \\
\text { protection in short } \\
\text { term. }\end{array}$ & $\begin{array}{l}\text { Cannot demonstrate } \\
\text { compliance in long } \\
\text { term. }\end{array}$ \\
\hline $\begin{array}{l}\text { Consider oxidation/ } \\
\text { reduction reactions }\end{array}$ & $\begin{array}{l}10 \text { CFR60. } \\
135\end{array}$ & $\begin{array}{l}\text { Poisons could be lost or } \\
\text { uranium concentrated by } \\
\text { Redox reactions. }\end{array}$ & $\begin{array}{l}\text { Containers will provide } \\
\text { protection in short } \\
\text { term }\end{array}$ & $\begin{array}{l}\text { Cannot demonstrate } \\
\text { compliance in long } \\
\text { term. }\end{array}$ \\
\hline Consider corrosion & $\begin{array}{l}10 C F R 60 . \\
135\end{array}$ & $\begin{array}{l}\text { Poisons could be lost or } \\
\text { uranium concentrated by } \\
\text { corrosion of container } \\
\text { and fuel. }\end{array}$ & $\begin{array}{l}\text { Containers will provide } \\
\text { protection in short } \\
\text { term. }\end{array}$ & $\begin{array}{l}\text { Cannot demonstrate } \\
\text { compliance in long } \\
\text { term. }\end{array}$ \\
\hline Consider hydriding & $\begin{array}{l}10 \text { CFR60. } \\
135\end{array}$ & $\begin{array}{l}\text { May result in more } \\
\text { reactivity of fuel, } \\
\text { excessive corrosion. }\end{array}$ & $\begin{array}{l}\text { Containers will provide } \\
\text { protection in short } \\
\text { term. }\end{array}$ & $\begin{array}{l}\text { Cannot demonstrate } \\
\text { compl iance in long } \\
\text { term. }\end{array}$ \\
\hline
\end{tabular}




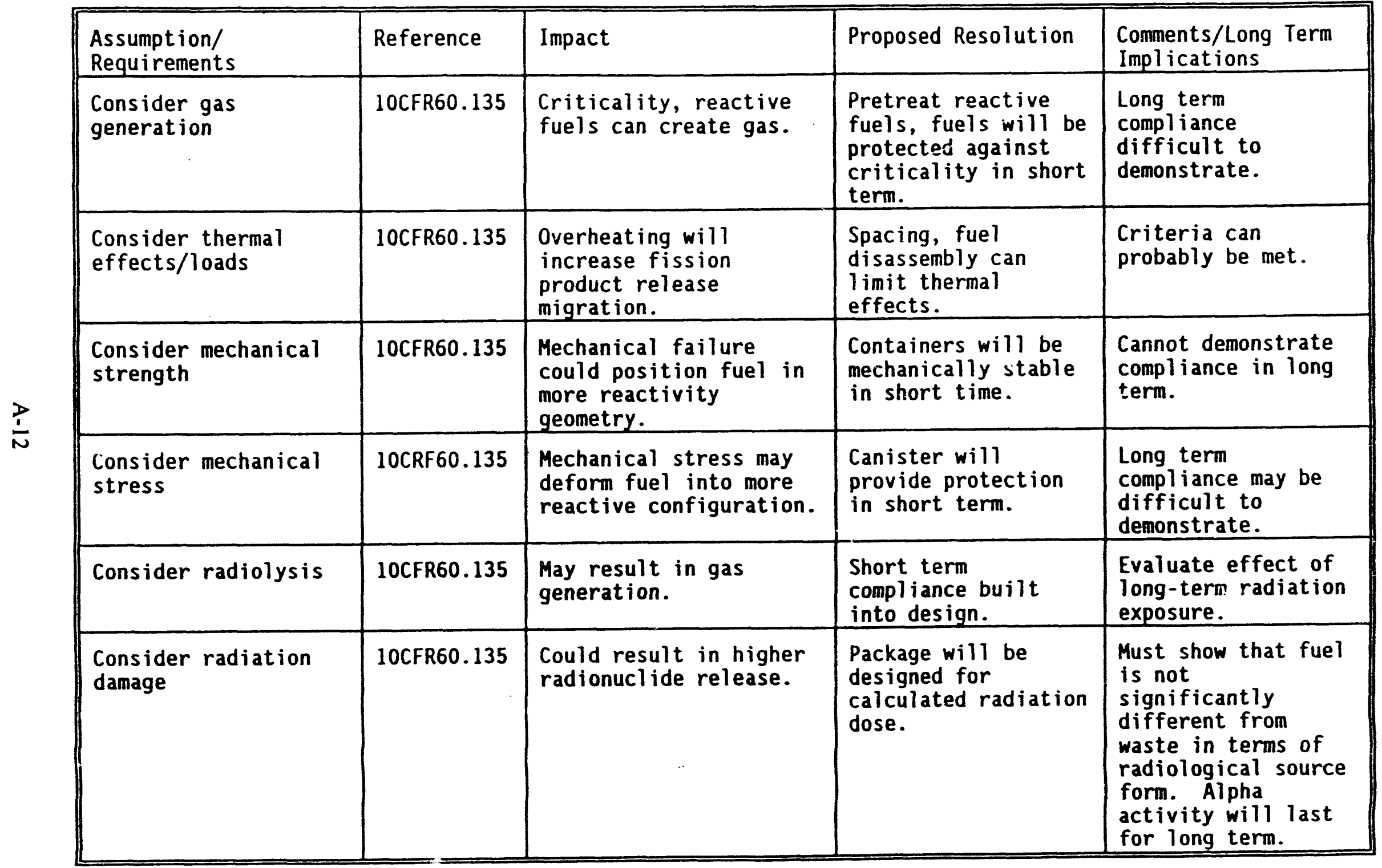




\begin{tabular}{|c|c|c|c|c|}
\hline $\begin{array}{l}\text { Assumption/ } \\
\text { Requirements }\end{array}$ & Reference & Impact & Proposed Resolution & $\begin{array}{l}\text { Comments/Long Term } \\
\text { Implications }\end{array}$ \\
\hline $\begin{array}{l}\text { Consider } \\
\text { radionuclide } \\
\text { retardation }\end{array}$ & 10CFR60.135 & $\begin{array}{l}\text { Fuel must be as good } \\
\text { as waste form for } \\
\text { fission product } \\
\text { acceleration. }\end{array}$ & $\begin{array}{l}\text { Characterize fuel to } \\
\text { demonstrate ability to } \\
\text { retain radionuclides. }\end{array}$ & $\begin{array}{l}\text { Characterization } \\
\text { will be expensive, } \\
\text { time consuming; } \\
\text { must retain } \\
\text { radionucl ides from } \\
\text { a criticality. }\end{array}$ \\
\hline Consider leaching & 10CFR60.135 & $\begin{array}{l}\text { Leaching may remove } \\
\text { poison or concentrate } \\
\text { uranium. Water will } \\
\text { increase fuel } \\
\text { reactivity. Fuel } \\
\text { must be as resistant } \\
\text { as waste form. }\end{array}$ & $\begin{array}{l}\text { Short term compliance will } \\
\text { be provided by package. }\end{array}$ & $\begin{array}{l}\text { Long-term } \\
\text { compliance will be } \\
\text { difficult to } \\
\text { demonstrate. }\end{array}$ \\
\hline $\begin{array}{l}\text { Consider fire and } \\
\text { explosion hazards }\end{array}$ & 10CFR60.135 & $\begin{array}{l}\text { Some fuels may be } \\
\text { combustible/reactive. }\end{array}$ & $\begin{array}{l}\text { Pretreat combustible/ } \\
\text { reactive fuels prior to } \\
\text { disposal. }\end{array}$ & $\begin{array}{l}\text { Pretreatment will } \\
\text { require extensive } \\
\text { facilities. }\end{array}$ \\
\hline $\begin{array}{l}\text { Consivier } \\
\text { synergistic } \\
\text { interaction }\end{array}$ & 10CRF 60.135 & $\begin{array}{l}\text { Criticality control } \\
\text { requires added } \\
\text { dimension of } \\
\text { requirements. }\end{array}$ & $\begin{array}{l}\text { Adequate plans, } \\
\text { calculations and methods } \\
\text { can be prepared. }\end{array}$ & $\begin{array}{l}\text { Increased time and } \\
\text { effort will be } \\
\text { required. }\end{array}$ \\
\hline $\begin{array}{l}\text { No pyrophoric } \\
\text { materials }\end{array}$ & $\begin{array}{l}\text { 1OCFR60.135 } \\
\text { DOE } 5480.5\end{array}$ & $\begin{array}{l}\text { Pyrophoric fuel would } \\
\text { have to be } \\
\text { pretreated. }\end{array}$ & Pretreat required fuel. & $\begin{array}{l}\text { Pretreatment } \\
\text { facility would be } \\
\text { costly. }\end{array}$ \\
\hline
\end{tabular}




\begin{tabular}{|c|c|c|c|c|}
\hline Assumption/Requirements & Reference & Impact & Proposed Resolution & $\begin{array}{l}\text { Comments/Long } \\
\text { Term } \\
\text { Implications }\end{array}$ \\
\hline $\begin{array}{l}\text { No chemically reactive } \\
\text { materials }\end{array}$ & $\begin{array}{l}10 \text { CFR60. } \\
135\end{array}$ & $\begin{array}{l}\text { Combined fuels would } \\
\text { require pretreatment. }\end{array}$ & $\begin{array}{l}\text { Pretreat fuel as } \\
\text { necessary. }\end{array}$ & $\begin{array}{l}\text { Pretreatment } \\
\text { facility would } \\
\text { be costly. }\end{array}$ \\
\hline Consolidate Particulates & $\begin{array}{l}\text { 10CFR60. } \\
135\end{array}$ & $\begin{array}{l}\text { Powdered fuel or } \\
\text { residue would have to } \\
\text { be consolidated. }\end{array}$ & $\begin{array}{l}\text { Pretreat fuel as } \\
\text { necessary. }\end{array}$ & $\begin{array}{l}\text { Pretreatment } \\
\text { facility would } \\
\text { be costly. }\end{array}$ \\
\hline $\begin{array}{l}\text { Reduce to noncombustible } \\
\text { form }\end{array}$ & $\begin{array}{l}10 \text { CFR60. } \\
135\end{array}$ & $\begin{array}{l}\text { Certain fueis would } \\
\text { require pretreatment. }\end{array}$ & $\begin{array}{l}\text { Pretreat fuels as } \\
\text { necessary. }\end{array}$ & $\begin{array}{l}\text { Pretreatment } \\
\text { would be costly. }\end{array}$ \\
\hline $\begin{array}{l}\text { Maintain dose to public } \\
\text { telow } 25 \mathrm{mr} \text { whole body }\end{array}$ & $\begin{array}{l}40 C R F 191 \\
03\end{array}$ & None for short term. & $\begin{array}{l}\text { Provide adequate } \\
\text { packaging/ shielding. }\end{array}$ & $\begin{array}{l}\text { Difficult to } \\
\text { demonstrate for } \\
\text { long term. }\end{array}$ \\
\hline $\begin{array}{l}\text { Releases to have less } \\
\text { than } 1 / 1000 \text { chance of } \\
\text { exceeding release limits }\end{array}$ & $\begin{array}{l}40 \text { CFR } 191 \\
13\end{array}$ & $\begin{array}{l}\text { Fuel must be as good } \\
\text { a form as waste. }\end{array}$ & $\begin{array}{l}\text { Characterize fuel; } \\
\text { preprocess if required }\end{array}$ & $\begin{array}{l}\text { Long-term } \\
\text { compliance } \\
\text { difficult to } \\
\text { prove. }\end{array}$ \\
\hline Monitor disposal systems & $\begin{array}{l}40 \text { CFR } 191 \\
13\end{array}$ & $\begin{array}{l}\text { Install monitoring } \\
\text { system. }\end{array}$ & $\begin{array}{l}\text { Monitoring practical in } \\
\text { short term. }\end{array}$ & $\begin{array}{l}\text { Cannot verify } \\
\text { compliance in } \\
\text { long term. }\end{array}$ \\
\hline $\begin{array}{l}\text { Do not preclude removal } \\
\text { of waste }\end{array}$ & $\begin{array}{l}40 \text { CFR } 191 . \\
14\end{array}$ & Design for removal. & $\begin{array}{l}\text { Repository/package } \\
\text { design in short term. }\end{array}$ & $\begin{array}{l}\text { Cannot verify in } \\
\text { long term. }\end{array}$ \\
\hline
\end{tabular}




\begin{tabular}{|c|c|c|c|c|}
\hline $\begin{array}{l}\text { Assumption/ } \\
\text { Requirements }\end{array}$ & Reference & Impact & Proposed Resolution & $\begin{array}{l}\text { Comments/Long Term } \\
\text { Implications }\end{array}$ \\
\hline $\begin{array}{l}\text { Consider all } \\
\text { potential. } \\
\text { criticality } \\
\text { hazards }\end{array}$ & DOE 5480.5 & $\begin{array}{l}\text { Criticality } \\
\text { prevention adds } \\
\text { additional } \\
\text { requirements and } \\
\text { care. }\end{array}$ & $\begin{array}{l}\text { Repository design, } \\
\text { fuel size. }\end{array}$ & $\begin{array}{l}\text { Cannot verify in long } \\
\text { term. }\end{array}$ \\
\hline $\begin{array}{l}\text { Ensure process is } \\
\text { subcritical under } \\
\text { both normal and } \\
\text { abnormal } \\
\text { operating/credible } \\
\text { conditions }\end{array}$ & $\begin{array}{l}\text { DOE } 5480.5 \\
\text { ANSI/ANS } \\
8.1-1983 \\
\text { ANSI/ANS } \\
8.19-1984\end{array}$ & $\begin{array}{l}\text { Extra care required } \\
\text { in handling/storage. }\end{array}$ & $\begin{array}{l}\text { Spacing, poisoning, } \\
\text { etc. may be required. } \\
\text { in package. }\end{array}$ & $\begin{array}{l}\text { Long term compliance } \\
\text { cannot be guaranteed. }\end{array}$ \\
\hline $\begin{array}{l}\text { Avoid entry of } \\
\text { water into storage } \\
\text { area }\end{array}$ & DOE 5480.5 & $\begin{array}{l}\text { Fuel reactivity will } \\
\text { be increased in wet } \\
\text { environment }\end{array}$ & $\begin{array}{l}\text { Repository } \\
\text { construction will } \\
\text { provide compliance } \\
\text { for short term. } \\
\end{array}$ & $\begin{array}{l}\text { Difficult to demonstrate } \\
\text { for long term. }\end{array}$ \\
\hline $\begin{array}{l}\text { Poisons can be } \\
\text { used if available } \\
\text { data assures } \\
\text { presence and } \\
\text { reliability }\end{array}$ & DOE 5480.5 & $\begin{array}{l}\text { Loss of poison will } \\
\text { result in increased } \\
\text { fuel reactivity. }\end{array}$ & $\begin{array}{l}\text { Container will } \\
\text { provide assurance in } \\
\text { short term but } \\
\text { cannot inspect to } \\
\text { assure presence. }\end{array}$ & $\begin{array}{l}\text { Long-term presence of } \\
\text { poison cannot be } \\
\text { demonstrated. Cannot } \\
\text { inspect to guarantee } \\
\text { presence. }\end{array}$ \\
\hline $\begin{array}{l}\text { Assure prescribed } \\
\text { extent of } \\
\text { moderation remains } \\
\text { unchanged }\end{array}$ & DOE 5480.5 & $\begin{array}{l}\text { Increased moderation } \\
\text { will increase fuel } \\
\text { reactivity. }\end{array}$ & $\begin{array}{l}\text { Container/repository } \\
\text { design will provide } \\
\text { assurance in short } \\
\text { term. }\end{array}$ & $\begin{array}{l}\text { Long term changes are } \\
\text { likely but cannot be } \\
\text { specified. }\end{array}$ \\
\hline $\begin{array}{l}\text { Reilection based } \\
\text { on result of } \\
\text { credible accident }\end{array}$ & DOE 5480.5 & $\begin{array}{l}\text { Increased reflection } \\
\text { will increase fuel } \\
\text { reactivity. }\end{array}$ & $\begin{array}{l}\text { Repository design } \\
\text { will provide } \\
\text { assurance in short } \\
\text { term. }\end{array}$ & $\begin{array}{l}\text { Long-term geological } \\
\text { events may change } \\
\text { dimensions/spacing. }\end{array}$ \\
\hline
\end{tabular}




\begin{tabular}{|c|c|c|c|c|}
\hline Assumption/Requirements & Reference & Impact & Proposed Resolution & $\begin{array}{l}\text { Comments/Long } \\
\text { Term } \\
\text { Implications } \\
\end{array}$ \\
\hline $\begin{array}{l}\text { Maintain all dimensions } \\
\text { and nuclear properties } \\
\text { relied on for geometry } \\
\text { control }\end{array}$ & DOE 5480.5 & $\begin{array}{l}\text { Change in } \\
\text { geometry may } \\
\text { increase fuel } \\
\text { reactivity. }\end{array}$ & $\begin{array}{l}\text { Original spacing can } \\
\text { be relied on for short } \\
\text { term. }\end{array}$ & $\begin{array}{l}\text { Long-term } \\
\text { geological } \\
\text { events may } \\
\text { change } \\
\text { dimensions/ } \\
\text { spacing. } \\
\end{array}$ \\
\hline $\begin{array}{l}\text { Periodic inspections, } \\
\text { insitu tests, and } \\
\text { preventive maintenance } \\
\text { needed for criticality } \\
\text { control }\end{array}$ & DOE 5480.5 & $\begin{array}{l}\text { Inspection } \\
\text { cannot easily } \\
\text { be made after } \\
\text { installation in } \\
\text { repository. }\end{array}$ & None & $\begin{array}{l}\text { Inspection, etc. } \\
\text { impossible in } \\
\text { long term. }\end{array}$ \\
\hline $\begin{array}{l}\text { Control spacing, mass, } \\
\text { density and geometry } \\
\text { under normal and } \\
\text { abnormal conditions }\end{array}$ & $\begin{array}{l}\text { ANSI/ANS-8.19 } \\
1984\end{array}$ & $\begin{array}{l}\text { Control is iost } \\
\text { after placement } \\
\text { in repository. }\end{array}$ & None & $\begin{array}{l}\text { Physicai changes } \\
\text { to repository, } \\
\text { fuels, etc. } \\
\text { possible in long } \\
\text { term. }\end{array}$ \\
\hline $\begin{array}{l}\text { Spent fuel storage } \\
\text { facility to meet } \\
\text { Category I seismic } \\
\text { requirements }\end{array}$ & RG 1.13 & $\begin{array}{l}\text { Seismic events } \\
\text { could rearrange } \\
\text { fuel, resulting } \\
\text { in criticality. }\end{array}$ & $\begin{array}{l}\text { Repository design will } \\
\text { provide protection in } \\
\text { the short term. }\end{array}$ & $\begin{array}{l}\text { Demonstration of } \\
\text { long term } \\
\text { compliance is } \\
\text { difficult. }\end{array}$ \\
\hline $\begin{array}{l}\text { Obviate concern for } \\
\text { criticality in event of } \\
\text { fire, flood, earthquake, } \\
\text { or other natural } \\
\text { calamities. }\end{array}$ & $\begin{array}{l}\text { ANS-8.7/ANSI } \\
\text { N16.5-1975 }\end{array}$ & $\begin{array}{l}\text { Natural } \\
\text { phenomena may } \\
\text { result in } \\
\text { increased fuel } \\
\text { reactivity. }\end{array}$ & $\begin{array}{l}\text { Repository design } \\
\text { should meet standard } \\
\text { in short term. }\end{array}$ & $\begin{array}{l}\text { Demonstration of } \\
\text { long term } \\
\text { compliance is } \\
\text { difficult. }\end{array}$ \\
\hline
\end{tabular}




\title{
MEs \\ Idaho Netional Engineoring Laboratory
}

\author{
Neb-04-91 \\ from R. L. Nebeker \\ Phone $6-3578 /$ MS 5219 \\ Dale October 29, 1991 \\ sublect Review of Task Team 12 Criticality Information
}

To N. A. Chipman, Fellow Engineer

New Business and Special Studies
cc:
G. G. Frandsen
V. C. Maio
R. N. Henry
B. R. Wheeler
G. W. Hogg
R. L. Nebeker - 2

Reference: R. L. Nebeker, Letter Neb-03-91, to N. A. Chipman, "Criticality Implications of Direct Disposal of Fuel," dated October 22, 1991.

At your request, the information submitted by Task Team 12 concerning criticality implications of direct fuel disposal was reviewed. In that submittal, various regulations were referenced which apply to criticality prevention. All of these references except two were described earlier in the reference to this letter. Task Team 12 referred to two NRC Regulatory Guides not included in Letter Neb-03-91: Reg Guide 3.41 and Reg Guide 3.48.

NRC Regulatory Guide 3.41 was withdrawn by the NRC on March 20, 1986 and has been replaced with NRC Regulatory Guide 3.4 (see Attachment 1). Consequently, it was not included in Neb-03-91. NRC Regulatory Guide 3.48 describes the format and contents for safety analys is reports (SARs) for fuel storage. It lists certain information to be included in the SAR which indirectly impacts criticality prevention during fuel storage. For completeness, these requirements, and implications of meeting the requirements, are shown in Attachment 2. A summary of both regulatory guides is shown in Attachment 3 in the same format as originally shown for other regulations in Neb-03-91.

If further information is needed, please let me know.

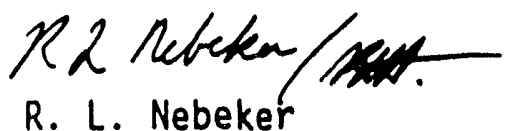

Fellow Engineer

$/ t 1$

Attachments 


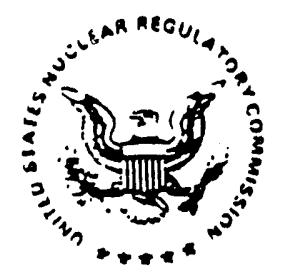

\author{
UNITED STATES \\ NUCLEAR REGULATORY COMMISSION
}

WASHINGTON. O.C. 20555

Yarch 20, 1986

\begin{abstract}
REGULATORY GUIDE OISTRIBUTION LIST (DIVISION 3)
SUBJECT: ISSUANCE OF REVISION 2 TO REGULATORY GUIDE 3.4 AND WITADRAWAL OF REGULATORY GUIDE 3.41
\end{abstract}

With the issuance of Revision 2 to Regulatory Guide 3.4, "Nuclear Criticality Safety in Operations with Fissionable Materials at Fuels and Materials Facllities," the NRC staff is withdrawing Regulatory Guide 3.4 "Validation of Calculational Methods for Nuclear Criticality Safety."

Revision 2 to Regulatory Guide 3.4 endorses ANSI/ANS-8.1-1983, "Nuclear Criticality Safety in Operations with Fissionabie Materials Outside Reactors," which is a consolidation of ANSI N16.1-1975/ANS-8.1 (endorser by Revision 1 of Regulatory Guide 3.4) and ANSI N16.9-1975/ANS-8.11 iendorsed by Regulatory Guide 3.41). Regulatory Guide 3.41 is therefor: obsolete. However, withdrawal of Regulatory Guide 3.41 is in no way intended to alter any prior or existing licensing commitments based on its use.

Regulatory guides may be withdrawn when they are superseded by the Commission's regulations, when equivalent recommendations have been incorpcrated in applicable approved codes and standards, or when change: in methods and techniques or in the need for specific guidance have mad: then obsolete.

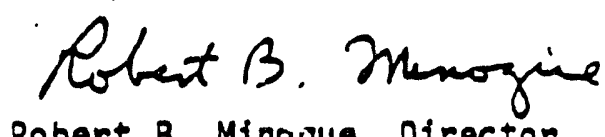

Robert B. Minogue, Director Office of Nuclear Regulatory Researci 
Attachment 2

Criticality and Other Requirements for Spent Fuel Disposal

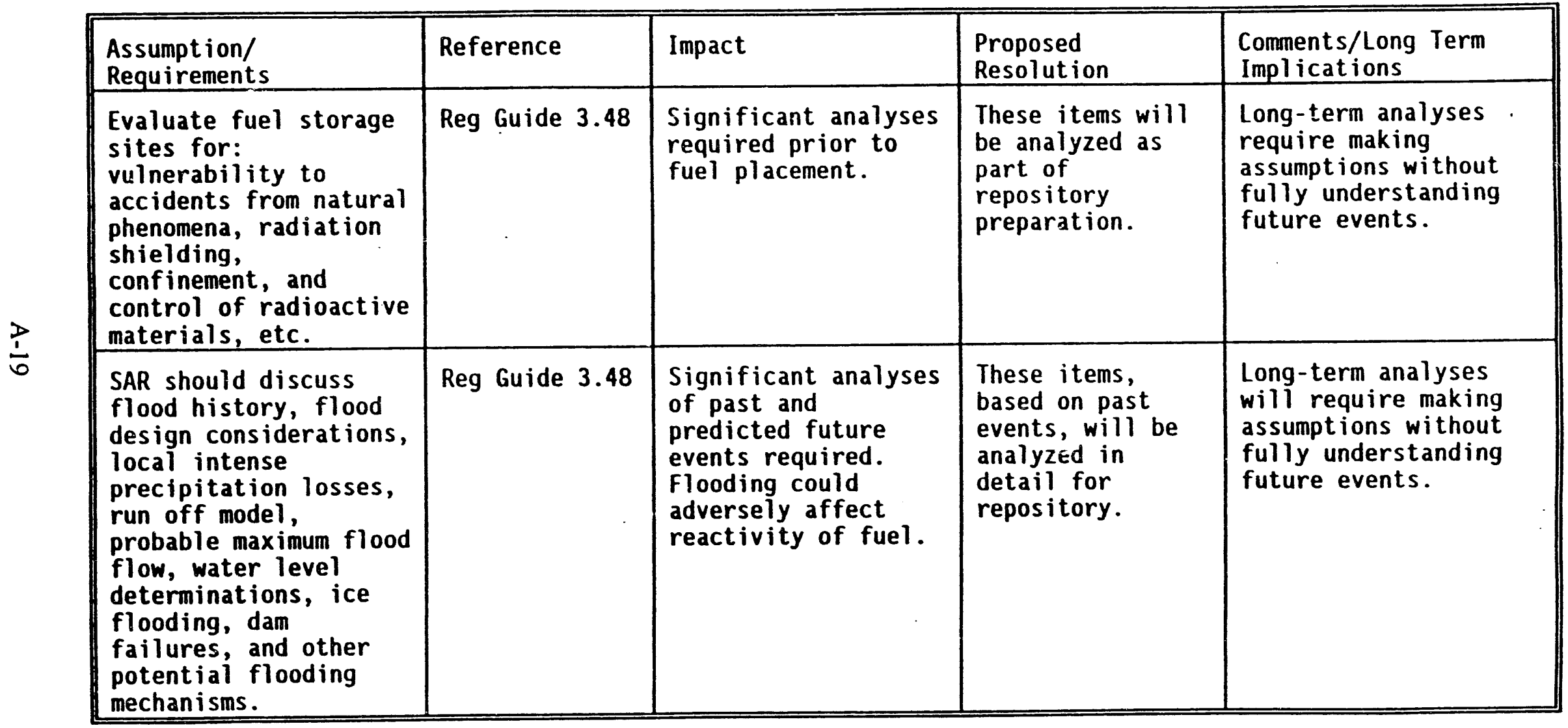




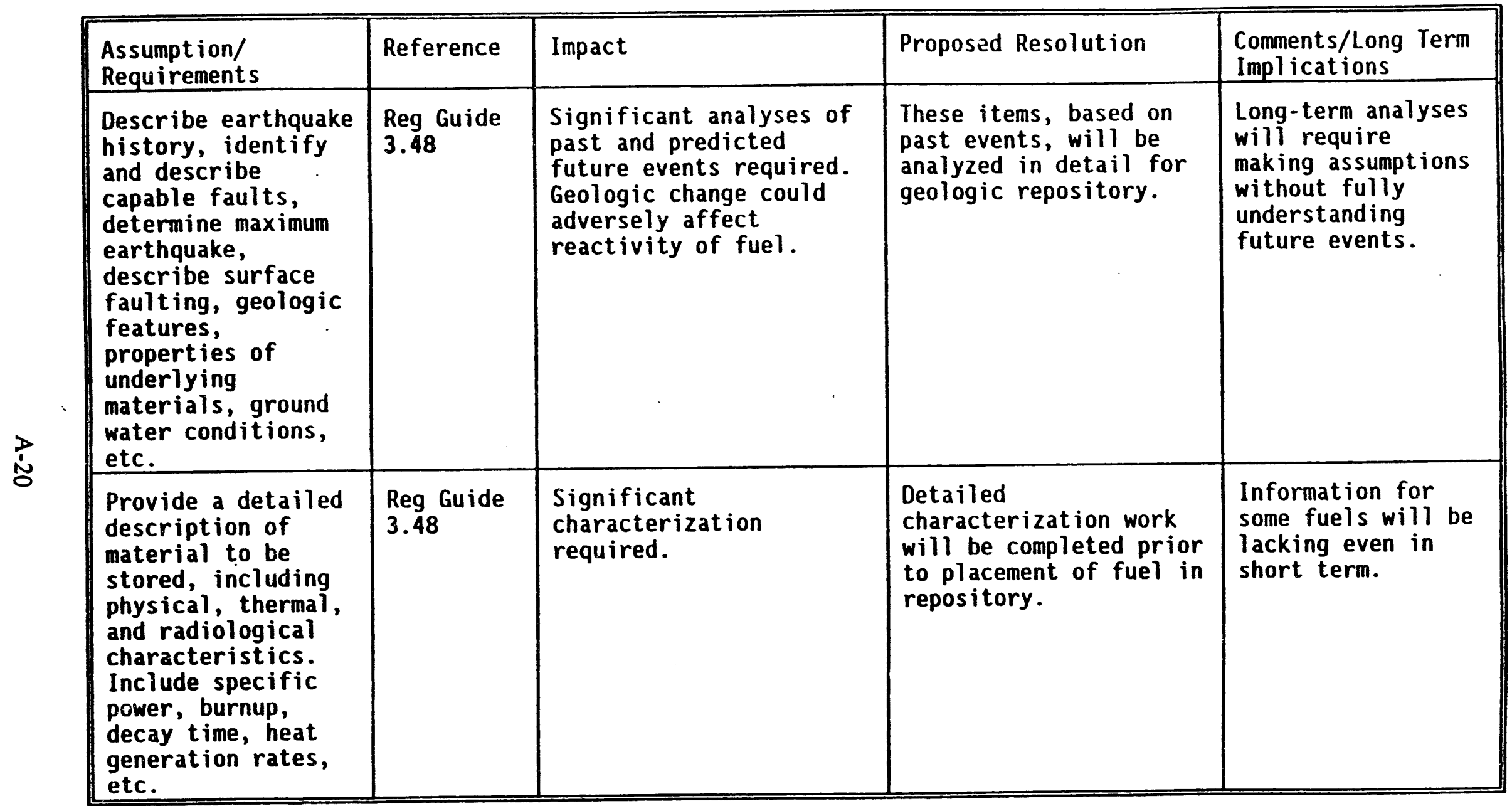




\begin{tabular}{|c|c|c|c|c|}
\hline $\begin{array}{l}\text { Provide } \\
\text { information } \\
\text { concerning tornado } \\
\text { missiles, floods, } \\
\text { flood elevations, } \\
\text { flood force, flood } \\
\text { protection, } \\
\text { seismic design, } \\
\text { snow and ice } \\
\text { loadings, etc. }\end{array}$ & $\begin{array}{l}\text { Reg Guide } \\
3.48\end{array}$ & $\begin{array}{l}\text { Significant analyses } \\
\text { required. Flooding } \\
\text { or fuel movement } \\
\text { could adversely } \\
\text { affect fuel } \\
\text { reactivity. }\end{array}$ & $\begin{array}{l}\text { Analyses will be completed } \\
\text { at time of repository } \\
\text { construction. }\end{array}$ & $\begin{array}{l}\text { Long-term analyses } \\
\text { will require } \\
\text { making assumptions } \\
\text { without fully } \\
\text { understanding } \\
\text { future events. }\end{array}$ \\
\hline $\begin{array}{l}\text { Describe control } \\
\text { methods for } \\
\text { prevention of } \\
\text { criticality and } \\
\text { error contingency } \\
\text { criteria. } \\
\text { Describe the } \\
\text { design features, } \\
\text { procedures, and } \\
\text { special techniques } \\
\text { to preclude } \\
\text { criticality. }\end{array}$ & $\begin{array}{l}\text { Reg Guide } \\
3.48\end{array}$ & $\begin{array}{l}\text { Significant analyses } \\
\text { required. }\end{array}$ & $\begin{array}{l}\text { Analyses will be completed } \\
\text { at time of repository } \\
\text { construction. }\end{array}$ & $\begin{array}{l}\text { Certain control } \\
\text { methods cannot be } \\
\text { guaranteed to be } \\
\text { viable for long } \\
\text { terms. }\end{array}$ \\
\hline $\begin{array}{l}\text { Describe systems } \\
\text { for providing } \\
\text { safety under } \\
\text { normal and } \\
\text { abnormal } \\
\text { conditions. }\end{array}$ & $\begin{array}{l}\text { Reg Guide } \\
3.48\end{array}$ & $\begin{array}{l}\text { Extensive } \\
\text { descriptions will be } \\
\text { required. }\end{array}$ & $\begin{array}{l}\text { Description will be } \\
\text { required when repository } \\
\text { is built. }\end{array}$ & $\begin{array}{l}\text { Facility and } \\
\text { system will not } \\
\text { remain in original } \\
\text { configuration for } \\
\text { long periods. }\end{array}$ \\
\hline
\end{tabular}




\begin{tabular}{|l|l|l|l|l|}
\hline Assumption/Requirements & Reference & Impact & Proposed Resolution & $\begin{array}{l}\text { Comments/Long } \\
\text { Term } \\
\text { Implications }\end{array}$ \\
\hline $\begin{array}{l}\text { Analyze potential } \\
\text { accidents, including } \\
\text { causes, detection, } \\
\text { effects and } \\
\text { consequences, corrective } \\
\text { actions, etc. }\end{array}$ & $\begin{array}{l}\text { Reg Guide } \\
3.48\end{array}$ & $\begin{array}{l}\text { Extensive analyses } \\
\text { will be required. }\end{array}$ & $\begin{array}{l}\text { Analyses will be part } \\
\text { of geologic repository } \\
\text { SAR. }\end{array}$ & $\begin{array}{l}\text { Long-term } \\
\text { analyses must be } \\
\text { based on } \\
\text { inadequate } \\
\text { information. }\end{array}$ \\
\hline $\begin{array}{l}\text { Conduct of Operation } \\
\text { items to be described. }\end{array}$ & $\begin{array}{l}\text { Reg Guide } \\
3.48\end{array}$ & $\begin{array}{l}\text { Descriptions will be } \\
\text { prepared. }\end{array}$ & $\begin{array}{l}\text { These conditions will } \\
\text { be followed during } \\
\text { storage of fuel. }\end{array}$ & $\begin{array}{l}\text { These conditions } \\
\text { cannot be } \\
\text { guaranteed for } \\
\text { long time } \\
\text { periods. }\end{array}$ \\
\hline
\end{tabular}


Attachment 3

Summary of Guidance Documents Pertaining to Criticality and Fuel Storage

\begin{tabular}{|l|l|l||}
\hline Document & Title & Summary of Contents \\
\hline $\begin{array}{l}\text { NRC } \\
\text { Regulatory } \\
\text { Guide 3.41 }\end{array}$ & $\begin{array}{l}\text { Validation of } \\
\text { calculational methods for } \\
\text { nuclear criticality } \\
\text { safety. }\end{array}$ & Withdrawn 3/20/86 and replaced by Regulatory Guide 3.4. \\
\hline $\begin{array}{l}\text { NRC } \\
\text { Regulatory } \\
\text { Guide 3.48. }\end{array}$ & $\begin{array}{l}\text { Standard format and } \\
\text { content for the Safety } \\
\text { Analysis Report for an } \\
\text { independent spent fuel } \\
\text { storage installation or } \\
\text { monitored retrievable } \\
\text { storage installation (dry } \\
\text { storage). }\end{array}$ & $\begin{array}{l}\text { This guide presents a standard format that is acceptable to } \\
\text { the NRC staff for the SAR required for a license } \\
\text { application to store spent fuel in an independent spent } \\
\text { high-level radioactive waste in a monitored retrievable } \\
\text { storage installation. }\end{array}$ \\
\hline \multirow{2}{*}{ storage installation or to store spent fuel and }
\end{tabular}



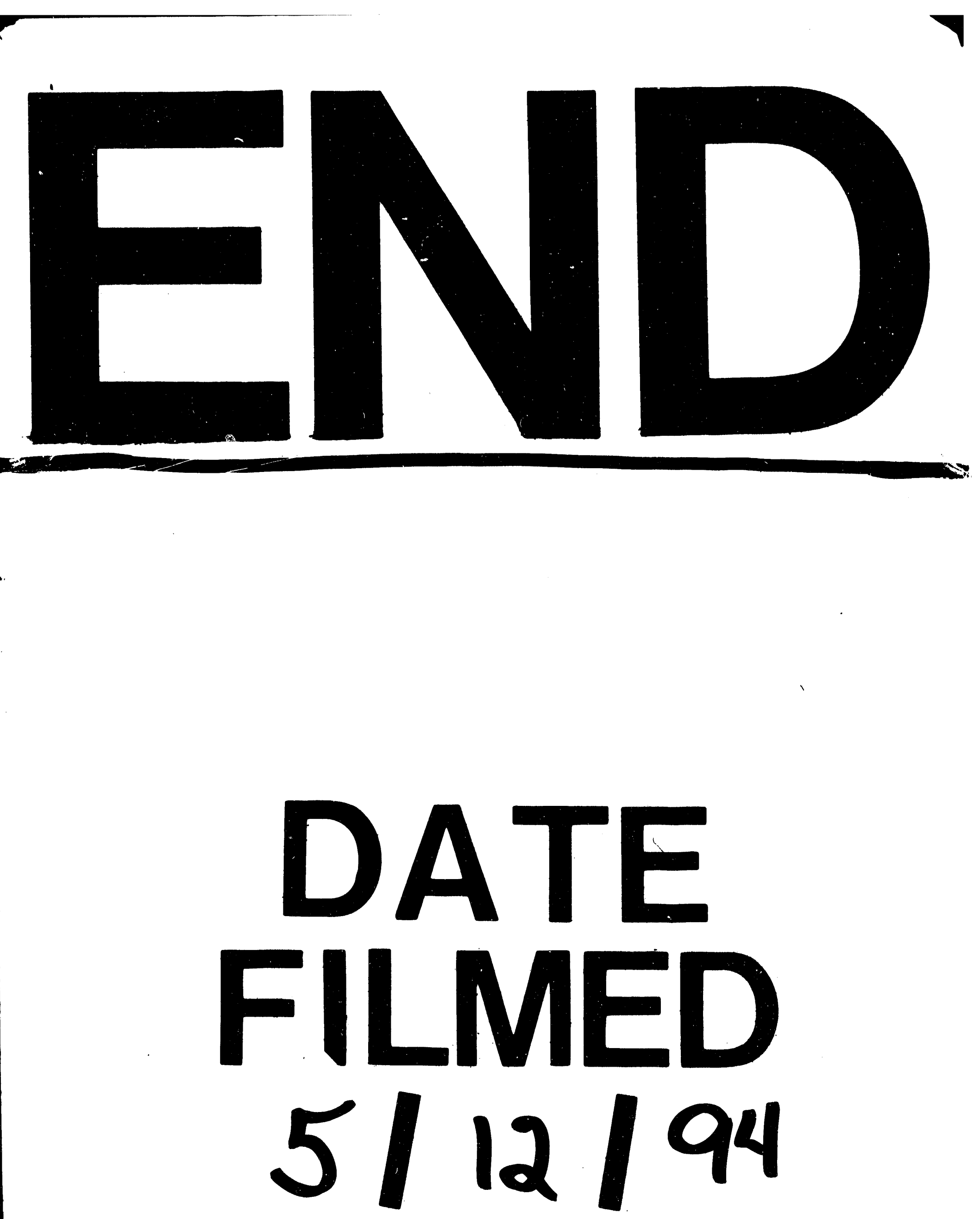
